\title{
Multiple physical elements to determine the gravitational-wave signatures of core-collapse supernovae
}

\author{
Kei Kotake ${ }^{\mathrm{a}, \mathrm{b}}$ \\ ${ }^{a}$ Division of Theoretical Astronomy, National Astronomical Observatory of Japan, 2-21-1, Osawa, Mitaka, Tokyo, \\ 181-8588, Japan \\ ${ }^{b}$ Center for Computational Astrophysics, National Astronomical Observatory of Japan, Mitaka, Tokyo 181-8588, Japan
}

\begin{abstract}
We review recent progress in the theoretical predictions of gravitational waves (GWs) of corecollapse supernovae. Following a brief summary of the methods in the numerical modeling, we summarize multiple physical elements that determine the GW signatures which have been considered to be important in extracting the information of the long-veiled explosion mechanism from the observation of the GWs. We conclude with a summary of the most urgent tasks to make the dream come true.
\end{abstract}

Keywords: Supernovae, Radiation-Hydrodynamics, Gravitational Wave Physics, Neutrino Physics

PACS number:97.60Bw,95.30.Lz,97.60Jd,95.85Sz,97.60.-s, 04.30.-w,04.40.Dg

\section{Introduction}

Massive stars in the approximate mass range of 8 to 100 solar masses $\left(M_{\odot}\right)$ end their lives as core-collapse supernovae. They have long attracted the attention of astrophysicists because they have many aspects playing important roles in astrophysics. They are the mother of neutron stars and black holes; they play an important role for acceleration of cosmic rays; they influence galactic dynamics triggering further star formation; they are gigantic emitters of neutrinos and gravitational waves. They are also a major site for nucleosynthesis, so, naturally, any attempt to address human origins may need to start with an understanding of core-collapse supernovae $(\mathrm{SNe})$.

Current estimates of core-collapse SN rates in our Galaxy predict one event every $\sim 40 \pm 10$ year [1]. When a massive star undergoes a core-collapse SN in our Galactic center, copious numbers of neutrinos are produced, some of which may be detected on the earth. Such "supernova neutrinos" will carry valuable information from deep inside the core. In fact, the detection of neutrinos from SN1987A (albeit in the Large Magellanic Cloud) opened up the neutrino astronomy, which is an alternative to conventional astronomy by electromagnetic waves [2, 3]. Even though there were just two dozen neutrino events from SN1987A, these events have been studied extensively (yielding $\sim 500$ papers) and have allowed us to have a confidence that our basic picture of the supernova physics is correct (e.g., [4], see [5] for a recent review). If a supernova occurs in our Galactic center $(\sim 10 \mathrm{kpc})$, about 10,000 $\bar{v}_{e}$ events are estimated to be detected by Super-Kamiokande (SK) [6] (e.g., [7, 8]). Those successful neutrino detections are important 
not only to study the supernova physics but also to unveil the nature of neutrinos itself such as the neutrino oscillation parameters and the mass hierarchy (e.g., [9] for a recent review).

Core-collapse SNe are now about to start even another astronomy, Gravitational-Wave Astronomy. Currently long-baseline laser interferometers such as LIGO (USA) [10], VIRGO (Italy) 1 , GEO600 (Germany), and TAMA300 (Japan) [11] are currently operational and preparing for the first observation (see, e.g., [12] for a recent review), by which the prediction by Einstein's theory of General Relativity (GR) can be confirmed. These instruments are being updated to their Advanced status, and may start taking data, possibly detecting GWs for the first time, as soon as 2015 (see [13] for a recent review). In fact, Advanced LIGO/VIRGO, which is an upgrade of the initial LIGO and VIGRO, are expected to be completed by 2015 and will increase the observable detection volume by a factor of $\sim 1000$ [14]. The Large-scale Cryogenic Gravitational-wave Telescope (LCGT [15]) in Japan was funded in late 2010, which is being built under the Kamioka mine and is expected to take its first data in 2016. At such a high level of precision, those GW detectors are sensitive to many different sources, including chirp, ring-down, and merger phases of black-hole and neutron star binaries (e.g., [16, 17, 18]), neutron star normal mode oscillations (e.g., [19]), rotating neutron star mountains (e.g., [20]), and core-collapse supernova explosions (e.g., [21, 22, 23] for recent reviews), on the final of which we focus in this article.

According to the Einstein's theory of general relativity (e.g., [24]), no GWs can be emitted if gravitational collapse of the supernova core proceeds perfectly spherically symmetric. To produce GWs, the gravitational collapse should proceed aspherically and dynamically. Observational evidence gathered over the last few decades has pointed towards core-collapse SNe indeed being generally aspherical (e.g., [25, 26, 27, 28] and references therein). The most unequivocal example is SN1987A. The HST images of SN1987A are directly showing that the expanding envelope is elliptical with the long axis aligned with the rotation axis inferred from the ring ([26], see however [29] for a recent counter argument). The aspect ratio and position angle of the symmetry axis are consistent with those predicted earlier from the observations of speckle and linear polarization. What is more, the linear polarization became greater as time passed (e.g., $[25,30,31]$ ), a fact which has been used to argue that the central engine of the explosion is responsible for the non-sphericity (e.g., [32, 33] ). From a theoretical point of view, clarifying what makes the dynamics of the core deviate from spherical symmetry is essential in understanding the GW emission mechanism. Here it is worth mentioning that GWs are primary observables, which imprint a live information of the central engine, because they carry the information directly to us without being affected in propagating from the stellar center to the earth. On the other hand, SN neutrinos, which are affected by the well-known Mikheyev-Smirnov-Wolfenstein (MSW) effect (e.g., [34]) in propagating the stellar envelope, may be rather indirect in extracting the information of the central engine compared to GWs 3

The breaking of the sphericity in the supernova engine has been also considered as a most important ingredient to understand the explosion mechanism, for which supernova theorists have been continuously keeping their efforts for the past $\sim 40$ years. Albeit being a topic of hot debate yet, current multi-dimensional (multi-D) simulations based on refined numerical models show several promising scenarios. Among the candidates are the neutrino heating mechanism aided by convection and hydrodynamic instabilities of the supernova shock (e.g., [36] for a review),

\footnotetext{
${ }^{1}$ http://www.ego-gw.it/

${ }^{2}$ http://geo600.aei.mpg.de/

${ }^{3}$ However they can be a useful tool like a tomography to monitor the density profile in the stellar mantle, which is expected to tell us the evolution of the supernova shock (e.g., [35] for a recent review).
} 
the acoustic mechanism [37], or the magnetohydrodynamic (MHD) mechanism (e.g., [21] see references therein).

Putting things together, the multi-dimensionality determines the explosion mechanism, in turn we may extract the information that traces the multi-dimensionality by the SN messengers, which would be only possible by a careful analysis primary on GWs as well as neutrinos, and photons. In this review article, we hope to bring together findings from recent multi-D supernova simulations and the obtained predictions of the GWs so far (for GWs from other high-energy astrophysical sources such as magnetars, gamma-ray bursts, and coalescing binaries, see [38, 39, 40, 41] for recent reviews). Before moving on to the next sections, we first have to draw a caution that the current generation of numerical results that we report in this article and the associated predictions of the GW emission should depend on the next-generation calculations by which more sophistication can be made not only in determining the efficiency of neutrinomatter coupling (the so-called neutrino transport problem), but also in the treatment of general relativity. Therefore we provide here only a snapshot of the moving (long-run) documentary film that records our endeavours for making our dream of "GW astronomy of core-collapse supernovae" come true.

\section{On core-collapse supernova simulations}

In order to extract a true astrophysical GW signal from the confusing detector noises, we need not only require sensitive detectors, but also extensive data analysis of the detector output based on reliable theoretical estimates for the GW signals. In most cases, a detailed numerical modeling is required to make the precise prediction of the dynamics for the GW sources. As will be summarized briefly in this section, $\mathrm{SN}$ modelers have been keeping their efforts to this end for these +45 years.

The abstract in the paper by Colgate and White in 1966 [42] who reported the first SN simulation finished with the sentence,"The energy release (at the moment of explosion) corresponds to the change in gravitational potential of the unstable imploding core; the transfer of energy takes place by the emission and deposition of neutrinos' 4 . As well-known, this is the essence of the so-called neutrino-heating mechanism of core-collapse supernovae. The mechanism was first proposed to occur in a prompt manner, but was later reinforced by Bethe and Wilson [43, 44, 45] to take a currently prevailing delayed form, in which a stalled bounce shock is revived by neutrino energy deposition to trigger explosions in several hundred milliseconds after bounce. The mechanism has been the working hypothesis of supernova theorists for these $\sim 45$ years (see collective references in Janka et al. (2007) [36] that was presented in the Bethe Centennial Volume in Physics Report, and also a summary given in section 2 of Nordhaus et al. (2010) [46]).

However, one important lesson we have learned from a series of the most up-to-date simulations [47, 48, 49, 50] which implemented the best input physics and numerics to date, is that the mechanism fails to blow up canonical massive star:5 in spherical symmetric (1D) simulations. Pushed by supernova observations of the blast morphology [e.g., 25, 28] mentioned above, it is now almost certain that the breaking of the spherical symmetry is the key to solve the supernova problem. So far a number of multi-D hydrodynamic simulations have been reported, which demonstrated that hydrodynamic motions associated with convective overturn

\footnotetext{
${ }^{4}$ I boldly added several words in (...) for making the meaning of the sentence complete.

${ }^{5}$ except for $8-9 M_{\odot}$ stars (e.g., [51]).
} 
(e.g., [52, 53, 54, 55, 56]) as well as the Standing-Accretion-Shock-Instability (SASI, e.g., [57, 58, 59, 60, 61, 62, 63, 64, 65, 66, 67, 68] see references therein) can help the onset of the neutrino-driven explosion.

To test the neutrino heating mechanism in the multi-D context, it is of crucial importance to solve accurately the neutrino-matter coupling in spatially non-uniform hydrodynamic environments. For the purpose, one ultimately needs to solve the six-dimensional (6D) neutrino radiation transport problem (three in space, three in the momentum space of neutrinos), thus the supernova simulation has been counted as one of the most challenging tasks in numerical astrophysics. In the final sentence of the last paragraph, we wrote "demonstrated" because the neutrino heating was given by hand as an input parameter in most of the simulations cited above (see, however [55, 56]). The neutrino heating proceeds dominantly via the charged current interactions $\left(v_{\mathrm{e}}+\mathrm{n} \rightleftarrows \mathrm{e}^{-}+\mathrm{p}, \bar{v}_{\mathrm{e}}+\mathrm{p} \rightleftarrows \mathrm{e}^{+}+\mathrm{n}\right)$ in the gain region. The neutrino heating rate in the gain region can be roughly expressed as [69] $Q^{+} \propto L_{v}\left\langle\mu_{v}\right\rangle^{-1} / r^{2}$ where $L_{v}$ is the neutrino luminosity emitted from the surface of neutrino sphere and it determines the amplitude of the neutrino heating as well as cooling, and $r$ and $\left\langle\mu_{v}\right\rangle$ is the distance from the stellar center and the flux factort, respectively (e.g., [69]). For example, $L_{v}$ is treated as an input parameter in the so-called "light-bulb" approach (e.g., [54]). This is one of the most prevailing approximations in recent $3 \mathrm{D}$ simulations $[66,67,70,46]$ because it is handy to study multi-D effects on the neutrino heating mechanism (albeit on the qualitative grounds). To go beyond the light-bulb scheme, $L_{v}$ should be determined in a self-consistent manner. For the purpose, one needs to tackle with neutrino transport problem, only by which energy as well as angle dependence of the neutrino distribution function can be determined without any assumptions. Since the focus of this review is on GWs, a detailed discussion of various approximations and numerical techniques taken in the recent radiation-hydrodynamic SN simulations cannot be provided. Table 1 is not intended as a comprehensive compilation, but we just want to summarize milestones that have recently reported exploding models so far. The table will be useful in later sections when we discuss the GW signatures obtained in some of these simulations.

In Table 1,the first column ("Progenitor") shows the progenitor model employed in each simulation. The abbreviation of "NH", "WHW", and "WW" means Nomoto \& Hashimoto (1988) [71], Woosley, Heger, and Weaver (2002) [72], and Woosley and Weaver (1995) [73]. The second column shows SN groups with the published or submitted year of the corresponding work. "MPA" stands for the core-collapse SN group in Max Planck Institute for Astrophysics led by H.T. Janka and E. Müller. "Princeton+" stands for the group chiefly consisting of the staffs in the Princeton University (A. Burrows), Caltech (C.D.Ott), Hebrew University (E. Livne) and their collaborators. The SN group in the Basel university is led by M. Liebendörfer and F.K. Thielemann. "OakRidge+" stands for the SN group mainly consisting of the Florida Atlantic University (S. Bruenn) and the Oak Ridge National Laboratory (A. Mezzacappa, O.E.B. Messer) and their collaborators. NAOJ+ is the SN group chiefly consisting of the staffs in the National Astronomical Observatory of Japan (myself, T. Takiwaki), Kyoto university (Y. Suwa), Waseda University (S. Yamada), and their collaborators. The third column represents the mechanism of explosions which are basically categorized into two (to date), namely by the neutrino-heating mechanism (indicated by " $v$-driven") or by the acoustic mechanism ("Acoustic"). "Dim." in the fourth column is the fluid space dimensions which is one-, two-, or three-dimension $(1,2,3 \mathrm{D})$. The abbreviation "N" stands for 'Newtonian,' while "PN"—-for 'Post-Newtonian' — stands for

\footnotetext{
${ }^{6}$ This quantity represents the degree of anisotropy in neutrino emission; $\left\langle\mu_{v}\right\rangle \sim 0.25$ near at the neutrino sphere, $\left\langle\mu_{v}\right\rangle=1$ in the free-streaming limit $(r \rightarrow \infty)$ (e.g., Janka (2001) 69]).
} 
some attempt at inclusion of general relativistic effects, and "GR" denotes full relativity. $t_{\exp }$ in the fifth column indicates an approximate typical timescale when the explosion initiates and $E_{\exp }$ represents the explosion energy normalized by Bethe $\left(=10^{51} \mathrm{erg}\right)$ given at the postbounce time of $t_{\mathrm{pb}}$, both of which are attempted to be sought in literatures 7 . In the final column of " $v$ transport", "Dim" represents $v$ momentum dimensions and the treatment of the velocity dependent term in the transport equations is symbolized by $O(v / c)$. The definition of the "RBR", "IDSA", and "MGFLD" will be given soon in the following.

Due to the page limit of this article, we have to start the story only after 2006 (see, e.g., Janka et al. (2007) [36] for a grand review, and also Cardall (2005) [83] for a similar table before 2006). The first news of the exploding model was reported by the MPA group. By using their MuDBaTH code which includes one of the best available neutrino transfer approximations, they reported 1D and 2D explosions for the $8.8 M_{\odot}$ star by NH88 whose progenitor has a very tenuous outer envelope with steep density gradient, which is a characteristic property of AGB stars. Also in $1 \mathrm{D}$, the Basel+ group reported explosions for 10 and $15 M_{\odot}$ progenitors of WHW02 triggered by the hypothesised first-order QCD phase transition in the protoneutron star (PNS). To date, these two are the only modern numerical results where the neutrino-driven mechanism succeeded in 1D. In the 2D MPA simulations, they obtained explosions for a non-rotating 11.2 $M_{\odot}$ progenitor of WHW02 [77], and then for a $15 M_{\odot}$ progenitor [82] of WW95 with a relatively rapid rotation imposed 8 . They newly brought in the so-called "ray-by-ray" approach (indicated by "RBR" in the table), in which the neutrino transport is solved along a given radial direction assuming that the hydrodynamic medium for the direction is spherically symmetric. This method, which reduces the 2D problem partly to $1 \mathrm{D}$, fits well with their original 1D Boltzmann solver 4779. For 2D hydrodynamic simulations with the ray-by-ray transport, one needs to solve the 4D radiation transport problem (two in space and two in the neutrino momentum space). Regarding the explosion energies obtained in the MPA simulations, their values at their final simulation time are typically underpowered by one or two orders of magnitudes to explain the canonical supernova kinetic energy ( $\left.\sim 10^{51} \mathrm{erg}\right)$. But the explosion energies presented in their figures are still growing with time, and they could be as high as $1 \mathrm{~B}$ if they were able to follow a much longer evolution as discussed in [77].

More recently, fully 2D multi-angle Boltzmann transport simulations become practicable by the Princeton+ group [86, 87]. In this case, one needs to handle the 5D problem for 2D simulations (two in space, and three in the neutrino momentum space). However this scheme is very computationally expensive currently to perform long-term supernova simulations. In fact, the most recent 2D work by [87] succeeded in following the dynamics until $400 \mathrm{~ms}$ after bounce for a non-rotating and a rapidly rotating $20 M_{\odot}$ model of WHW02, but explosions seemingly have not been obtained in such an earlier phase either by the neutrino-heating or the acoustic mechanism.

In the table, "MGFLD" stands for the Multi-Group Flux-Limited Diffusion scheme which eliminates the angular dependence of the neutrino distribution function (see, e.g., Bruenn (1985) [88] for more details). For 2D simulations, one needs to solve the 3D problem, namely two in space, and one in the neutrino momentum space. By implementing the MGFLD algorithm to the

\footnotetext{
${ }^{7}$ but if we cannot find them, we remain them as blank ".".

${ }^{8}$ by comparing the precollapse angular velocity to the one predicted in a recent stellar evolution calculation [84].

${ }^{9}$ Note that the ray-by-ray approach has an advantage compared to other approximation schemes, such that it can fully take into account the available neutrino reactions (e.g., [85] for references therein) and also give us the most accurate solution for a given angular direction.
} 


\begin{tabular}{|c|c|c|c|c|c|c|}
\hline Progenitor & $\begin{array}{l}\text { Group } \\
\text { (Year) }\end{array}$ & Mechanism & $\begin{array}{l}\text { Dim. } \\
\text { (Hydro) }\end{array}$ & $\begin{array}{l}t_{\exp } \\
(\mathrm{ms})\end{array}$ & $\begin{array}{c}E_{\exp }(\mathrm{B}) \\
\text { @ } t_{\mathrm{pb}}(\mathrm{ms})\end{array}$ & $\begin{array}{c}v \text { transport } \\
(\mathrm{Dim}, O(v / c))\end{array}$ \\
\hline \multirow{2}{*}{$\begin{array}{l}8.8 M_{\odot} \\
(\mathrm{NH} 88[71])\end{array}$} & $\begin{array}{l}\text { MPA }[51,74] \\
(2006)\end{array}$ & $v$-driven & $\begin{array}{c}1 \mathrm{D} / 2 \mathrm{D} \\
(\mathrm{PN})\end{array}$ & $\sim 200$ & $\begin{array}{c}0.1 \\
(\sim 800)\end{array}$ & $\begin{array}{c}\text { Boltzmann } \\
2, O(v / c)\end{array}$ \\
\hline & $\begin{array}{l}\text { Princeton+ } \\
{[75](2006)}\end{array}$ & $v$-driven & $\begin{array}{l}2 \mathrm{D} \\
(\mathrm{N})\end{array}$ & $\lesssim 125$ & $\begin{array}{c}0.1 \\
-\end{array}$ & $\begin{array}{c}\text { MGFLD } \\
1,(\mathrm{~N})\end{array}$ \\
\hline $\begin{array}{l}10 M_{\odot} \\
(\text { WHW02[72]) }\end{array}$ & $\begin{array}{l}\text { Basel[76] } \\
(2009)\end{array}$ & $\begin{array}{c}v+(\mathrm{QCD} \\
\text { transition })\end{array}$ & $\begin{array}{l}1 \mathrm{D} \\
\text { (GR) }\end{array}$ & 255 & $\begin{array}{c}0.44 \\
(350)\end{array}$ & $\begin{array}{c}\text { Boltzmann } \\
2,(\mathrm{GR})\end{array}$ \\
\hline $\begin{array}{l}11 M_{\odot} \\
\text { (WW95[73] }\end{array}$ & $\begin{array}{l}\text { Princeton+ } \\
\text { [75](2006) }\end{array}$ & Acoustic & $\begin{array}{l}2 \mathrm{D} \\
(\mathrm{N})\end{array}$ & $\gtrsim 550$ & $\begin{array}{l}\sim 0.1^{*} \\
(1000)\end{array}$ & $\begin{array}{c}\text { MGFLD } \\
1,(\mathrm{~N})\end{array}$ \\
\hline \multirow{3}{*}{$\begin{array}{l}11.2 M_{\odot} \\
(\mathrm{WHW} 02[72])\end{array}$} & $\begin{array}{l}\text { MPA[77] } \\
(2006)\end{array}$ & $v$-driven & $\begin{array}{l}2 \mathrm{D} \\
(\mathrm{PN})\end{array}$ & $\sim 100$ & $\begin{array}{l}\sim 0.005 \\
(\sim 220)\end{array}$ & $\begin{array}{l}\text { "RBR" Boltz- } \\
\text { mann, } 2, O(v / c)\end{array}$ \\
\hline & $\begin{array}{l}\text { Princeton+ } \\
\text { [78] (2007) }\end{array}$ & Acoustic & $\begin{array}{l}2 \mathrm{D} \\
(\mathrm{N})\end{array}$ & $\gtrsim 1100$ & $\begin{array}{l}\sim 0.1^{*} \\
(1000)\end{array}$ & $\begin{array}{c}\text { MGFLD } \\
1,(\mathrm{~N})\end{array}$ \\
\hline & $\begin{array}{c}\text { NAOJ+ } \\
\text { [79](2011) }\end{array}$ & $v$-driven & $\begin{array}{l}\text { 3D } \\
(\mathrm{N})\end{array}$ & $\sim 100$ & $\begin{array}{l}0.01 \\
(300)\end{array}$ & $\begin{array}{l}\text { IDSA } \\
1,(\mathrm{~N})\end{array}$ \\
\hline $\begin{array}{l}12 M_{\odot} \\
\text { (WHW02[72]) }\end{array}$ & $\begin{array}{l}\text { Oak Ridge+ } \\
{[80](2009)}\end{array}$ & $v$-driven & $\begin{array}{l}2 \mathrm{D} \\
(\mathrm{PN})\end{array}$ & $\sim 300$ & $\begin{array}{c}0.3 \\
(1000)\end{array}$ & $\begin{array}{c}\text { "RBR" MGFLD } \\
1, O(v / c)\end{array}$ \\
\hline $\begin{array}{l}13 M_{\odot} \\
\text { (WHW02[72]) }\end{array}$ & $\begin{array}{l}\text { Princeton+ } \\
{[78](2007)}\end{array}$ & Acoustic & $\begin{array}{l}2 \mathrm{D} \\
(\mathrm{N})\end{array}$ & $\gtrsim 1100$ & $\begin{array}{l}\sim 0.3^{*} \\
(1400)\end{array}$ & $\begin{array}{c}\text { MGFLD } \\
1,(\mathrm{~N})\end{array}$ \\
\hline (NH88[71]) & $\begin{array}{c}\text { NAOJ+ } \\
{[81](2010)}\end{array}$ & $v$-driven & $\begin{array}{l}2 \mathrm{D} \\
(\mathrm{N})\end{array}$ & $\sim 200$ & $\begin{array}{c}0.1 \\
(500)\end{array}$ & $\begin{array}{l}\text { IDSA } \\
1,(N)\end{array}$ \\
\hline $\begin{array}{l}15 M_{\odot} \\
\text { (WW95[73]) }\end{array}$ & $\begin{array}{l}\text { MPA[82] } \\
(2009)\end{array}$ & $v$-driven & $\begin{array}{l}2 \mathrm{D} \\
(\mathrm{PN})\end{array}$ & $\sim 600$ & $\begin{array}{l}0.025 \\
(\sim 700)\end{array}$ & $\begin{array}{c}\text { Boltzmann } \\
2, O(v / c)\end{array}$ \\
\hline \multirow[t]{2}{*}{ (WHW02[72]) } & $\begin{array}{c}\text { Princeton+ } \\
\text { [78] }\end{array}$ & Acoustic & $\begin{array}{l}2 \mathrm{D} \\
(\mathrm{N})\end{array}$ & - & $\begin{array}{l}- \\
(-)\end{array}$ & $\begin{array}{c}\text { MGFLD } \\
1,(\mathrm{~N})\end{array}$ \\
\hline & $\begin{array}{l}\text { OakRidge+ } \\
\text { [80](2009) }\end{array}$ & $v$-driven & $\begin{array}{l}2 \mathrm{D} \\
(\mathrm{PN})\end{array}$ & $\sim 300$ & $\begin{array}{l}\sim 0.3 \\
(600)\end{array}$ & $\begin{array}{c}\text { "RBR" MGFLD } \\
1, O(v / c)\end{array}$ \\
\hline $\begin{array}{l}20 M_{\odot} \\
\text { (WHW02[72]) }\end{array}$ & $\begin{array}{l}\text { Princeton+ } \\
{[78](2007)}\end{array}$ & Acoustic & $\begin{array}{l}2 \mathrm{D} \\
(\mathrm{N})\end{array}$ & $\gtrsim 1200$ & $\begin{array}{l}\sim 0.7^{*} \\
(1400)\end{array}$ & $\begin{array}{l}\text { MGFLD } \\
1,(\mathrm{~N})\end{array}$ \\
\hline \multirow[t]{2}{*}{$\begin{array}{l}25 M_{\odot} \\
\text { (WHW02[72]) }\end{array}$} & $\begin{array}{l}\text { Princeton+ } \\
\text { [78](2007) }\end{array}$ & Acoustic & $\begin{array}{l}2 \mathrm{D} \\
(\mathrm{N})\end{array}$ & $\gtrsim 1200$ & $\begin{array}{l}- \\
(-)\end{array}$ & $\begin{array}{l}\text { MGFLD } \\
1,(\mathrm{~N})\end{array}$ \\
\hline & $\begin{array}{l}\text { Oak Ridge+ } \\
\text { [80](2009) }\end{array}$ & $v$-driven & $\begin{array}{l}2 \mathrm{D} \\
(\mathrm{PN})\end{array}$ & $\sim 300$ & $\begin{array}{c}\sim 0.7 \\
(1200)\end{array}$ & $\begin{array}{c}\text { "RBR" MGFLD } \\
1, O(v / c)\end{array}$ \\
\hline
\end{tabular}

Table 1: Selected lists of recent neutrino-radiation hydrodynamic milestones reported by many SN groups around the world ("Group"), which obtained explosions by the neutrino-heating mechanism (indicated by " $v$-driven") or the acoustic mechanism ("Acoustic") (See text for more details). 
CHIMERA code in a ray-by-ray fashion (e.g., [80]), Bruenn et al. (2009) [89] obtained neutrinodriven explosions for non-rotating progenitors in a relatively wide range in $12,15,20,25 M_{\odot}$ of WHW02 (see table). These models tend to start exploding at around $300 \mathrm{~m}$ after bounce, and the explosion energy for the longest running model of the $25 \mathrm{M}_{\odot}$ progenitor is reaching to $1 \mathrm{~B}$ at 1.2 $\mathrm{s}$ after bounce [80].

On the other hand, the 2D MGFLD simulations implemented in the VULCAN code [75] obtained explosions for a variety of progenitors of $11,11.2,13,15,20$, and $25 M_{\odot}$ not by the neutrino-heating mechanism but by the acoustic mechanism 10 . The acoustic mechanism relies on the revival of the stalled bounce shock by the energy deposition via the acoustic waves that the oscillating protoneutron stars (PNSs) would emit in a much delayed phase ( 1 second) compared to the conventional neutrino-heating mechanism ( 300-600 milliseconds). If the core pulsation energy given in Burrows et al. (2007) [78] could be used to measure the explosion energy in the acoustic mechanism, they reach to $1 \mathrm{~B}$ after $1000 \mathrm{~ms}$ after bounce 11 . The additional energy input from acoustic waves is very appealing, but it may remain a matter of vivid debate and has yet to be confirmed by other groups.

By performing $2 \mathrm{D}$ simulations in which the spectral neutrino transport was solved by the isotropic diffusion source approximation (IDSA) scheme [90], the NAOJ+ group reported explosions for a non-rotating and rapidly rotating $13 M_{\odot}$ progenitor of NH88. They pointed out that a stronger explosion is obtained for the rotating model comparing to the corresponding nonrotating model. The IDSA scheme splits the neutrino distribution into two components (namely the streaming and trapped neutrinos), both of which are solved using separate numerical techniques (see, Liebendörfer et al. (2009) [90] for more details). The approximation level of the IDSA scheme is basically the same as the one of the MGFLD. The main advantage of the IDSA scheme is that the fluxes in the transparent region can be determined by the non-local distribution of sources rather than the gradient of the local intensity like in MGFLD. A drawback in the current version of the IDSA scheme is that heavy lepton neutrinos $\left(v_{x}\right.$, i.e., $v_{\mu}, v_{\tau}$ and their anti-particles) as well as the energy-coupling weak interactions have yet to be implemented. Extending the 2D modules in Suwa et al. (2009) [81] to 3D, they recently reported explosions in the 3D models for an 11.2 $M_{\odot}$ progenitor of WHW02 (Takiwaki et al.(2011) [79]). By comparing the convective motions as well as neutrino luminosities and energies between their $2 \mathrm{D}$ and 3D models, they pointed out whether 3D effects would help explosions or not is sensitive to the employed numerical resolutions. They argued that next-generation supercomputers are at least needed to draw a robust conclusion of the $3 \mathrm{D}$ effects.

Having summarized a status of the current supernova simulations, one might easily see a number of issues that remain to be clarified. First of all, the employed progenitors usually rather scatter (e.g., Table 1). Different SN groups seem to have a tendency to employ different progenitors, providing different results. By climbing over a wall which may have rather separated exchanges among the groups, a detailed comparison for a given progenitor needs to be done seriously in the multi-D results (as have been conducted in the Boltzmann 1D simulations between the MPA, Basel+, and Oak Ridge+ groups [91]).

In addition to the importance of 3D modeling as mentioned above, a more complete "realistic" supernova model should naturally include general relativity (GR) with magnetohydrodynamics (MHD) with multi-D GR Boltzmann neutrino transport, in which a microphysical treatment

\footnotetext{
${ }^{10}$ For the $8.8 M_{\odot}$ progenitor, they obtained neutrino-driven explosions (see table 1 ).

${ }^{11}$ Due to the ambiguity of the relation between the pulsation energy and the explosion energy, an asterisk is added in the table.
} 
of equation of state (EOS) and nuclear-neutrino interactions are appropriately implemented. Unfortunately none of the currently published SN simulations satisfy the "ultimate" requirement. In this sense, all the mentioned studies employ some approximations (albeit with different levels of sophistication) for multiple physical ingredients (for example, as listed in Table 112).

In the same way, theoretical predictions of the GWs that one can obtain by analyzing the currently available numerical results, cannot unambiguously give us the final answer yet. Again we hereby note the feature of this article which shows only a snapshot of the moving theoretical terrain. Keeping this caveat in mind, it is also true that a number of surprising GW features of core-collapse SNe have been reported recently both by the first-principle simulations (e.g., in Table 1), and also by idealized simulations in which explosions are parametrically initiated mostly by the light-bulb scheme. As will be mentioned in the next section, the latter approach is also useful to get a better physical understanding of the GW signatures obtained in the firstprinciple simulations. We are now ready to move on to focus on the GW signatures from the next section.

\section{GW signatures}

We first start to present a short overview concerning possible emission cites of GWs in corecollapse SNe so far proposed by a number of extensive studies (section 3.0.1, see also [21, 22, 23] for recent reviews). The most up-to-date results regarding the GW signatures emitted near bounce in the case of rapidly rotating core-collapse, have been already given in the recent review by Ott (2009) (e.g., section 4 in [22]). So in this article, we mainly focus on the GWs in the postbounce phase in the context of neutrino-driven explosion models and also on the postbounce GWs expected in MHD explosions, which will be described separately in sections 3.1 and 3.2.

\subsubsection{A short overview}

The paper in 1982 by E. Müller of the MPA entitled as "Gravitational Radiation from Collapsing Rotating Stellar Cores" unquestionably opened our eyes to the importance of making the GW prediction based on realistic SN numerical modeling (see e.g., section 2 in [22] for a summary of more earlier work which had mainly focused on the GW emission in very idealized systems such as in homogeneous spheroids and ellipsoids 14 . As one may expect from the title of his paper, rapid rotation, if it would exist in the precollapse iron core, leads to significant rotational flattening of the collapsing and bouncing core, which produces a time-dependent quadrupole (or higher) GW emission. Following the first study by Müller, most studies of the past thirty have focused on the so-called bounce signals (e.g., [98, 99, 100, 101, 102, 103, 104, $105,106,107,108,109,110,111,112$ ) and references therein).

As summarized by Ott (2009) [22], a number of important progresses have been recently made to understand features of the bounce signals by extensive 2D GR studies using the conformalflat-condition (CFC) approximation [108, 109, 110] and also by fully GR 3D simulations [105] both including realistic EOSs and a deleptonization effect based on 1D-Boltzmann simulations [113]. Due to the page limit of this article, we are only able to touch on them in section 3.2 .

\footnotetext{
${ }^{12}$ However it is also worth mentioning that a rush of nice work to this end has been reported recently including new schemes towards the GR radiation-hydrodynamic simulations [92, 93, 94] and new sets of supernova EOSs [95, 96, 97].

${ }^{13}$ In this sense, these two approaches are complimentary in understanding the GW signatures.

${ }^{14}$ Needless to say, this kind of approach is still very important to extract the physics of the GW emission mechanism.
} 
For the bounce signals having a strong and characteristic signature, the iron core must rotate enough rapidly. Although the role of rapid rotation in combination with magnetic fields is attracting great attention as an important key in understanding the dynamics of collapsars and magnetars (e.g., section 3.2), recent stellar evolution calculations predict that such a extreme condition can be realized only in a special case 11415 ( $\sim 1 \%$ of massive star population). In addition, the precollapse rotation periods are estimated to be larger than $\sim 100 \mathrm{sec}$ [116] to explain the observed rotation periods of radio pulsars. In such a slowly rotating case, the detection of the bounce signals becomes very hard even by next-generation detectors for a supernova in our Galaxy ( 10 kpc, e.g., [103, 105]).

Besides the rapid rotation, anisotropic matter motions associated with convection and anisotropic neutrino emission in the postbounce phase are expected to be primary GW sources with comparable amplitudes to the bounce signals. Thus far, various physical ingredients have been studied for producing the post-bounce asphericities, including postshock convection 117], pre-collapse density inhomogeneities [118, 117, 119, 56], moderate rotation of the iron core [120], nonaxisymmetric rotational instabilities [121, 122], g-modes [123] and r-modes pulsations [124] of PNSs, and more recently by the Standing-Accretion-Shock Instability (SASI, [125, 126, 127, 128, 129]). In the multi-D modeling of stellar evolution, significant progresses have been recently made (e.g., [130] and references therein), however the degree of initial inhomogeneities in the iron core seems still uncertain. Studies of non-radial instabilities in PNSs and cold NSs with their emission processes of GWs have a long history (e.g., section 7 in [22] for references therein). The numerical studies to this end generally treat the microphysics in a very phenomenological manner (like by a polytrope EOS), so there may remain a further room for sophistication. Among the candidate GW emission mechanisms, we therefore choose to focus on relatively well understood parts at first in the next section (section 3.1), which is the GW produced by convection and anisotropic neutrino emission.

\subsection{Gravitational waveforms in neutrino-driven explosions}

As mentioned, the first-principle and experimental simulations have a complimentary role to understand the GWs in the context of neutrino-driven SN explosions. Since the latter approach is equivalently useful, we firstly summarize recent findings obtained by the experimental simulations in the next sections 3.1.1 and 3.1.2 then proceed to the first-principle results in section 3.1.3 For the matter GW 16 signals, we review the results by Murphy et al. [129] who conducted a systematic study by changing neutrino luminosity and progenitor models. For the neutrino GW signals, a piece of our work [126] is summarized in which a ray-tracing analysis was performed to accurately estimate the neutrino GWs.

\subsubsection{Features of Matter $G W$ s in experimental $2 D$ simulations}

Figure 1 shows samples of gravitational waveforms obtained in 2D models by Murphy et al. [129]. In their 2D models, the dynamics of a suite of progenitor models $(12,15,20$, and 40 $M_{\odot}$ ) is followed starting from gravitational collapse, through bounce, up to parametric explosions via the light-bulb scheme. In each panel of Figure 1 the taken progenitor and the input (electron-)neutrino luminosity in unit of $10^{52} \mathrm{erg} / \mathrm{s}$ are indicated. For example, top left panel

\footnotetext{
${ }^{15}$ which experiences the so-called chemically homogeneous evolution [115].

${ }^{16}$ Note that "Matter GW" means the GW produced by quadrupole matter deformation and that "neutrino GW" by anisotropic neutrino emission.
} 

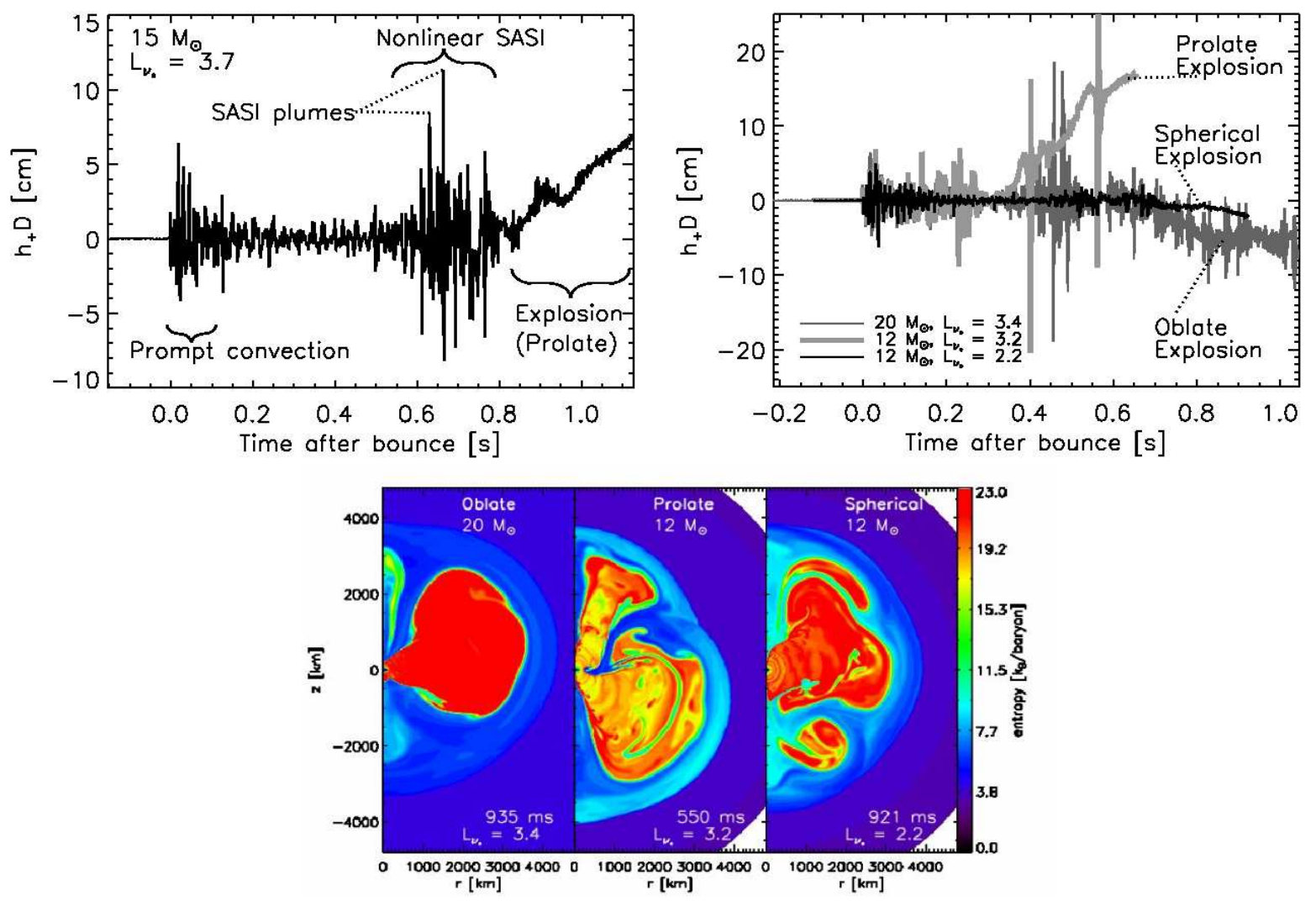

Figure 1: Samples of gravitational waveforms in 2D parametric explosion models (by courtesy of Murphy and the coauthors [129]). Top left panel shows matter GWs obtained for a $15 M_{\odot}$ progenitor model [72] with an input neutrino luminosity of $L_{v_{e}}=3.7 \times 10^{52} \mathrm{erg} / \mathrm{s}$. Three typical GW emission cites are clearly indicated, which are "Prompt convection (after bounce up to $50 \mathrm{~ms}$ after bounce )", "Postshock convection" (then till $550 \mathrm{~ms}$ after bounce, unless the word is shown in the plot), "Non-linear SASI with SASI plumes"(then till $\sim 800 \mathrm{~ms}$ after bounce), and "Explosion (then afterwards)". The top right panel shows that the GW amplitudes monotonically increase or decease with time in the "Explosion" phase reflecting the geometry of the expanding shock (bottom panel), which is either prolate (increase), oblate (decrease), or spherical (in-between). In each panel, the chosen progenitor models of [72] are given in each panel by $12,20 M_{\odot}$ and the input luminosity is indicated by the value of $L_{v_{e}}$ in unit of $10^{52} \mathrm{erg} / \mathrm{s}$. 
shows matter GWs obtained for a $15 M_{\odot}$ progenitor model [72] with neutrino luminosity of $L_{v_{e}}=3.7 \times 10^{52} \mathrm{erg} / \mathrm{s}$. Three typical GW emission cites are clearly presented, which are "Prompt convection", "Postshock convection" (unless it is shown in the plot), "Non-linear SASI with SASI plumes", and "Explosion".

Shortly after the bounce shock is formed, negative entropy gradient behind the stalling shock predominantly gives rise to prompt convection. GWs indicated by "Prompt convection" (top left panel in Figure 10 comes from this. Later on, the PNS convection driven by the negative lepton gradient near its surface and the neutrino-driven convection in the postshock heating region develop. This corresponds to the GW emission by "Postshock convection" (unless shown in the plot) after the prompt convection till $550 \mathrm{~ms}$ after bounce. Subsequently, the GW amplitudes become much more larger as the SASI enters to a non-linear phase with violent sloshing of the postshock material. Large spikes appearing in the "Non-linear SASI" phase come from the down-flowing "SASI plumes" striking the PNS surface. Afterward when the sloshing shock turns to be explosion (indicated by "Explosion"), the sign of the GW amplitudes change reflecting the geometry of the expanding shock, that is either prolate (increase), oblate (decrease), or spherical (in-between) as shown in the top right and bottom panels in Figure 1. On top of these illuminating findings, they argued that the characteristic GW frequency has a tight correlation with a deceleration timescale of the accreting material at the lower boundary of the postshock convective region. As will be mentioned in section 3.1.4 this trend is also observed in a recent 3D simulation by Müller et al. [131].

\subsubsection{Features of Neutrino GWs in experimental $2 D$ simulations}

To understand the behavior of neutrino GW $\mathrm{s}^{17}$ in $2 \mathrm{D}$ simulations, it is useful to look the stress formula. In $2 \mathrm{D}$ simulations, the nonvanishing component of the neutrino GWs can be expressed as 117

$$
h_{v}^{\mathrm{TT}}=\frac{4 G}{c^{4} R} \int_{0}^{t} d t^{\prime} \int_{0}^{\pi} d \theta^{\prime} \Phi\left(\theta^{\prime}\right) \frac{d l_{v}\left(\theta^{\prime}, t^{\prime}\right)}{d \Omega^{\prime}},
$$

where $G$ is the gravitational constant, $c$ is the speed of light, $R$ is the distance of the source to the observer, $d l_{v} / d \Omega$ represents the direction-dependent neutrino luminosity emitted per unit of solid angle into direction of $\Omega$, and $\Phi\left(\theta^{\prime}\right)$ denotes the quantity, which depends on the angle measured from the symmetry axis $\left(\theta^{\prime}\right)$,

$$
\Phi\left(\theta^{\prime}\right)=\pi \sin \theta^{\prime}\left(-1+2\left|\cos \theta^{\prime}\right|\right) .
$$

It is easy to show that the neutrino $\mathrm{GW}$ is zero if the neutrino emission is isotropic $\left(d l_{v} /\left(\theta^{\prime}, t^{\prime}\right)=\right.$ constant $)$. This function has positive values in the north polar cap for $0 \leq \theta^{\prime} \leq 60^{\circ}$ and in the south polar cap for $120^{\circ} \leq \theta^{\prime} \leq 180^{\circ}$, but becomes negative values between $60^{\circ}<\theta^{\prime}<120^{\circ}$. Therefore, the polar and equatorial excess in the neutrino emission tends to make a positive and negative change in the neutrino GWs, respectively.

Figure 2 shows properties of neutrino GWs obtained in the parametric 2D simulations by Kotake et al. (2009) [126] who used the light-bulb scheme to obtain explosions. In their simulations, the initial conditions were derived from a steady-state approximation of the postshock

\footnotetext{
${ }^{17}$ As anisotropic matter motions generate GWs, anisotropic neutrino emission also gives rise to GWs, which has been originally pointed out in late 1970's by [132, 133] (see recent progress in [134]). It is expected as a primary GW source also in gamma-ray bursts [135, 136] and Pop III stars [137].
} 

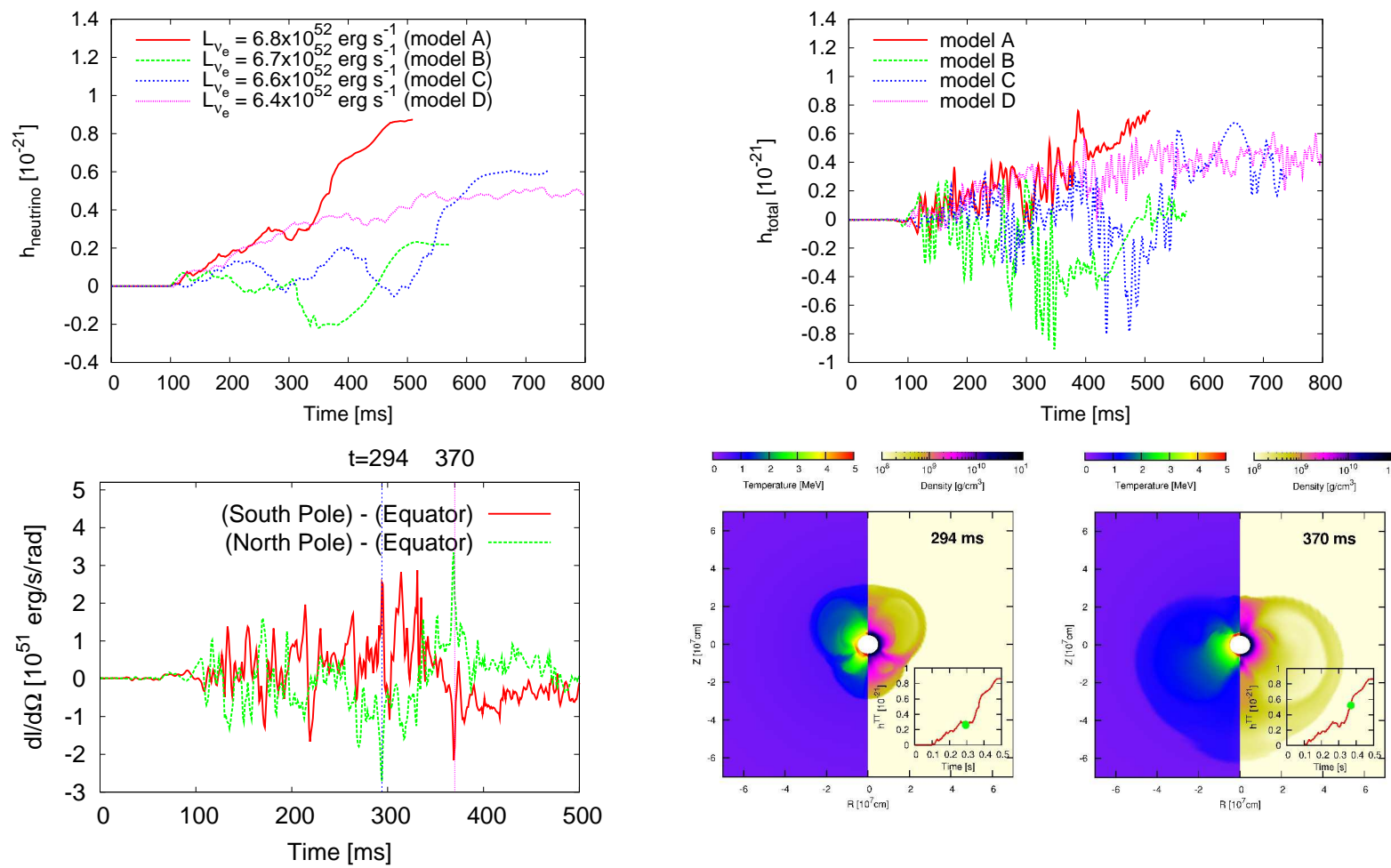

Figure 2: Gravitational waveforms obtained in 2D parametric explosion models in Kotake et al. (2009) [126] (for a distance of $10 \mathrm{kpc}$ ). For the four models (of A, B, C, and D) with different input luminosities (see text for more details), the top panels show the GWs only from neutrinos (top left) and from the sum of neutrinos and matter (top right). The time is measured from the epoch when the simulation starts from the initial condition (see text). In all the models, the SASI enters to the non-linear regime at about $100 \mathrm{~ms}$, simultaneously making the amplitudes deviate from zero. The bottom left panel shows the directional dependent neutrino luminosity: $d l_{v} / d \Omega$ for model A, in which the polar excess from equator in the luminosity is indicated by "(South pole) - (Equator)" or "(North pole) - (Equator)". Vertical lines represent the epochs of $t=294$ and $370 \mathrm{~ms}$, respectively (see text for details). For the chosen two epochs, the bottom right panel shows temperature- (the left-half of each panel) and density- (the right-half) distributions in the meridian section. The insert of each panel shows the neutrino GW amplitudes, in which the green point indicates the time of the snapshot. The central region colored by white ( $50 \mathrm{~km}$ in radius) represents the inner boundary taken in the $2 \mathrm{D}$ simulation. 
structure (not from stellar evolution calculations) and the dynamics only outside an inner boundary at $50 \mathrm{~km}$ was solved. To estimate the neutrino anisotropy (e.g., $d l_{v} /\left(\theta^{\prime}, t^{\prime}\right)$ in Equation 1), a ray-tracing analysis was performed. In the two top panels of Figure 2 the input electron neutrino luminosity $\left(L_{v_{e}}\right)$ is indicated for four models A, B, C, and D, respectively corresponding to $L_{v_{e}}=6.4,6.6,6.7$, and $6.8 \times 10^{52} \mathrm{erg} / \mathrm{s}$.

The top left panel shows the GW amplitudes contributed only from neutrinos. Comparing the right panel, which shows the total amplitudes (neutrino + matter), it can be seen that the overall structures of the waveforms are predominantly determined by the neutrino GWs with slower temporal variations ( $\gtrsim 50 \mathrm{~ms}$ ), to which the GWs from matter motions with rapid temporal variations $(\lesssim 10 \mathrm{~ms})$ are superimposed.

The bottom left panel shows the angle dependent neutrino luminosity (e.g., $d l_{v} / d \Omega$ in Equation (10) for model A, in which the polar excess from equator in the luminosity is indicated by "(South pole) - (Equator)" or "(North pole) - (Equator)". As shown, the dominance of the neutrino emission in the north (green line) and south poles (red line) are closely anti-correlated. This is the consequence of the low-mode nature of the SASI, here of $\ell=1$ (in terms of an expansion in spherical harmonics with order $\ell$ ). In fact, the left-hand side in the bottom right panel shows that at $294 \mathrm{~ms}$, the blob encompassing the regions inside the stalled shock is moving from the southern to the northern hemisphere, leading to the compression of the matter in the south hemisphere, which is vice versa at $370 \mathrm{~ms}$ (right-hand side). Recalling again that the function (Figure 2 ) is positive near poles, the polar excess in the neutrino GWs makes the positively growing feature depicted in the neutrino GWs (e.g., top left panel in Figure 2). In addition, the waveforms show large negative growth for some epochs during the growth of SASI (e.g., for models C and $\mathrm{D}$ in the top left panel). Such a feature comes from a dominance of the neutrino emission in the equatorial direction 18 . Reflecting the nature of the SASI and convection both of which grow chaotically with time, they pointed out that there may be no systematic dependence of the input neutrino luminosities on the maximum GW amplitudes.

\subsubsection{GW signatures in $2 D$ radiation-hydrodynamic simulations}

Having summarized the findings in parametric 2D simulations, we are now ready to discuss GW signatures obtained in 2D radiation-hydrodynamic simulations in which a spectral neutrino transport is solved. Similar to Table 1 , but Table 2 shows a summary for some selected GW predictions obtained in the first-principle 2D model 19 . Since the model predictions are not too many (as of 2011), the important GW features obtained mostly by the MPA simulation and also by the OakRidge+ simulation are all summarized in this section by reproducing their results as much as possible.

The first example is for a rotating $15 M_{\odot}$ progenitor model by the MPA simulation (e.g., [77], see the numerical details in Table 1), the detailed GW analysis of which was performed by Müller et al. (2004) (Figure 3). The initial angular velocity for the model was taken to be $0.5 \mathrm{rad} / \mathrm{s}$, which is slightly faster (a factor 5) than the one predicted in a recent stellar evolution calculation [84]. As shown in the top left panel, they found a quasi-monotonically increasing GW signal at the end of their simulation $(\sim 270 \mathrm{~ms}$ after bounce), which predominantly comes from neutrino GWs. In the case of rotational core-collapse, neutrino emission can be stronger along polar directions [138, 139, 140]. As discussed in section 3.1.2, the polar excess in neutrino

\footnotetext{
${ }^{18}$ To capture this trend, the ray-tracing calculation would be more accurate than a ray-by-ray approach.

${ }^{19}$ Note that much more stronger GW emission than those in Table 2 was expected if the acoustic mechanism would work. See section 7.1 in [22] for more details.
} 


\begin{tabular}{|c|c|c|c|c|c|c|}
\hline $\begin{array}{l}\text { Progenitor } \\
\text { (rotation) }\end{array}$ & $\begin{array}{l}\text { Group } \\
\text { (Year) }\end{array}$ & $\begin{array}{c}\text { Mechanism } \\
\text { (Domain) }\end{array}$ & $\begin{array}{c}\text { Dim. } \\
\text { (Hydro) }\end{array}$ & $\begin{array}{c}t_{\text {fin }} \\
(\mathrm{ms})\end{array}$ & $\begin{array}{c}\left|h_{\max }\right|(\mathrm{cm}) \\
\text { (from) }\end{array}$ & $\begin{array}{l}f_{\text {peak }} \\
(\mathrm{Hz})\end{array}$ \\
\hline $\begin{array}{l}11.2 M_{\odot}[72] \\
(\mathrm{No})\end{array}$ & $\begin{array}{c}\text { MPA[77] } \\
(2004)\end{array}$ & $\begin{array}{c}v \text {-driven } \\
\left(180^{\circ} \text { wedge }\right)\end{array}$ & $\begin{array}{c}2 \mathrm{D} \\
(\mathrm{PN})\end{array}$ & $\sim 200$ & $\begin{array}{c}\sim 30 \\
\text { (matter) }\end{array}$ & $\begin{array}{l}\sim 700 \\
\end{array}$ \\
\hline $\begin{array}{l}12 M_{\odot}[72] \\
(\mathrm{No})\end{array}$ & $\begin{array}{c}\text { Oak Ridge+ } \\
{[80](2009)}\end{array}$ & $\begin{array}{c}v \text {-driven } \\
\left(180^{\circ} \text { wedge }\right)\end{array}$ & $\begin{array}{c}2 \mathrm{D} \\
(\mathrm{PN})\end{array}$ & $\sim 550$ & $\begin{array}{c}80 \\
\text { (neutrino) }\end{array}$ & $\sim 900$ \\
\hline $\begin{array}{l}13 M_{\odot}[71] \\
\text { (Both) }\end{array}$ & $\begin{array}{c}\text { NAOJ+ } \\
{[81](2011)}\end{array}$ & $\begin{array}{c}v \text {-driven } \\
\left(180^{\circ} \text { wedge }\right)\end{array}$ & $\begin{array}{l}2 \mathrm{D} \\
(\mathrm{N})\end{array}$ & $\sim 700$ & $\begin{array}{c}18 \\
\text { (neutrino) }\end{array}$ & $\sim 600$ \\
\hline $\begin{array}{l}15 M_{\odot}[73] \\
(\mathrm{Yes})\end{array}$ & $\begin{array}{l}\mathrm{MPA}[77] \\
(2004)\end{array}$ & $\begin{array}{c}v \text {-driven } \\
\left(90^{\circ} \text { wedge }\right)\end{array}$ & $\begin{array}{c}2 \mathrm{D} \\
(\mathrm{PN})\end{array}$ & $\sim 270$ & $\begin{array}{c}\sim 150 \\
\text { (neutrino) }\end{array}$ & $\sim 600$ \\
\hline (No) & $\begin{array}{l}\text { MPA[77] } \\
(2006)\end{array}$ & $\begin{array}{c}v \text {-driven } \\
\left(180^{\circ} \text { wedge }\right)\end{array}$ & $\begin{array}{c}2 \mathrm{D} \\
(\mathrm{PN})\end{array}$ & $\sim 400$ & $\begin{array}{c}\sim 130 \\
\text { (neutrino) }\end{array}$ & $\sim 600$ \\
\hline $\begin{array}{l}15 M_{\odot}[72] \\
(\mathrm{No})\end{array}$ & $\begin{array}{c}\text { Oak Ridge+ } \\
{[80](2009)}\end{array}$ & $\begin{array}{c}\nu \text {-driven } \\
\left(180^{\circ} \text { wedge }\right)\end{array}$ & $\begin{array}{c}2 \mathrm{D} \\
(\mathrm{PN})\end{array}$ & $\sim 550$ & $\begin{array}{c}\gtrsim 200 \\
\text { (neutrino) }\end{array}$ & $\sim 900$ \\
\hline $\begin{array}{l}25 M_{\odot}[72] \\
(\mathrm{No})\end{array}$ & $\begin{array}{c}\text { Oak Ridge+ } \\
{[80](2009)}\end{array}$ & $\begin{array}{c}v \text {-driven } \\
\left(180^{\circ} \text { wedge }\right)\end{array}$ & $\begin{array}{c}2 \mathrm{D} \\
(\mathrm{PN})\end{array}$ & $\sim 550$ & $\begin{array}{c}\sim 200 \\
\text { (matter) }\end{array}$ & $\sim 900$ \\
\hline
\end{tabular}

Table 2: Similar to Table 1, but for selected GW predictions obtained in the state-of-the-art 2D neutrino-radiation hydrodynamic simulations. See Table 1 for the details of the employed numerical techniques. The column of (Domain) indicates that the computational volume in the lateral direction is solved fully $0 \leq \theta \leq 180^{\circ}$ (" $180^{\circ}$ wedge") or with an assumption of certain imposed symmetry (" $90^{\circ}$ wedge"). $t_{\text {fin }}$ represents the final simulation time, and $\left|h_{\max }\right|$ indicates the absolute maximum GW amplitudes which come either from "neutrino" or "matter" GWs. $f_{\text {peak }}$ represents the peak frequency in the GW spectra. 
emission could be a possible explanation of the increasing trend. It is also worth mentioning that this feature can be seen in the waveform for a rapidly-rotating accretion-induced collapse model [141] of the "Princeton+" simulation (e.g., Figure 10 in [22]).

The top right panel of Figure 3 shows the growth of large scale, non-radial pulsations in the post-shock region (bright regions in the entropy plot $(600 \times 600 \mathrm{~km}))$, which leads to a slower temporal evolution ( $\gtrsim 10 \mathrm{~ms}$ ) of the neutrino GWs (see the line labeled by " $v$ 's" in the top left panel). As seen, an equatorial symmetry was imposed for the $2 \mathrm{D}$ simulation 20 , which is indicated by " $90^{\circ}$ wedge" in Table 2 . Comparing the GW spectra from the sum of matter motions and neutrinos (bottom left panel in Figure 3) to the one only from neutrinos (bottom right), one can clearly see that the low-frequency part of the spectrum (below $\sim 100 \mathrm{~Hz}$ ) is dominated by the contribution of the neutrino GWs. As mentioned above, this is because their wave amplitude varies on much longer timescales $(\gtrsim 10 \mathrm{~ms})$ than that of the mass flow ( $\sim \mathrm{ms}$, e.g., the waveform in the top left panel). For this model, the maximum amplitude comes from the neutrino contribution (e.g., the top left panel and $\left|h_{\max }\right|$ with the comment of (neutrino) in Table 2).

It should be noted whether the maximum amplitude comes from matter or neutrino contribution change from models to models as shown in Table 2 (see the sixth column indicated by (from)), which may reflect the stochastic nature of the SASI and convection which is determined by the non-linear hydrodynamics. In contrast, the peak frequencies in the GW spectrum (typically in the range of 600-1000 Hz) always come from the matter GWs (albeit not explicitly mentioned in the table) due to its short temporal variations ( $f_{\text {peak }}$ in Table 2 ). The bottom panels of Figure 3 show that while the signal-to-noise ratio is probably too small for this event to be detectable by LIGO I, it could be well detected by the Advanced LIGO for a Galactic source. This is a generic feature of the detectability of the GWs from neutrino-driven explosion models.

Figure 4 is the same as Figure 3 but for a non-rotating exploding model of an $11.2 \mathrm{M}_{\odot}$ progenitor of Buras et al. [77]. In contrast to the rotating model mentioned above, no signal is emitted near at bounce for this non-rotating model (compare the insert in the top left of Figure 3 and the waveform near $t=0$ in the top left panel of Figure 4). For this light progenitor model, the wave amplitude from prompt convection (till $\sim 50 \mathrm{~ms}$ after bounce) is very small. Only after the neutrino-driven convection becomes stronger ( $~ 80 \mathrm{~ms}$ after bounce), the maximum (absolute) amplitudes rise to the values of several $10 \mathrm{~cm}$. Although the slower temporal evolution of the neutrino GWs is similar to the one in Figure 3, the monotonically growing trend is not seen for this model. The GW spectrum (bottom panels in Figure 4) is qualitatively quite similar to that of the rotating model (Figure 3), but the GW amplitudes become much smaller. This is probably because of a smaller core mass of the $11.2 M_{\odot}$ progenitor, which could potentially make the mass quadrupole and the emergent neutrino luminosity smaller (compare the spectrum of neutrino GW in the bottom right panel of Figure 3 and 4 .

The left and middle panels in Figure 5 show the wave amplitude (blue line (total), red line (only from neutrinos)) for a non-rotating exploding $15 M_{\odot}$ progenitor in Marek \& Janka (2006) [82] in which a soft variant of the Lattimer-Swesty EOS (left) and a rather stiff Hillebrant-Wolff EOS (middle) was employed 21 Although an overall trend is similar with each other, they found several important EOS effects on the GW signatures. The first one is about the GWs from prompt convection (e.g., the signals before $50 \mathrm{~ms}$ after bounce). As seen, the wave amplitude for the stiffer EOS $(\sim 20 \mathrm{~cm})$ becomes up to a factor of 2 larger maximum amplitudes than its softer EOS

\footnotetext{
${ }^{20}$ Hence the dynamics is north-south symmetric.

${ }^{21}$ An incompressibility at nuclear densities $(K)$ is $180 \mathrm{MeV}$ and $281 \mathrm{MeV}$ for the Lattimer-Swesty and Hillebrant-Wolff EOS, respectively.
} 

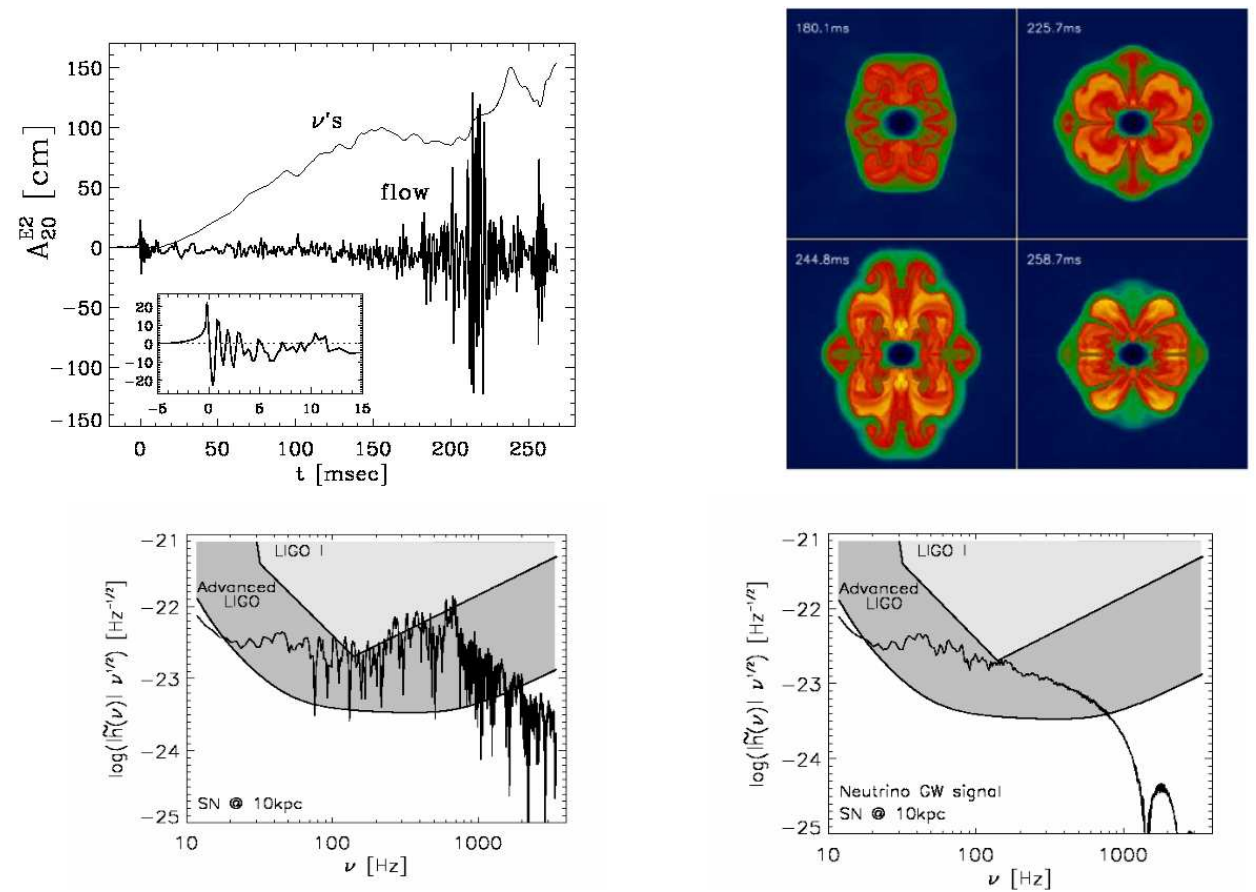

Figure 3: Gravitational waveforms from neutrinos (indicated by " $v$ 's" in the top left panel) or from matter motions (indicated by"flow" in the panel) and their spectra (sum of matter and neutrino (bottom left) and contribution only from neutrino GWs (bottom right)) for a rotating model (s15r) of $15 M_{\odot}$ progenitor in Müller et al. (2004)[120]. The insert in the top left panel shows an enlargement of the signal near bounce, which comes from prompt convection (e.g., section 3.1.1. Top right panel shows the growth of large scale, non-radial pulsations in the post-shock region (bright regions in the entropy plot $(600 \times 600 \mathrm{~km})$ ), which leads to a slower temporal evolution ( $\gtrsim 10 \mathrm{~ms})$ of the neutrino GWs. All of these plots are by courtesy of Müller and the coauthors [120].

counterpart $(\sim 10 \mathrm{~cm})$. They pointed out that the prompt convection exhibits larger amplitude, slightly higher frequencies, and more power for the stiffer EOS. They found that this is because the region of prompt convection involves more mass and extends to larger radii. This may agree with one's intuition that the inner-core mass near bounce becomes larger in the case of stiffer EOS (due to the suppressed electron capture), potentially providing a larger quadrupole GW emission.

The maximum (absolute) amplitudes come from the neutrino contribution irrespective of the employed EOSs (see the column of (from) for the non-rotating $15 M_{\odot}$ progenitor in Table 2). The maximum (absolute) amplitude is about 130 and $60 \mathrm{~cm}$ for the softer and stiffer EOS, respectively 22 The higher neutrino luminosities that are radiated from a more compact PNS in the softer EOS predominantly give a favourable condition for a more efficient emission of the neutrino GWs. However, their peak frequencies are typically below $\sim 100 \mathrm{~Hz}$ (e.g., the bottom right panel in Figure 3), making the component of the neutrino GWs very difficult to be detectable for ground-based detectors whose sensitivity is limited mainly by seismic noises at low

${ }^{22}$ Note that the result only for the softer EOS is given in Table 2. 

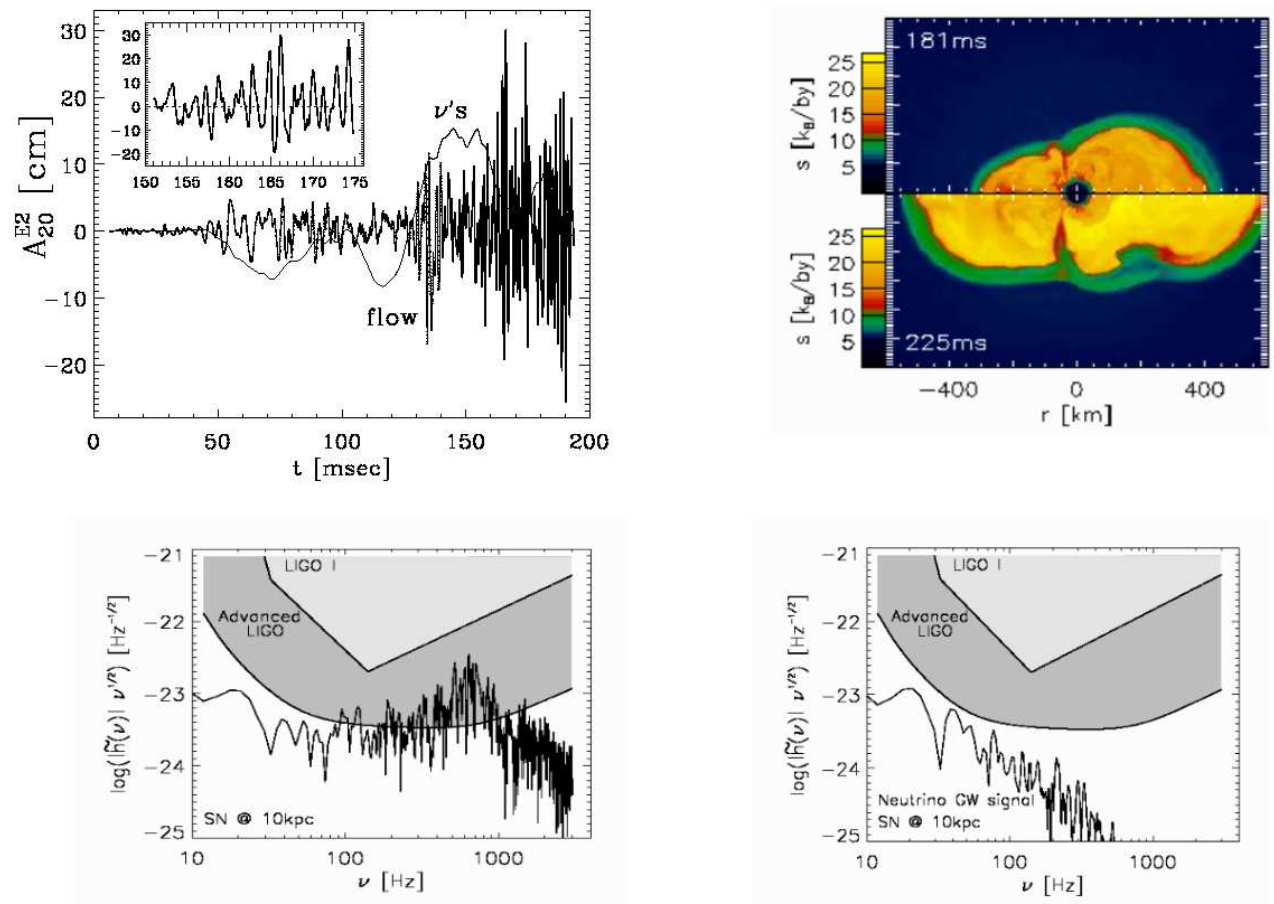

Figure 4: Similar to Figure 3 but for a non-rotating explosion model in the MPA simulations of an $11.2 M_{\odot}$ progenitor (taken from Müller et al. (2004)[120]). The top right panel indicates the success explosion of this model, in which the entropy (bright) with different timescales (181 (top) or $225 \mathrm{~ms}$ (bottom) after bounce) are displayed. The polar axis is directed horizontally. These figures are by courtesy of Müller and the coauthors [120].

frequencies. Therefore the matter GW signals are more important in discussing the detectability. For a Galactic source, the matter signal are of the orders of $10^{-22}$ regardless of the different EOSs. They discussed that they should be marginally observable with LIGO I but it is not easy to tell the EOS difference. This is because although the spectral peak become (slightly) higher if the EOS is soft, this simultaneously sticks out of the highest sensitivity frequency domain of the LIGO instrument.

In contrast to an anticipation in section 3.1.2, their neutrino GWs decrease with time. They argued that this comes from the excess of neutrino emission in the equatorial direction, which was argued to be determined by the anisotropic transport of heavy-lepton neutrinos in the vicinity of the PNS 23. To pin down the GW signal from neutrinos, one apparently needs to precisely determine the neutrino anisotropy which should require multi-angle neutrino transport calculation. Currently it would be practicable in 2D simulations [86] if enough number of momentum-space angles could be cast (probably) by using the next-generation supercomputers. But before that, we may need to be a little bit careful in interpreting the neutrino GWs.

\footnotetext{
${ }^{23}$ On the other hand, they did see the stronger emission in the polar regions regarding $v_{e}$ and $\bar{v}_{e}$, which is consistent with the discussion in section 3.1 .2
} 

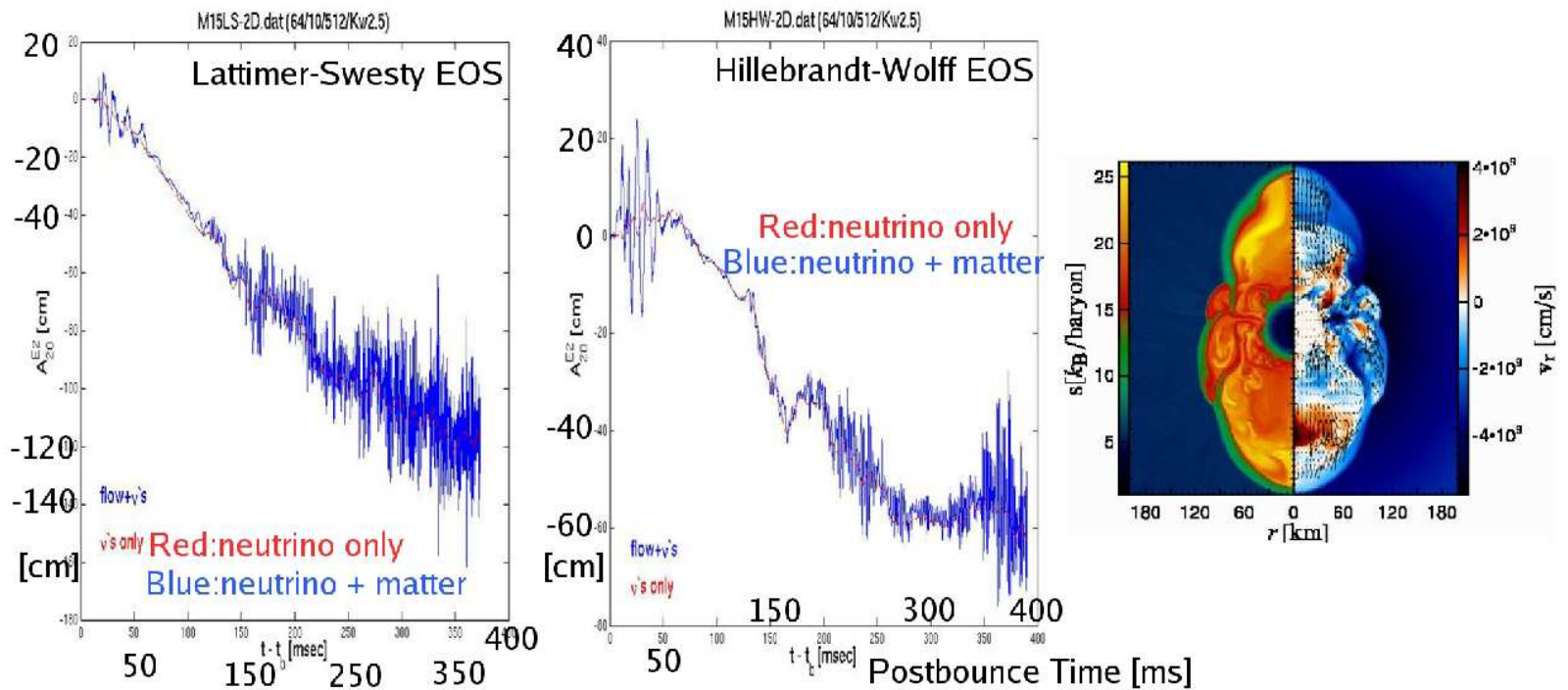

Figure 5: Gravitational waveforms for a non-rotating exploding model in the MPA simulations of a $15 M_{\odot}$ progenitor [82] in which the Lattimer-Swesty (left panel) or Hillebrant-Wolff EOS (middle panel) was employed (taken from Marek et al. (2009) [128]). The right panel indicates the explosion of this model, in which the lefthand panel shows color-coded entropy distribution, the righthand panel the radial velocity components with white and whitish hues denoting matter at or near rest, while black arrows indicate the direction of the velocity fields. These figures are by courtesy of Marek and the coauthors [128].

Keeping this in mind, the neutrino GWs (blue line in the left panel of Figure 6) for a nonrotating $15 M_{\odot}$ exploding model in the OakRidge+ simulation (e.g., Table 1 for the numerical details) show again a monotonically increasing trend. They obtained a similar trend in the waveforms of their exploding models of 12 and $25 M_{\odot}$ progenitors [89]. In consistent with the analysis in section 3.1.1 the matter GWs show a monotonic increase after the SASI-driven sloshing shock turns into 2D prolate explosions ( $\gtrsim 300 \mathrm{~ms}$ postbounce in the left panel of Figure 6). For the first time this model succeeded in capturing the feature, which was not seen in other radiationhydrodynamic simulations. To produce the increasing trend, the anisotropic kinetic energy of the expanding shock needs to be enough large. Relatively earlier shock revival with larger explosion energies (e.g., Table 1) obtained in the OakRidge+ simulations could satisfy the condition. The maximum amplitudes for a Galactic supernova reach to the orders of $10^{-21}$ regardless of the employed progenitors, which could be a promising target for the advanced LIGO (bottom right panel in Figure 6.

Figure 7 shows the wave amplitudes (left panels) with their spectra (middle panels) for a non-rotating (top panels) and rapidly rotating model (bottom panels) for a $13 M_{\odot}$ progenitor 71$]$ obtained in the NAOJ+ simulation (e.g., Table 1 for the numerical details). For the non-rotating model, the neutrino GWs show the increasing trend (green line in the top left panel). This model explodes in a unipolar manner (top right panel), expelling material only in the northern part of the core. Due to this one-sided explosion with smaller mass ejection, this model explodes only weakly with decreasing explosion energies less than $\sim 4 \times 10^{49} \mathrm{erg}$ at the final simulation time (see Figure 4 in [81]). This should be the reason why the matter GW signals (red line in the top 


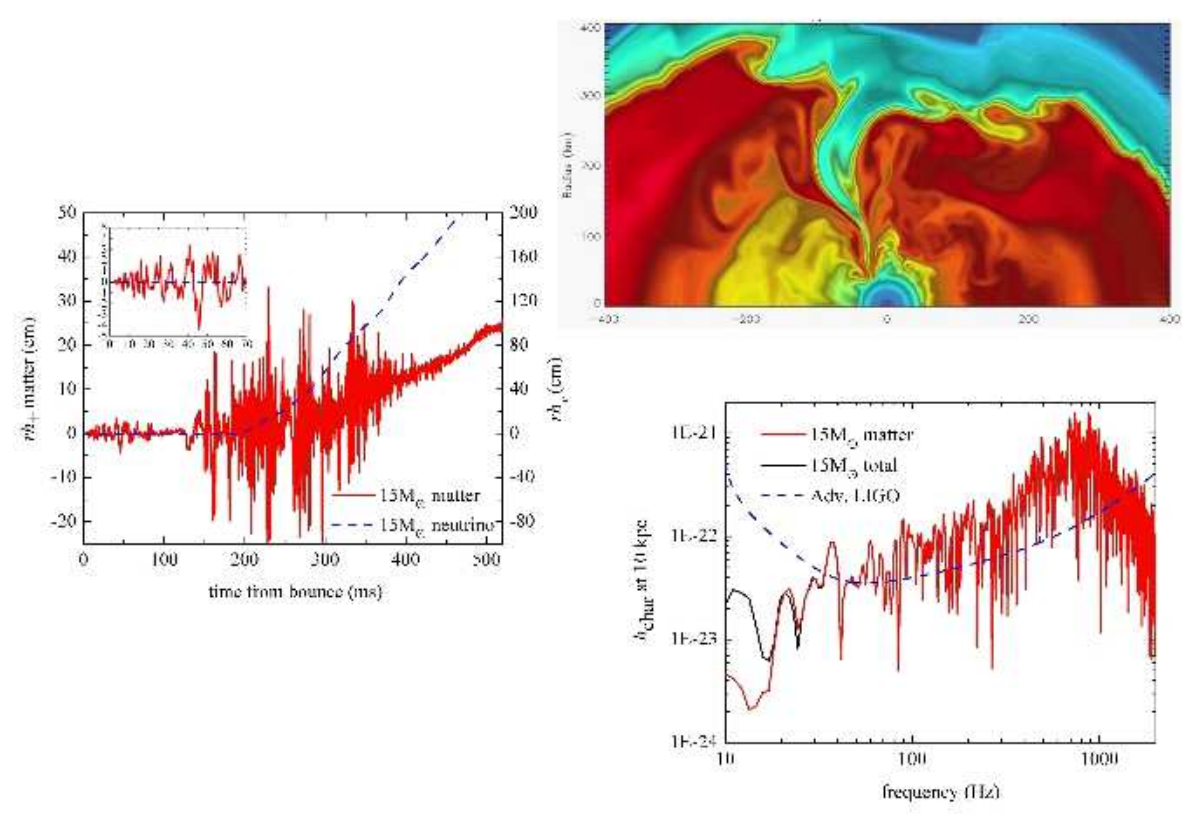

Figure 6: Similar to Figure 3 but for a non-rotating exploding model in the OakRidge+ simulations of a non-rotating 15 $M_{\odot}$ progenitor (from Yakunin et al. (2009) [89]). The left panel shows the gravitational waveforms contributed from matter motions (indicated by matter) and from neutrinos (indicated by neutrino). The insert shows an enlargement of the signal near bounce, which comes from prompt-convection. The top right panel shows entropy distribution at $244 \mathrm{~ms}$ after bounce for the progenitor. A bipolar explosion with high-entropy outflows (yellow-orange-red) is shown along the symmetry axis. The bottom right panel shows the spectra with the sensitivity curve of the advanced LIGO. These figures are by courtesy of Yakunin and the coauthors [89].

left panel) are much smaller than those obtained in the Oak Ridge+ simulation (e.g., red line in Figure 6.

On the other hand, a bipolar explosion is seen to be obtained for their 2D model with rapid rotation 24 (bottom right panel in Figure 7). Since the north-south symmetric $(\ell=2)$ explosion can expel more material than for the unipolar explosion, the explosion energies becomes larger (e.g., Figure 4 in [81]), simultaneously making the matter GWs larger. In fact, the increasing trend in the matter GWs is seen after $~ 400 \mathrm{~ms}$ after bounce (red line in the bottom left panel in Figure 7). The neutrino GWs firstly decrease after bounce (blue line in the same panel), but later shift to exhibit the increasing trend ( $~ 2500 \mathrm{~ms}$ after bounce). The middle panels (for a supernova at a distance of $10 \mathrm{kpc}$ ) show that the spectrum rises to a broad peak between $\sim 600$ and 900 $\mathrm{Hz}$, which could be visible to the advanced-class detectors for the galactic source (see further discussions in [142]).

\footnotetext{
${ }^{24}$ The precollapse angular velocity was taken to be $2 \mathrm{rad} / \mathrm{s}$.
} 

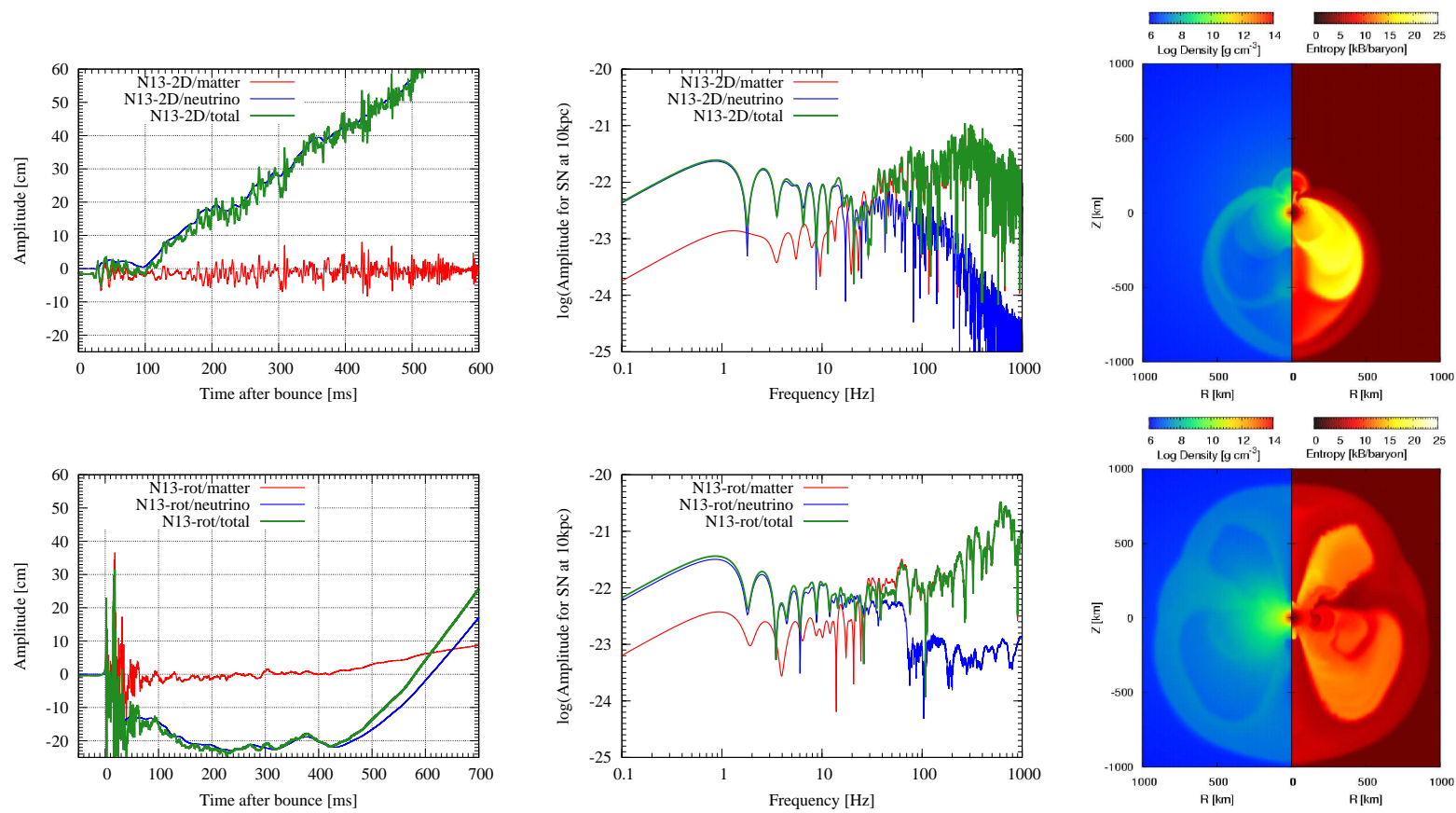

Figure 7: Gravitational waveforms (left panels) with their spectra (middle panels) for a non-rotating (top panels) and rapidly rotating model (bottom panels) for a $13 M_{\odot}$ progenitor [71] obtained in the NAOJ+ simulation (e.g., Table 1). For the rotating model, the precollapse angular velocity is taken to be $2 \mathrm{rad} / \mathrm{s}$. Right panels show snapshots of the density (left half) and the entropy (right half) for models without (top) or with rotation (bottom) at the epoch when the shock reaches $1000 \mathrm{~km}$, corresponding to $\sim 470 \mathrm{~ms}$ after bounce in both cases. The middle panels are for a supernova at a distance of $10 \mathrm{kpc}$. These figures are by courtesy of Suwa and the coauthors [142].

\subsubsection{Stochastic $G W$ nature in experiment $3 D$ simulations}

(By definition) 2D simulations so far mentioned in previous sections have an assumption of axisymmetry. Due to the symmetry axis, the growth of the SASI and the large-scale postshock convection could develop along the axis preferentially, thus suppressing anisotropies in explosions. Since the GW emission is very sensitive to the degree of the explosion anisotropies, 3D simulations are apparently needed for a more accurate GW prediction.

Since it is still computationally very expensive to solve the spectral neutrino transport, a light-bulb scheme (e.g., [54]) has been often employed in 3D simulations to trigger explosions, in which the heating and cooling by neutrinos are treated by a parametric manner (e.g., section 2 and references in [66, 46, 143]). Using the recipe, a handful of GW predictions based on the 3D simulations have been reported so far, which we are going to review in this section. As a preface, we just like to mention that although these studies use experimental approaches and thus their findings should be tested by the first-principle 3D simulations in the future (e.g., [79]), they have indeed shed a new light towards a better understanding of the GW signatures as they have done so in 2D simulations (see discussions in sections 3.1.1 and 3.1.2).

Müller and Janka (1997) [117 coined the first study to analyze the GW signature of 3D non-radial matter motion and anisotropic neutrino emission from prompt convection in the outer 
layers of a PNS during the first $30 \mathrm{~ms}$ after bounce. Their first 3D calculations using the lightbulb recipe were forced to be performed in a wedge of opening angle of $60^{\circ}$. Albeit with this limitation (probably coming from the computer power at that time), they obtained important findings that because of smaller convective activities inside the cone with slower overturn velocities, the GW amplitudes of their 3D models are more than a factor of 10 smaller than those of the corresponding 2D models, and the wave amplitudes from neutrinos are a factor of 10 larger than those due to non-radial matter motions. With another pioneering (2D) study by Burrows \& Hayes (1996) [118], it is worth mentioning that those early studies had brought new blood into the conventional GW predictions, which illuminated the importance of the theoretical prediction of the neutrino GWs.

A series of findings obtained by Fryer et al. in early 2000s [55, 144] have illuminated also the importance of the 3D modeling. By running their 3D Newtonian Smoothed Particle Hydrodynamic (SPH) code coupled to a gray flux-limited neutrino transport scheme, they studied the GW emission due to the inhomogeneous core-collapse, core rotation, low-modes convection, and anisotropic neutrino emission. Although the early shock-revival and the subsequent powerful explosions obtained in these SPH simulations have yet to be confirmed by other groups, their approach paying particular attention to the multiple interplay between the explosion dynamics, the GW signatures, the kick and spins of pulsars, and also the non-spherical explosive nucleosynthesis, blazed a new path on which current supernova studies are progressing.

We also studied the GW signals from 3D models that mimic neutrino-driven explosions aided by the SASI [126, 127, 145]. These studies were an extension of the 2D simulations already mentioned in section 3.1.2 in which the light-bulb scheme was used to obtain explosions and the initial conditions were derived from a steady-state approximation of the postshock structure and the dynamics only outside an inner boundary at $50 \mathrm{~km}$ was solved. The left panel of Figure 8 shows the evolution of 3D hydrodynamic features from the onset of the non-linear regime of SASI (top left) until the shock break-out (bottom right) with the gravitational waveform from neutrinos inserted in each panel. As seen, the major axis of the growth of SASI is shown to be not aligned with the symmetric axis ( $: Z$ axis in the figure) and the flow inside the standing shock wave is not symmetric with respect to this major axis (see the first and third quadrant). This is a generic feature obtained in their 3D models, which is in contrast to the axisymmetric case. As discussed in section 3.1.2 the GW amplitudes from the SASI in 2D showed an increasing trend with time due to the symmetry axis, along which SASI can develop preferentially. Free from such a restriction, a variety of the waveforms is shown to appear (see waveforms inserted in Figure 8). Furthermore, the 3D standing shock can also oscillate in all directions, which leads to the smaller explosion anisotropy than 2D. With these two factors, the maximum amplitudes seen either from the equator or the pole becomes smaller than 2D. On the other hand, their sum in terms of the total radiated energy are found to be almost comparable between 2D and 3D models, which is likely to imply the energy equipartition with respect to the spatial dimensions.

The right panel of Figure 8 shows the gravitational waveforms for models with different neutrino luminosity. The input luminosity for the bottom panel is smaller than that for the top panel only by $0.5 \%$. Despite the slight difference, the waveforms of each polarization are shown to exhibit no systematic similarity when seen either from the pole or equator.

The stochastic nature of GWs produced by the interplay of the SASI and neutrino-driven explosions have been also confirmed more recently by Müller et al. (2011) [131]. They analyzed the GW signatures based on their 3D models of parametric neutrino-driven explosions [146] 

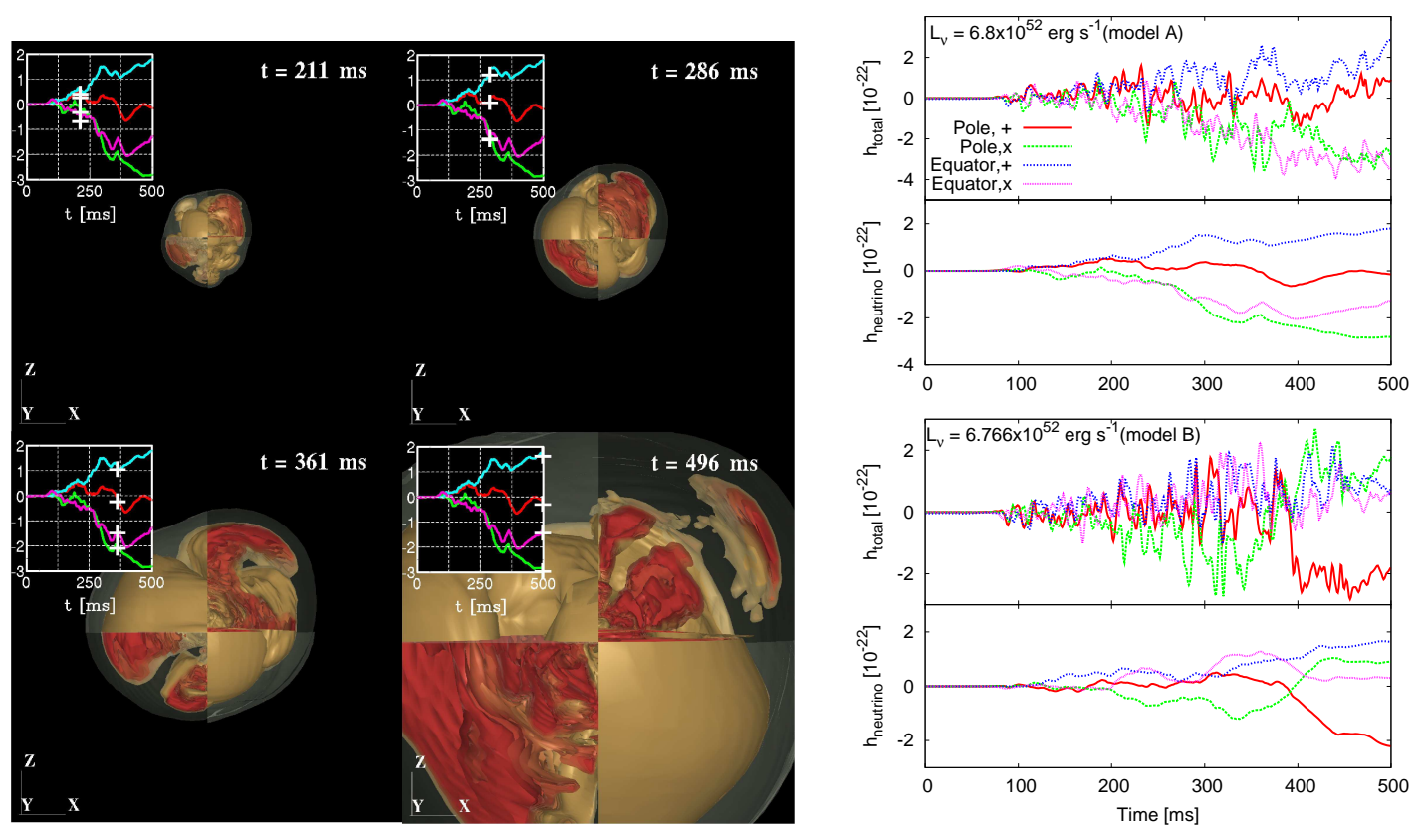

Figure 8: Left panel shows four snapshots of the entropy distributions of a representative 3D supernova explosion model (corresponding to model A in Kotake et al. (2009) [127]). The second and fourth quadrant of each panel shows the surface of the standing shock wave. In the first and third quadrant, the profiles of the high entropy bubbles (colored by red) inside the section cut by the $Z X$ plane are shown. The side length of each plot is $1000 \mathrm{~km}$. The insets show the gravitational waveforms from anisotropic neutrino emissions, with ' + ' on each curves representing the time of the snapshot. Note that the colors of the curves are taken to be the same as the top panel. After about $100 \mathrm{~ms}$, the deformation of the standing shock becomes remarkable marking the epoch when the SASI enters the non-linear regime. At the same time, the gravitational amplitudes begin to deviate from zero. Right panel shows gravitational waveforms from neutrinos (bottom) and from the sum of neutrinos and matter motions (top), seen from the polar axis and along the equator (indicated by 'Pole' and 'Equator') with polarization (+ or $\times$ modes) for two representative 3D models of A and B (see [127] for details), in which the input luminosity for the two pair panels differs only $0.5 \%$. From the right panels, it can be seen that the overall structures of the waveforms are predominantly determined by the neutrino GWs which was also the case in 2D simulations (e.g., section 3.1.2. The distance to the source is assumed to be $10 \mathrm{kpc}$. 
in which the gray spectral neutrino transport is solved in a ray-by-ray manner [5925. At the sacrifice of cutting out the high-density core (at around $80-120 \mathrm{~km}$ in radius), their 3D models succeeded in following the dynamics from the post-bounce accretion phase through the onset of the explosion more than one second for the first time. Among a number of interesting findings obtained in their work, we here focus on the progenitor dependence that could not be studied by Kotake et al. $(2009,2011)$ [127, 145] who used the idealized initial conditions.

The top two panels in Figure 9 show snapshots of blast morphologies between two models, in which a non-rotating $15 M_{\odot}$ progenitors either evolved by Woosley and Weaver (1995) (left) or by Limongi et al. (1995) (right) was employed, respectively. Note that they succeeded in following an unprecedentedly long-term 3D evolution (up to $1.4 \mathrm{~s}$ after bounce) by utilizing an axis-free coordinate system; the so-called Yin-Yang grids (see [146] for more details). The middle panels in Figure 9 indicate that the total GW amplitude is significantly different from that of the flow-only GW amplitudes (not shown) for models which do or do not exhibit PNS convection below the neutrino sphere. Particularly at late times ( $t \gtrsim 0.8 \mathrm{~s}$ in the middle panels), anisotropic neutrino emission causes a continuing growth of the GW amplitudes for the model using the Woosley-Weaver (indicated by W15) progenitor (left) instead of saturation seen for the model using the Limongi progenitor ( indicated by L15, right panel). By a detailed spectrogram analysis of the GW energy distributions (see their Figure 12), they revealed that the primary agent to make the model discrepancy is the PNS convection, which develops much more vigorously for the W15 progenitor model than for the L15 progenitor model. This can be also depicted in the bottom panels of Figure 9 which shows bigger neutrino anisotropy for the W15 progenitor model (left) in the late postbounce phase $(t \gtrsim 0.8 \mathrm{~s})$ than for the L15 progenitor model (right). They furthermore pointed out that in their 3D models, very prominent, quasi-periodic sloshing motions due to the SASI are absent and the emission from different surface areas facing an observer adds up incoherently, so that the measurable modulation amplitudes of the GW signals (as well as neutrinos) are significantly smaller than predicted by 2D simulations ([129, 89]).

The effects of stellar rotation on the stochastic nature of the GWs have been recently studied by Kotake et al. (2011) [145] who used the same numerical techniques that was already mentioned in explaining Figure 8, The top two panels in Figure 10 show the gravitational waveform for a typical 3D with rotation (left:total amplitudes, right:neutrino only). To construct a model with rotation, a uniform rotation was manually given on the flow advecting from the outer boundary of the iron core as in 67], whose specific angular momentum is assumed to agree with recent stellar evolution models [84]. Comparing to the right panel in Figure 8] (i.e., in the absence of rotation), one can clearly see a sudden rise in the GW amplitude after around $500 \mathrm{~ms}$ for the rotating model (blue line in the top panels in Figure 10), which is plus mode of the neutrino GWs seen from the equator. These features were found to be common in the fifteen 3D models computed in their study.

Seen from the equatorial direction (the middle left panel of Figure 10), one may guess the presence of the sloshing modes, but it just happens to develop along the rotational axis ( $z$-axis) at this epoch. It should be emphasized that the dominance of $h_{v,+}^{\text {equ }}$ observed in the current 3D simulations have nothing to do with the one discussed in section 3.1.2. Free from the 2D axis effects, the major axis of the SASI changes stochastically with time, and the flow patters behind the standing shock also change in every direction. As a result, the sloshing modes can make only a small contribution to the $\mathrm{GW}$ emission. The remaining possibility is that the spiral flows seen

\footnotetext{
${ }^{25}$ It is worth mentioning that a complete derivation of formulae for extracting the neutrino GWs is given for arbitrary positioning between the source and observer coordinate systems (e.g., section 4 in [131]).
} 

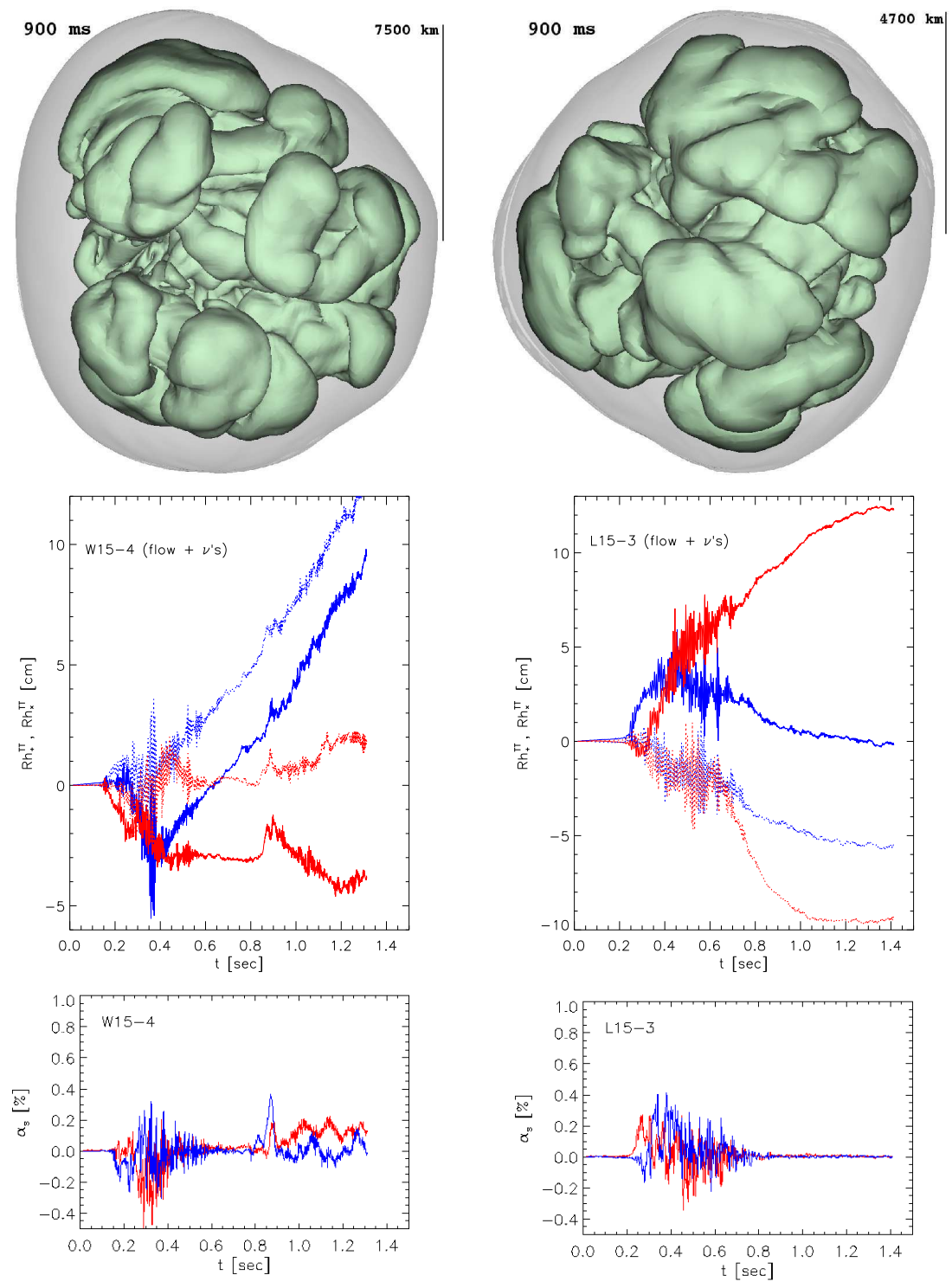

Figure 9: Top two panels show snapshots illustrating a globally asymmetric 3D explosion in the post-accretion phase of two different progenitor models using the Woosley-Weaver (left) or Limongi progenitor (right) of a $15 M_{\odot}$ progenitor (taken from Müller et al. (2011) [131]). Note that "-4" or "-3" differ only by the initial seed perturbations (see [131] for more details). The time and linear scale are indicated in each plot. Middle panels show the plus (blue) or cross (red) mode of the wave amplitude due to anisotropic mass flow and neutrino emission for models W15-4 (left) and L15-3 (right), respectively. The solid curves show the amplitudes for an observer above the north pole of the source, while the other curves give the amplitudes at the equator. Similar to the middle panels, the bottom panels shows the anisotropy parameter of neutrino emission (only the plus mode is shown). These figures are by courtesy of Müller and the coauthors [131]. 

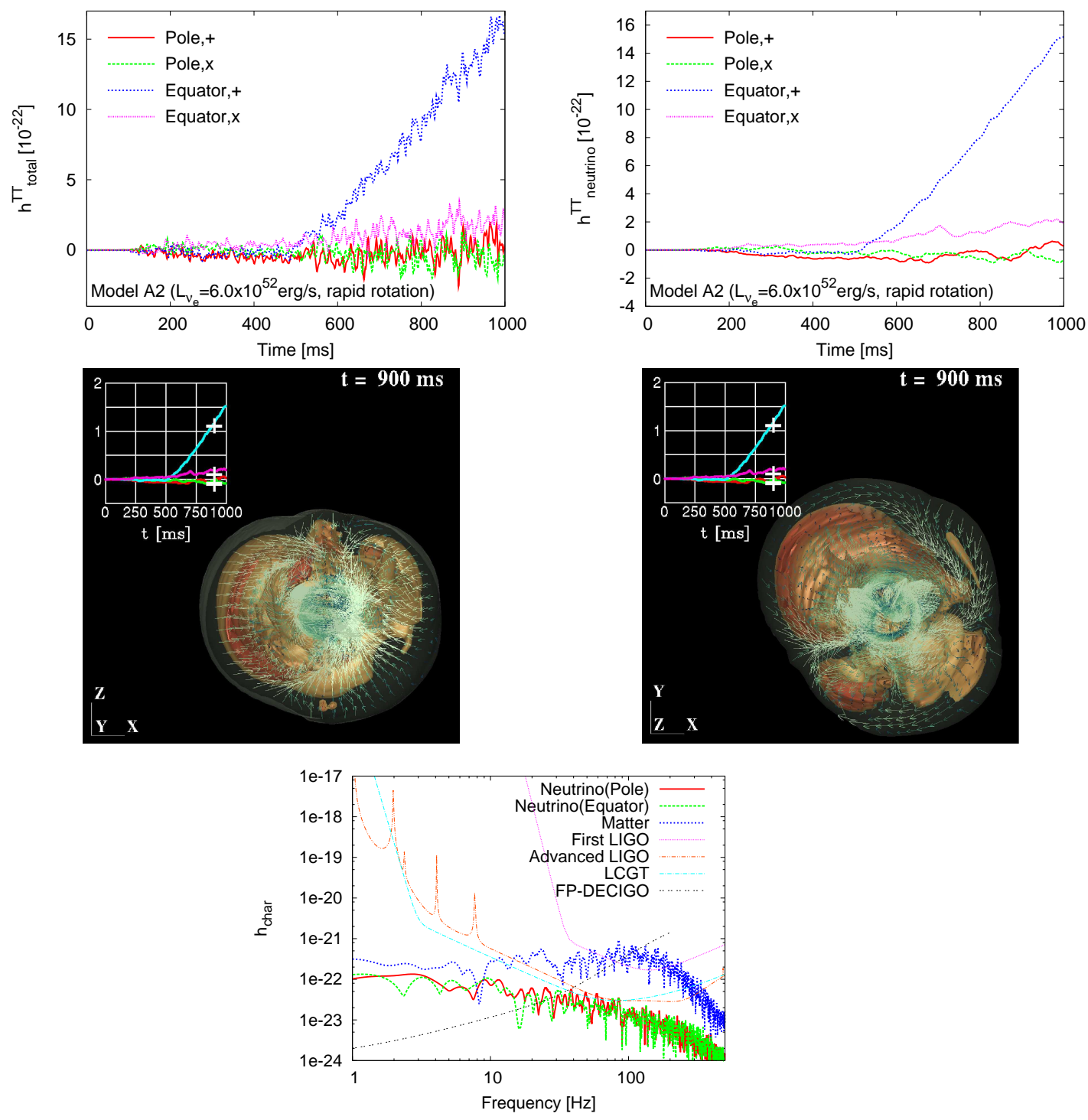

Figure 10: Top panels show gravitational waveforms from the sum of neutrinos and matter motions (left) and only from neutrinos (right) obtained in a parametric explosion 3D model with rotation (taken from Kotake et al. (2011) [145]). Similar to the left panel in Figure 8 the middle panels show a partial cutaway of the entropy isosurfaces and the velocity vectors on the cutting plane for the equatorial (left) and polar observer (right), respectively. The bottom panel shows spectral distributions for the model from matter motions ("Matter") and neutrino emission ("Neutrino") seen from the pole or the equator (e.g., [145]) with the expected detection limits of TAMA300 [11], first LIGO and advanced LIGO [147], LCGT [15], and Fabry-Perot type DECIGO [148]. The distance to the supernova is assumed to be $10 \mathrm{kpc}$. Note that for the matter signal, the + mode seen from the polar direction is plotted. 
in the middle right panel should be a key importance to understand the GW feature mentioned above. In fact, by analyzing the matter distribution on the equatorial plane, it was found that the compression of matter is more enhanced in the vicinity of the equatorial plane due to the growth of the spiral SASI modes, leading to the formation of the spiral flows circulating around the spin axis with higher temperatures. As a result, the neutrino emission seen parallel to the spin axis becomes higher than the ones seen from the other direction. Remembering that the lateral-angle $(\theta)$ dependent function of the GW formulae (e.g., in Equation (10) is positive near the north and south polar caps, the dominance of the polar neutrino luminosities leads to make the positively growing feature of $h_{v,+}^{\text {equ }}$ in the top panels of Figure 10 (blue line). From the spectral analysis of the gravitational waveform (the bottom panel of Figure 10), it can be readily seen that it is not easy to detect these neutrino-originated GW signatures with slower temporal evolution ( $\gtrsim O(10) \mathrm{ms})$ by ground-based detectors whose sensitivity is limited mainly by the seismic noises at such lower frequencies. However these signals may be detectable by the recently proposed future space interferometers like Fabry-Perot type DECIGO ([148], black line in the bottom panel). Contributed by the neutrino GWs in the lower frequency domains, the total GW spectrum tends to become rather flat over a broad frequency range below $\sim 100 \mathrm{~Hz}$. These GW features obtained in the context of the SASI-aided neutrino-driven mechanism are different from the ones expected in the other candidate supernova mechanism, such as the MHD mechanism (e.g., section 3.2) and the acoustic mechanism ([123], e.g., section 7.1 in [22]). Therefore the detection of such signals could be expected to provide an important probe into the explosion mechanism.

Finally, most of the 3D models summarized in this section cut out the PNS and the neutrino transport is approximated by a simple light-bulb scheme [145] or by the gray transport scheme [131]. Needless to say, these exploratory approaches are but the very first step to model the neutrino-heating explosion and to study the resulting GWs. As already mentioned, the excision of the central regions inside PNSs truncates the feedback between the mass accretion to the PNS and the resulting neutrino luminosity, which should affect the features of the neutrino GWs. By the cut-out, efficient GW emission of the oscillating neutron star [123] and non-axisymmetric instabilities [122, 111, 112] of the PNSs, and the enhanced neutrino emissions inside the PNSs [128] cannot be treated in principle. To elucidate the GW signatures in a much more quantitative manner, full 3D simulations with a spectral neutrino transport are apparently needed. This is unquestionably a vast virgin territory awaited to be explored for the future.

\subsection{GWs from MHD Explosions}

As already mentioned in the beginning of section 3 , state-of-the-art results concerning the GW signatures emitted near bounce in the case of rapidly rotating core-collapse have been given in the recent review by Ott (2009) [22] (e.g., section 4). Conventionally the waveforms of the bounce signals are categorized into the three types, namely types I, II, and III. As explained in Ott (2009) [22], type II and III waveforms are shown less likely to appear than type I, because a combination of general relativity and electron capture near core bounce suppresses multiple bounce in the type II waveforms [109, 106, 107]. In general, a realistic nuclear equation of state (EOS) is stiff enough to forbid the type III waveforms. So the generic type of the bounce signals is now known to take the type I waveform.

For avoiding overlap, we mainly focus on the postbounce GW emission in the context of rapidly rotational core-collapse in this section. The major GW emission cites have been proposed to be magnetohydrodynamic (MHD) outflows and nonaxisymmetric instabilities, which will be separately described in sections 3.2.1 and 3.2.2, respectively. 


\subsubsection{MHD outflows}

Numerical simulations of MHD stellar explosions have a long history26 started already in early 1970's by LeBlanc and Wilson (1970) [149] shortly after the discovery of pulsars [150, 151, 152]. However, it is rather only recently that the MHD studies come back to the frontend topics in the supernova research followed by a number of extensive MHD simulations (e.g., [153, 154, 103, 155, 156, 157, 158, 159, 160, 161, 162, 137, 163, 164, 165, 166] for references therein). Main reasons for this activity are observations indicating very asymmetric explosions [25, 26], and the interpretation of magnetars [167, 168] and gamma-ray bursts (e.g., [114, 115]) as a possible outcome of the magnetorotational core-collapse of massive stars.

The MHD mechanism of stellar explosions relies on the extraction of rotational free energy of collapsing progenitor core via magnetic fields. Hence a high angular momentum of the core is preconditioned for facilitating the mechanism [169]. Given (a rapid) rotation of the precollapse core, there are at least two ways to amplify the initial magnetic fields to a dynamically important strength, namely by the field wrapping by means of differential rotation that naturally develops in the collapsing core, and by the magnetorotational instability (MRI, see [170]).

In the case of canonical initial magnetic fields $\left(\sim 10^{9} \mathrm{G}\right)[84]$, the fastest growing modes of the MRI are estimated to be at most several meters in the collapsing iron core [171]. At present, it is generally computationally too expensive to resolve those small scales in the global MHD simulations, typically more than two or three orders-of-magnitudes smaller than their typical finest grid size. Let us remind first that the MHD explosions presented in the rest of this section are predominantly generated by the field wrapping mechanism assuming a very strong precollapse magnetic field $\left(B_{0} \gtrsim 10^{11-12} \mathrm{G}\right)$. Recent stellar evolution calculations show that this extreme condition could be really the case, albeit minor ( $\sim 1 \%$ of massive star population [172]), for progenitors of rapidly rotating metal-poor stars, which experience the so-called chemically homogeneous evolution [114, 115].

Figures 11 to 13 show several examples of gravitational waveforms obtained in representative MHD explosion models, in which the left and right panel shows the waveform and the blast morphology, respectively. After the bounce and ring-down signals with their typically duration of $\sim 10 \mathrm{~ms}$ after bounce, a quasi-monotonically growing trend can be seen in the every waveform in the left panels of Figure 11 to 13

Figures 11 and 12 are from Obergaulinger et al. (2006) and Shibata et al. (2006) who obtained 2D MHD explosions either in Newtonian and approximate GR or full GRMHD simulations, respectively. Both of them employed polytropic precollapse models, a phenomenological EOS that mimics deleptonization and neutrino cooling. They pointed out that the increasing trend comes from bipolar flows driven by MHD explosions, which can be visible only for cores with precollapse magnetic fields over $B_{0} \gtrsim 10^{12} \mathrm{G}$. 3D MHD simulations by Scheidegger et al. (2010) [112] included realistic EOSs and a deleptonization effect based on 1D-Boltzmann simulations [113]. Compared to the corresponding 2D models, they pointed out that the jet-like explosions in their 3D models are much more difficult to obtain because the wind-up of the poloidal into the toroidal field does not proceed efficiently enough due to the growth of spiral SASI modes. But when a very rapid precollapse angular velocity of $\Omega_{0}=3 \pi \mathrm{rad} / \mathrm{s}$ and (strong) magnetic field of $B_{0}=10^{12} \mathrm{G}$ were assumed, they observed the secularly growing trend associated with the MHD explosions also in their 3D simulations (Figure 13).

In most of the mentioned MHD simulations, the bounce shock generally does not stall and a prompt explosion occurs within a few ten milliseconds after bounce. In fact, the typical

\footnotetext{
${ }^{26}$ almost as old as the neutrino-driven model in Colagate and White (1966).
} 

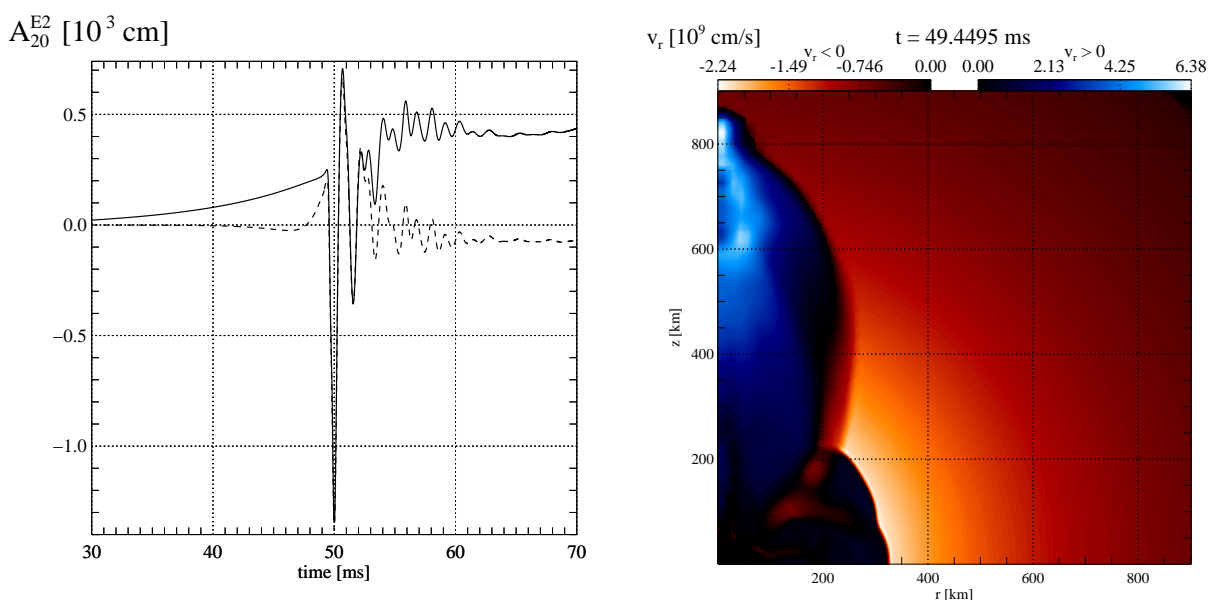

Figure 11: Left panel shows a gravitational waveform in the 2D post-Newtonian simulations by Obergaulinger et al. (2006) [159] for their model A1B3G3-D3M13 in terms of the quadrupole amplitude $A_{20}^{E 2}$. The dashed line comes from the contribution of regions inside $60 \mathrm{~km}$ in radius while the solid line shows the total signal. Comparing to the right panel (velocity contours at $\sim 50$ postbounce), the postbounce offset in the waveform is shown to come from the outer regions with bipolar outflows. These figures are by courtesy of Obergaulinger and the coauthors.
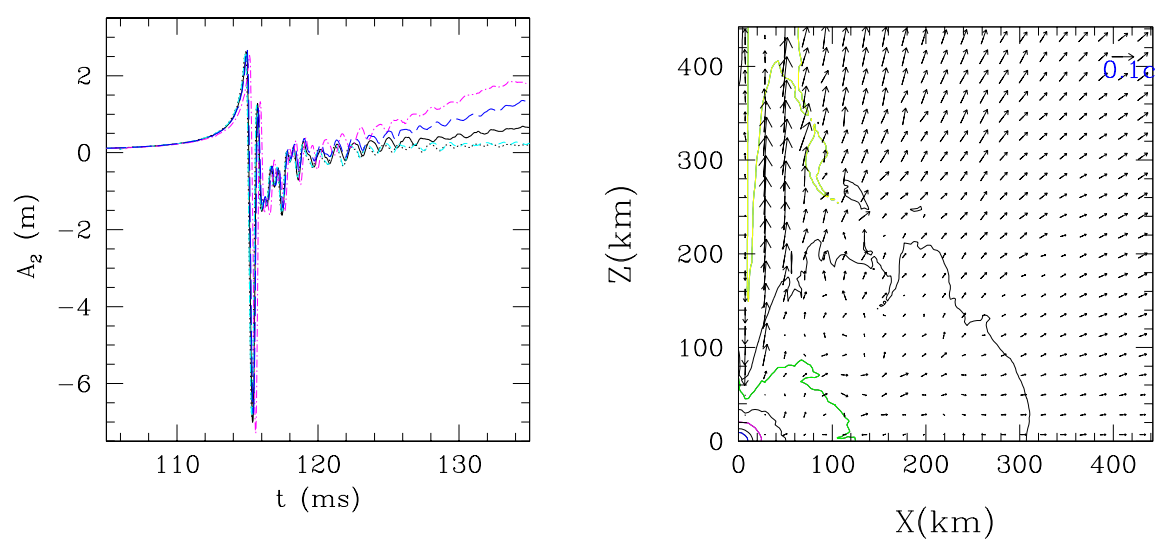

Figure 12: Left panel shows a gravitational waveform in the 2D GRMHD simulations by Shibata et al. (2006) [173] for their models A1 to A4. For models with stronger precollapse magnetic fields (pink and blue lines), the quasi-increasing trend can be clearly seen. Right panel shows density contours with velocity vectors for a snapshot $(t=152 \mathrm{~ms}$ for model A4) illustrating MHD-driven jet-like outflows. These figures are by courtesy of Shibata and the coauthors. 

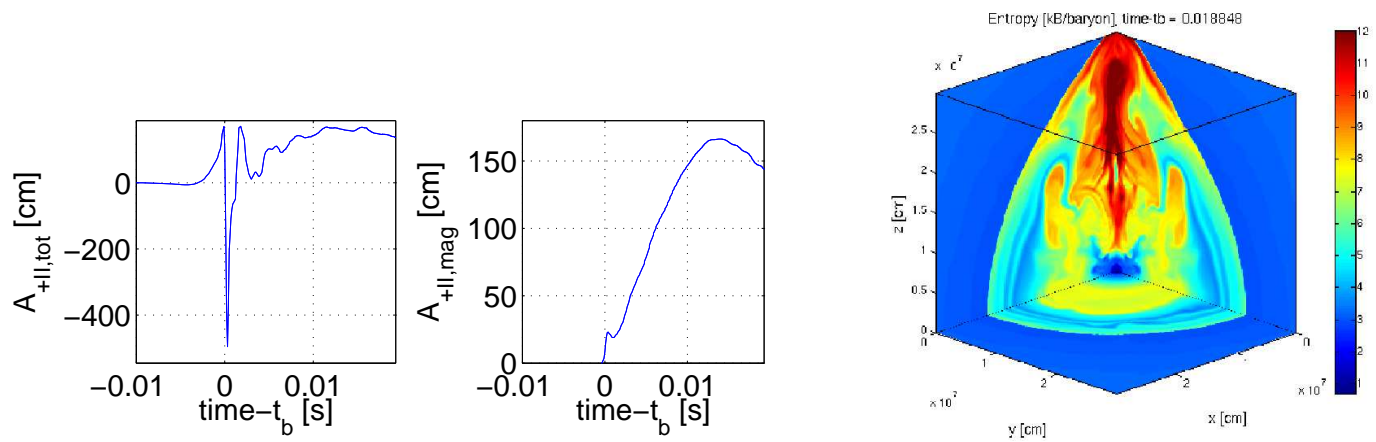

Figure 13: Left pair panels show gravitational waveform in the 3D post-Newtonian simulations by Scheidegger et al. 2010) [112] for their model R4E1CF in which the left- and right- handside corresponds to the total amplitude and contribution only from the magnetic field, respectively. The right panel shows the 3D blast morphology of their MHD explosion (entropy distribution at $\sim 18 \mathrm{~ms}$ postbounce for a cubic of $300^{3} \mathrm{~km}^{3}$ ). These figures are by courtesy of Scheidegger and the coauthors.

timescales shown in Figures 11 to 13 are at most $20 \mathrm{~ms}$ after bounce. This is because some of them followed adiabatic core-collapse [159, 173], in which a polytropic EOS is employed to mimic supernova microphysics, and the deleptonization scheme in [112] cannot capture a drastic energy-loss behind the bounce shock due to neutrino cooling. However, for models with weaker precollapse magnetic fields akin to the current GRB progenitors, the prompt shocks stall firstly in the core like a conventional supernova model with more sophisticated neutrino treatment (e.g., the MHD version of the Princeton+ simulations presented in [160]). In such a case, the onset of MHD explosions, depending on the initial rotation rates, can be delayed till $100 \mathrm{~ms}$ after bounce [160, 164].

Takiwaki and Kotake (2011) [174] addressed this issue by performing 2D special relativistic MHD (SRMHD) simulations with the use of an approximate GR potential [158] in which a neutrino leakage scheme was employed to mimic neutrino cooling. The top left panel of Figure 14 shows the quasi-monotonically increasing trend, which is obtained for a model with strong precollapse magnetic field $\left(B_{0}=10^{12} \mathrm{G}\right)$ also with rapid rotation initially imposed $(\beta$ parameter $=$ $0.1 \%$ with $\beta$ representing ratio of the rotational energy to the absolute value of the gravitational energy prior to core-collapse). Such a feature cannot be observed for a weakly magnetized model $\left(B_{0}=10^{11} \mathrm{G}\right.$. top right panel).

To understand the origin of the increasing trend, it is most straightforward to look into the quadrupole GW formula, which can be expressed in 2D as [175],

$$
h=\frac{1}{R} A_{20}^{E 2} \frac{1}{8} \sqrt{\frac{15}{\pi}} \sin ^{2} \theta,
$$

where $R$ is the distance to the source, $\theta$ is the viewing angle of the source, and $A_{20}^{\mathrm{E} 2}$ is the quadrupole harmonic amplitude consisting of the following three parts $[103,154,158,174]$,

$$
A_{20}^{\mathrm{E} 2}=A_{20 \text { (hyd) }}^{\mathrm{E} 2}+A_{20(\text { mag })}^{\mathrm{E} 2}+A_{20 \text { (grav) }}^{\mathrm{E} 2} .
$$

On the right hand side, the first term is related to anisotropic kinetic energies which we refer to 
as hydrodynamic part,

$$
\begin{aligned}
A_{20}^{\mathrm{E} 2} \text { (hyd) } & =\frac{G}{c^{4}} \frac{32 \pi^{3 / 2}}{\sqrt{15}} \int_{0}^{1} d \mu \int_{0}^{\infty} r^{2} d r f_{20}^{\mathrm{E} 2} \text { (hyd) } \\
f_{20}^{\mathrm{E} 2} \text { (hyd) } & =\rho_{*} W^{2}\left(v_{r}^{2}\left(3 \mu^{2}-1\right)+v_{\theta}^{2}\left(2-3 \mu^{2}\right)-v_{\phi}^{2}-6 v_{r} v_{\theta} \mu \sqrt{1-\mu^{2}}\right),
\end{aligned}
$$

where $\rho_{*}$ is effective density $\rho_{*}=\rho+\left(e+p+|b|^{2}\right) / c^{2}$ with $\rho, e, p$ and $b^{2}=b_{i} b^{i}$ representing the baryon density, internal energy, pressure, and the magnetic energy, respectively. $\mu=\cos \theta$ is the direction cosine and $W=1 / \sqrt{1-v^{k} v_{k}}$ is the Lorentz boost factor with $v_{i}$ representing the spacial velocities in the spherical coordinates (see [174] for more details). The second term is related to anisotropy in gravitational potentials which we call as the gravitational part,

$$
\begin{aligned}
A_{20 \text { (grav) }}^{\mathrm{E} 2}= & \frac{G}{c^{4}} \frac{32 \pi^{3 / 2}}{\sqrt{15}} \int_{0}^{1} d \mu \int_{0}^{\infty} r^{2} d r f_{20}^{\mathrm{E} 2} \text { (grav) } \\
f_{20 \text { (grav) }}^{\mathrm{E} 2}= & {\left[\rho h\left(W^{2}+\left(v_{k} / c\right)^{2}\right)+\frac{2}{c^{2}}\left(p+\frac{|b|^{2}}{2}\right)-\frac{1}{c^{2}}\left(\left(b^{0}\right)^{2}+\left(b_{k}\right)^{2}\right)\right] } \\
& \times\left[-r \partial_{r} \Phi\left(3 \mu^{2}-1\right)+3 \partial_{\theta} \Phi \mu \sqrt{1-\mu^{2}}\right],
\end{aligned}
$$

where $\Phi$ is the gravitational potential of self-gravity, and finally the third term is related to anisotropy in magnetic energies that we refer to as magnetic part,

$$
\begin{aligned}
A_{20(\mathrm{mag})}^{\mathrm{E} 2} & =-\frac{G}{c^{4}} \frac{32 \pi^{3 / 2}}{\sqrt{15}} \int_{0}^{1} d \mu \int_{0}^{\infty} r^{2} d r f_{20}^{\mathrm{E} 2}(\mathrm{mag}) \\
f_{20(\mathrm{mag})}^{\mathrm{E} 2} & =\left[b_{r}{ }^{2}\left(3 \mu^{2}-1\right)+b_{\theta}{ }^{2}\left(2-3 \mu^{2}\right)-b_{\phi}{ }^{2}-6 b_{r} b_{\theta} \mu \sqrt{1-\mu^{2}}\right] .
\end{aligned}
$$

The middle right panel of Figure 14 shows contributions to the total GW amplitudes (equation (6)) for the strongly magnetized model (corresponding to the top left panel in Figure 14], in which the left-hand-side panels are for the sum of the hydrodynamic and gravitational part,

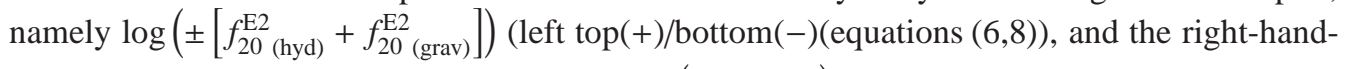
side panels are for the magnetic part, namely $\log \left( \pm f_{20}^{\mathrm{E} 2}(\mathrm{mag})\right)($ right top $(+) /$ bottom(-)) (e.g., equation (10)). By comparing to the middle left panels, it can be seen that the positive contribution is overlapped with the regions where the MHD outflows exist. The major positive contribution is from the kinetic term of the MHD outflows with large radial velocities (e.g., $+\rho_{*} W^{2} v_{r}^{2}$ in equation (6)). The magnetic part also contributes to the positive trend (see top right-half in the middle right panel (labeled by mag $(+))$ ). This comes from the toroidal magnetic fields (e.g., $+b_{\phi}^{2}$ in equation (10), which dominantly contributes to drive MHD explosions.

The bottom panels in Figure 14 show the GW spectra for a pair of models that does or does not have the increasing trend (left and right) as in the top panels. Regardless of the increasing trend, the peak amplitudes in the spectra are rather broad-band around $100-1000 \mathrm{~Hz}$. On the other hand, the spectra for lower frequency domains (below $\sim 100 \mathrm{~Hz}$ ) are much larger for the model with the increasing trend (left) than without (right). This reflects a slower temporal variation of the secular drift inherent to the increase-type waveforms (e.g., top panels in Figure 147. Similar to the neutrino GWs mentioned in section 3.1, it is true that the GWs in the low frequency domains are difficult to detect due to seismic noises, but a recently proposed future 

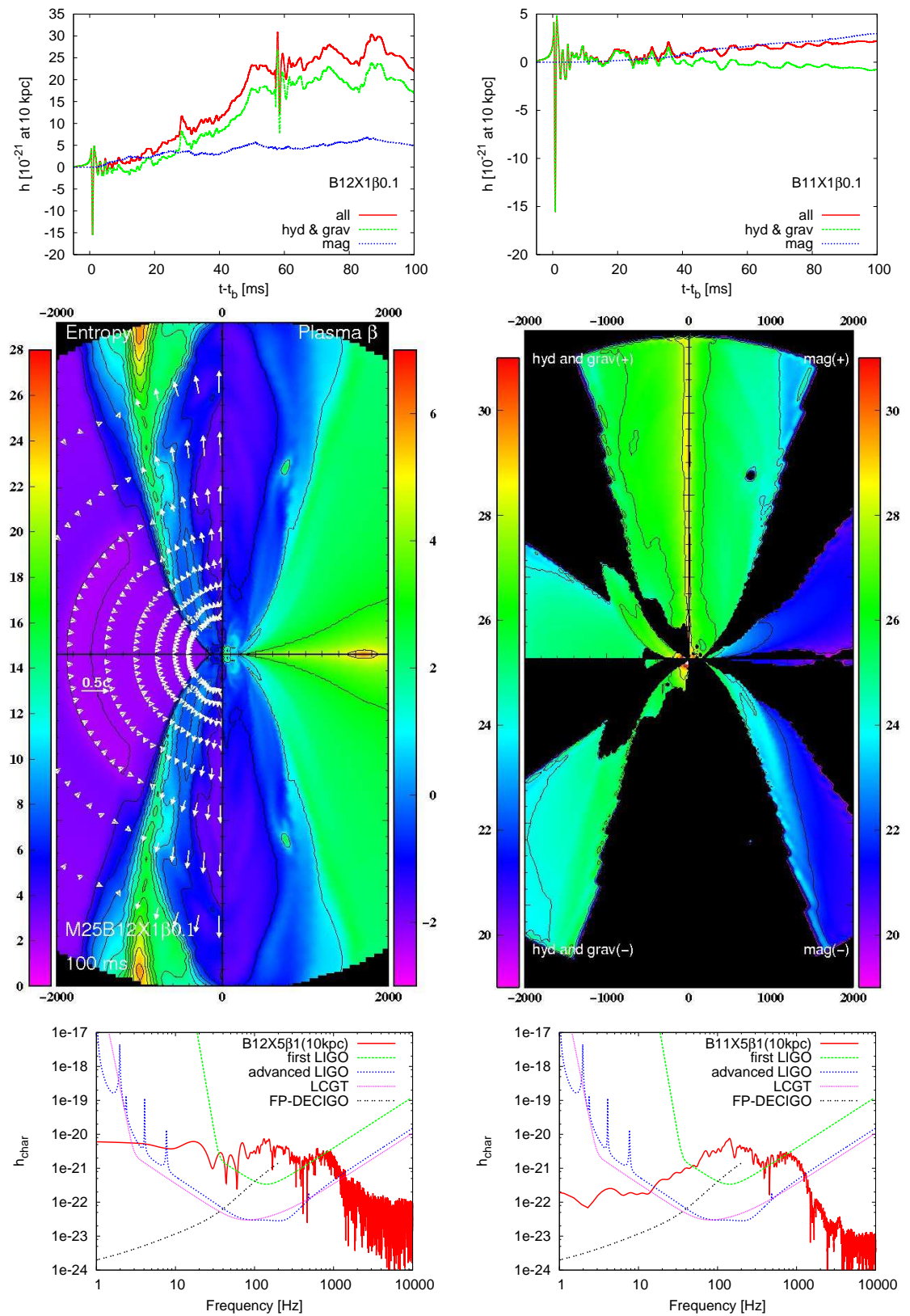

Figure 14: Top panels show examples of gravitational waveforms with the quasi-monotonically increasing trend (left) or not (right panel) obtained in the 2D SRMHD simulations in Takiwaki and Kotake (2011) [174]. Initial rotation parameter is both set to be $\beta=0.1 \%$, while the precollapse magnetic field is taken as $10^{12} \mathrm{G}$ (left) and $10^{11} \mathrm{G}$ (right), respectively. The total wave amplitudes are shown by the red line, while the contribution from the magnetic fields and from the sum of hydrodynamic and gravitational parts are shown by blue and green lines, respectively. The middle panels show various quantities for analyzing the increasing trend taking the top-left-panel model as a reference. Left panel shows the distributions of entropy $\left[k_{B} /\right.$ baryon] (left-half) and logarithm of plasma $\beta$ (right-half) at $100 \mathrm{~ms}$ after bounce. The white arrows (left-half) show the velocity fields, which are normalized by the scale in the middle left edge $(0.5 c)$. The middle right panel shows the sum of the hydrodynamic and gravitatignal parts (indicated by "hyd and grav" in the left-hand side) and the magnetic part (indicated by "mag" in the right-hand side), respectively. The top and bottom panels represent the positive and negative contribution (indicated by $(+)$ or $(-))$ to $A_{20}^{\mathrm{E} 2}$, respectively (see text for more details). The side length of the middle panels is $4000(\mathrm{~km}) \times 8000(\mathrm{~km})$. The bottom panels indicate the detectability of the GW spectra for a model with (left) or without (right) the increasing trend, respectively. The supernova is assumed to be located at the distance of $10 \mathrm{kpc}$. 
space interferometers like Fabry-Perot type DECIGO is designed to be sensitive in the frequency regimes [148, 176] (e.g., the black line in the bottom panels). These low-frequency signals, if observed, could be one important messenger of the increase-type waveforms that are likely to be associated with MHD explosions exceeding $10^{51} \mathrm{erg}$.

Concerning the microphysical aspects, the MHD simulations presented in this section are far behind the most up-to-date simulations in which the spectral neutrino transport is solved (e.g., section 2 and Table 1). This means that the GW predictions in the MHD explosions are also still in their infancy. One may easily guess that it is numerically challenging to solve the neutrino transport in the highly non-spherical environments that are inherent to MHD explosions. The PNS is deformed to be like a dumbbell and the regions outside the PNS become highly anisotropic such as by the formation of the polar funnel region after the bipolar flows pass by 27 . It is an urgent task to sophisticate the GW predictions in the MHD explosions in the same level as those in the neutrino-driven explosion models.

\subsubsection{Non-axisymmetric instabilities}

As the textbook by Chandrasekhar (1969) [177] says (see also [178, 24]), rapidly rotating compact objects can be subject to non-axisymmetric rotational instabilities when the ratio of rotational to gravitational potential energy $(T /|W|=\beta)$ exceeds a certain critical value. Since the growing instabilities carry the object's spheroidal into a triaxial configuration with a timedependent quadrupole(or higher) moment, strong GW emission can be expected (see Fryer and New (2011) [23] for a detailed review of the uncovered topics in this article).

Probably the best understood type of instability is the classical dynamical bar-mode instability with a threshold of $\beta_{\text {bar }} \gtrsim 0.27$. Rampp et al. (1998) [179] reported the first 3D hydrodynamic core-collapse simulations to study the growth of the bar mode instability and their impact on the GW emission. The initial condition for their study was based on the configuration at several milliseconds before core bounce in the rapidly rotating 2D models of Zwerger et al. (1996) [180]. In addition to the configuration, they imposed low mode $(m=3)$ density perturbation and followed the growth of the instability, where $m$ stands for the azimuthal quantum number. They observed the three clumps merged into a bar-like structure due to the growth of the non-axisymmetric instability. In fact, their models are rapid rotators whose values of $T /|W|$ exceeds the critical value beyond $\beta_{\text {bar }}$, beyond which MacLaurin spheroids become dynamically unstable again triaxial perturbations. However, they found that the maximum GW amplitudes were only $~ 2 \%$ different from the 2D cases by Zwerger et al [180]. 3D SPH simulations of a rapidly rotating model with the initial value of $T /|W|_{\text {init }}$ of $\sim 3 \%$ by Fryer and his collaborators [56, 181] reached a similar conclusion. Full GR 3D simulations by Shibata and his collaborators, on the other hand, pointed out that the maximum GW amplitudes for models with rapid rotation $\left(1 \lesssim T /|W|_{\text {init }} \lesssim 3 \%\right)$ can be enhanced by a factor of 10 than the ones predicted in the Newtonian studies mentioned above [182]. They discussed that the enhancement of the self-gravity due to the GR effects results in a more efficient spin-up of the core thus leading to the growth of the non-axisymmetric instability. The key question is whether such a precollapse rapid rotation $\left(T /|W|_{\text {init }} \gtrsim 1 \%\right)$ can be realized or not.

More recently, Ott et al. (2007) [122, 183] and Dimmelmeier et al. (2008)[110] reported a

${ }^{27}$ In such a case, the ray-by-ray approximation would be not good. Instead, the MGFLD scheme or a recently proposed M1 closure technique [166] would be much better. 
systematic study in which a wide variety of the state-of-the-art stellar evolution model 28 was employed in their 3D full GR and 2D CFC core-collapse simulations. Their results presented a strong evidence that the postbounce core, even in their extreme models, do not reach values of $\beta$ close to $\beta_{\text {dyn }}$ during collapse, bounce and during early postbounce time. The PNS rotation could reach $\beta_{\text {dyn }}$ if it would keep gravitationally contracting with conserving its angular momentum in their cooling phase (lasting on a timescale of $\sim$ minutes). However in this case, the threshold of the secular bar-mode instability $\left(\beta_{\mathrm{sec}} \sim 0.14\right)$ may be first satisfied (e.g., [184]). For more indepth reviews about this topic with their potential GW emission mechanisms, see recent reviews of Andersson (2003) [19] and Kokkotas (2008) [185].

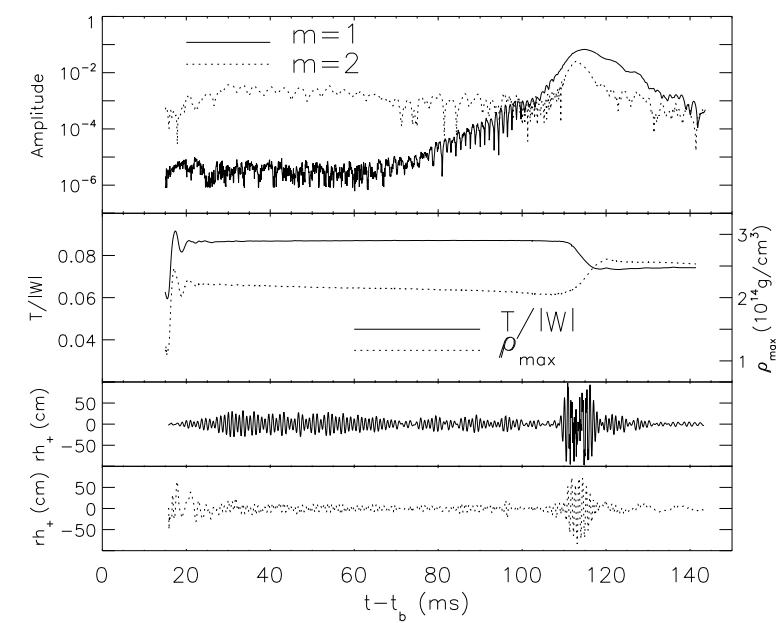

Figure 15: Time evolution of various quantities in a 3D model with $T /|W|_{\text {init }}=0.2 \%$ calculated by Ott et al. (2005) [186]. Time is measured from the epoch of core bounce $t_{b}$. Top panel shows that the amplitude of the $m=1$ mode precedes that of the $m=2$. Middle panel shows the time evolution of $T /|W|$ and the core's maximum density. It is shown from the panel that after the epoch of $t-t_{b} \sim 100 \mathrm{~ms}$, when the $m=2$ mode begins to be amplified, the transfer of the angular momentum becomes active which results in the increase of the maximum density and the decrease of the $T /|W|$. The bottom panel shows the gravitational strain at the distance to the source $r$ as viewed down the rotational axis (solid curve) and as viewed along the equatorial plane (dotted curve). One can see that the waveform traces the time evolution of the $m=2$ mode. Note in the panel that $r h=100 \mathrm{~cm}$ corresponds to $h \sim 3 \times 10^{-21}$ for a galactic supernova. This plot is by courtesy of Ott and the coauthors.

In contrast to the high $T /|W|$ instabilities mentioned above, recent work, some of which has been carried out in idealized setups and assumptions [187, 188, 189, 190, 191] and later also in more self-consistent core-collapse simulations [186, 111, 112], suggest that a differentially rotating PNS can become dynamically unstable at much lower $T /|W|$ as low as $\lesssim 0.1$. Despite clear numerical evidence for their existence, the physical origin of the low- $T /|W|$ instability remains unclear. However it has been suggested [189, 192] that the instabilities are associated with the existence of corotation points (where the pattern speed of the unstable modes matches the local angular velocity) inside the star and are thus likely to be a subclass of shear instabilitie 29 .

\footnotetext{
${ }^{28}$ also by changing a huge parameter space spanned by initial rotation rate, degree of differential rotation, and different EOSs.

${ }^{29}$ Note that corotation resonance has been long known to the key ingredients in the accretion disk system, such as the Papaloizou-Pringle instability [193].
} 
Figure 15 shows various quantities for a non-magnetized $20 M_{\odot}$ model of Ott et al. (2005) that experiences the low- $T /|W|$ instability. They investigated the growth of the non-axisymmetric structure until the rather later phases $(\geq 100 \mathrm{msec})$ after bounce [186]. They found that the growth of the $m=1$ mode, the so-called one-armed instability [194, 187, 188, 192], precedes the growth of the bar-mode $(m=2)$ instability (see the top panel in Figure 15), where $m$ denotes the azimuthal quantum number. They pointed out that the initial rotation rate can be as small as $T /|W|_{\text {init }}=0.2 \%$ for the sufficient gravitational radiation enough to be detected by the future detectors. This value is indeed much smaller than the one previously assumed for igniting the growth of the classical bar-mode instability.

More recently, Scheidegger et al. (2008,2010) [111, 112] investigated this issue by more realistic 3D MHD models that followed consistently the dynamics from core-collapse, through bounce, up to $\sim 200 \mathrm{~ms}$ postbounce in which a treatment for neutrino transport based on a partial implementation of the IDSA scheme $90-30$ was incorporated. Top panels in Figure 16$]$ show the vorticity distribution in the equatorial plane selected from their 3D MHD models, in which the major difference originates from the inclusion of the neutrino transport effect (right) or not (left). Regardless of the model difference, a two-armed $(m=2)$ pattern is clearly seen (at $\sim 10 \mathrm{~ms}$ after bounce for the plot) and then the spiral flows develop for more than several hundreds milliseconds later on. The middle panels display the quadrupole amplitude of the $A_{+}$and $A_{\times}$along the pole for their 3D MHD model with (right) or without the neutrino cooling (left). The most striking feature is that the model with neutrino cooling (right) shows $5 \sim 10 \times$ bigger maximum GW amplitudes due to the nonaxisymmetric dynamics compared to their counterparts that neglect neutrino cooling (left). They pointed out that the neutrino cooling in the postbounce phase leads to a more condensed PNS with a shorter dynamical timescale and also with much more matter enclosed in the unstable region (see the compactness of the spiral flows in the top right compared to the top left panel in Figure [16), leading to much more powerful GW. Another important message from the middle panels is that the gravitational waveforms from the nonaxisymmetric dynamics generally show narrow-band and highly quasi-periodic signals which persist until the end of simulations. For the periodic signals, the effectively measured $\mathrm{GW}$ amplitude scales with the number of GW cycles $N$ as $h_{\text {eff }} \propto h \sqrt{N}$. This is the reason why the peak amplitude in the GW spectrum (the bottom panel in Figure 16) exceed $10^{-20}$ for the galactic source, making the chance of detection quite higher. In fact, the wave amplitude from the short-duration bursts near bounce is generally smaller than $10^{-20}$ for the galactic source (e.g., the amplitude near $1 \mathrm{kHz}$ in the bottom panels in Figure 17).

For detecting (potentially) the most powerful GW signals, it is therefore of crucial importance to understand the properties of the non-axisymmetic instabilities. However the numerical difficulty to follow a long-term postbounce evolution in $3 \mathrm{D}$ is the main hindrance at present. While the angular momentum is continuously brought in the iron core from stellar envelope that rotates with higher angular momentum, the shear energy in the vicinity of the corotation points will continue to be redistributed or even dissipated by some mechanisms including the MRI. In the mentioned 3D simulations, neutrino heating is not taken into account. Neutrino-driven convection would affect the growth of the non-axisymmetric instabilities as well as through the spiral SASI modes. The general relativity that has been mostly treated in a post-Newtonian manner should affect the growth rate. At first, we could start to address this question by performing parametric explosion models in 3D MHD (e.g., sections 3.1.1 and 3.1.2). But for a more quantitative

\footnotetext{
${ }^{30}$ see section 2 for more details of the scheme.
} 

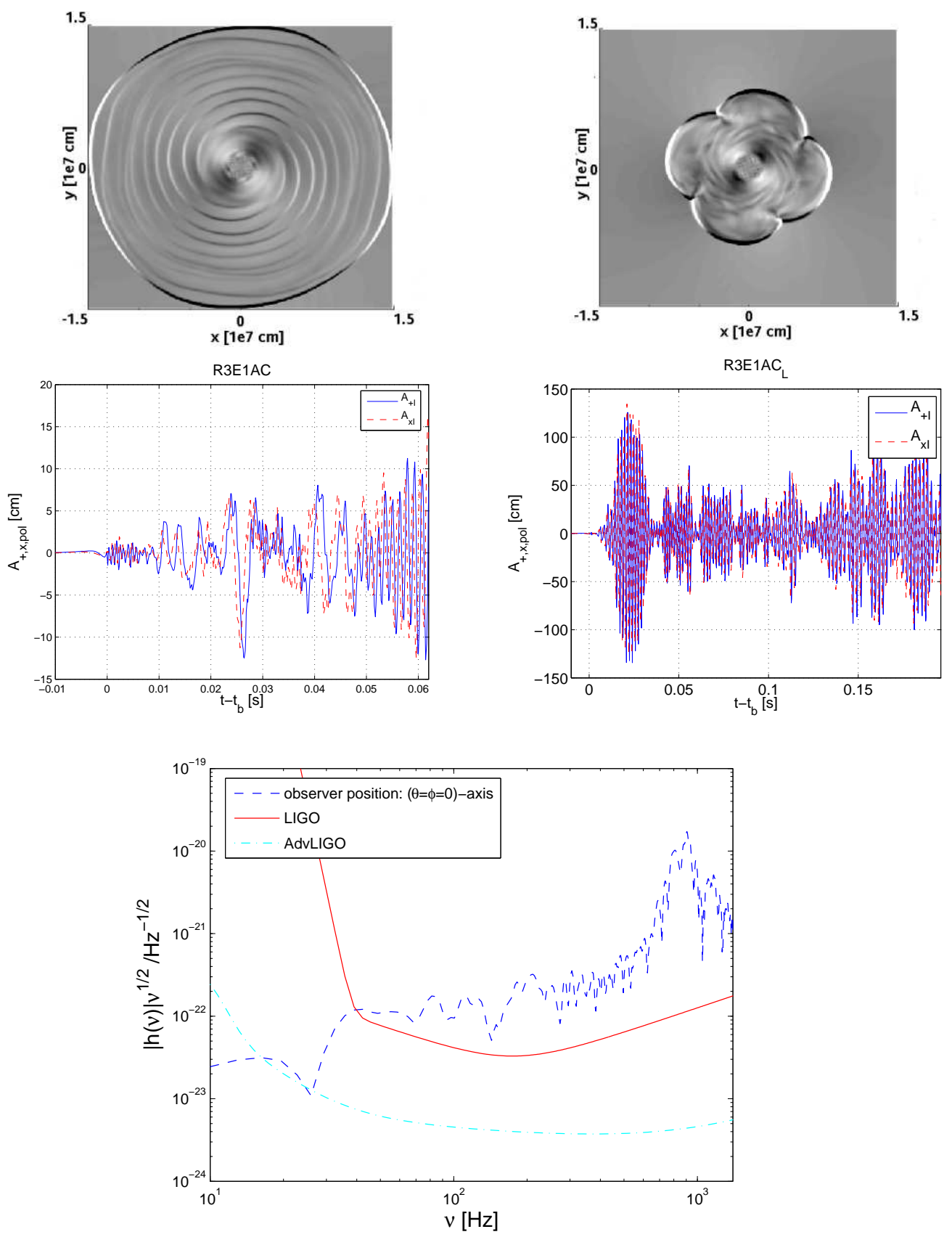

Figure 16: Top panels depict the vorticity distribution in the equatorial plane of the 3D MHD models of R4STAC (left) and R4E1FC $L$ (right) in Scheidegger et al. (2010) [112] (at $\sim 10 \mathrm{~ms}$ after bounce). A two-armed $m=2$ pattern behind the stalled shock is clearly seen (the side length of the plot is $(300 \mathrm{~km})^{2}$ ) either for their 3D model without (top left) or with the deleptonization effect (top right). The middle panels display the quadrupole amplitude of the $A_{+}$and $A_{\times}$along the pole for a given 3D MHD model without (left) or with the deleptonization effect (right). The bottom panel shows spectral energy distributions of the GW signal emitted along the polar axis for their representative 3D model experiencing the low- $T /|W|$ instability (model R4STCA) at a distance of $10 \mathrm{kpc}$. These plots are by courtesy of Scheidegger and the coauthors. 
discussion, the full 3D GRMHD simulation with an appropriate neutrino transport is needed also in this case, towards which the supernova modelers will keep their efforts (as they have done since time immemorial) and more recently people in the numerical relativity are also joining in the efforts.

\section{Summary and Concluding remarks}

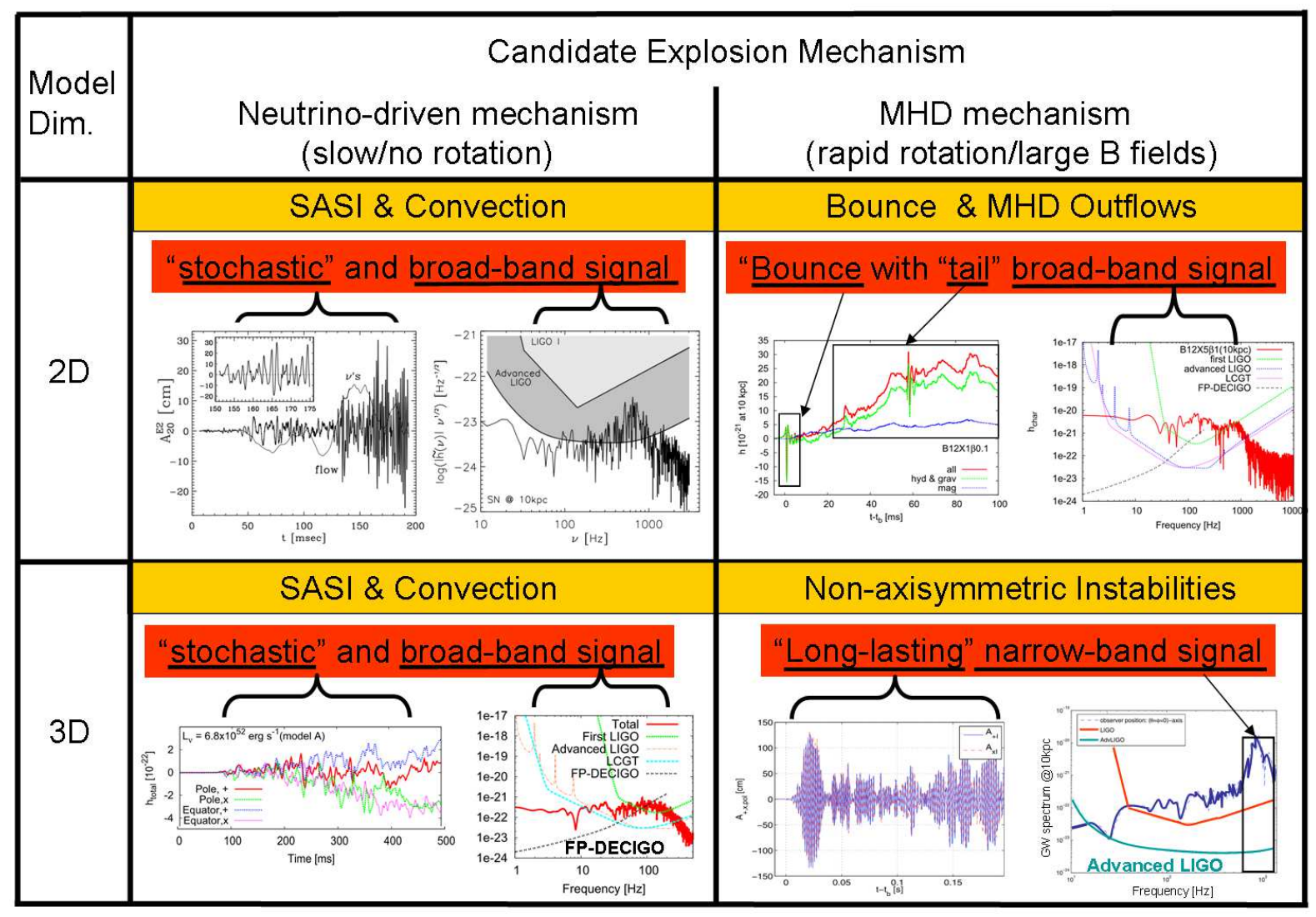

Table 3: Illustrative summarizing the relation of the potential explosion mechanisms (horizontal column) and their emission processes (columns colored by yellow) and GW signatures (columns colored by red) obtained so far in "2D" and "3D" simulations (vertical direction). In each column, the waveform (left) and the GW spectrum (right) are shown for some representative models, in which top left (2D, neutrino mechanism), top right (2D, MHD mechanism), bottom left (3D, neutrino mechanism), and bottom right (3D, MHD mechanism) are again taken from Figures $4,14,8$ and 16 (see each figure and their caption for more details). Figures in the top left and bottom right columns are taken from the results by Müller et al. (2004) [120] and Scheidegger et al. (2010) [112] by their courtesy.

The aim of writing this article was to provide an overview of what we currently know about the possible GW signatures of core-collapse SNe predicted by a number of extensive numerical 
simulations especially in the last $\sim 15$ years. In addition to the long-studied bounce GW signals, multiple new physical inputs have been proposed mostly in the last decade. Among them, we focused on the GW signatures produced by prompt convection, non-radial flows inside the PNS and in the neutrino-driven bubble, the activity of the SASI, and by asymmetric neutrino emission in the context of the neutrino-driven explosion models in section 3.1 and by asymmetries associated with the effects of magnetic fields and non-axisymmetric rotational instabilities in section 3.2. As mentioned in section 2, our understanding of the physics to unravel these waveform features has been progressing in accordance with the sophistication of the numerical simulations on which SN modelers have been putting a huge effort for long.

We wish that from Table 3, one might see a very rough illustrative summary of this article that describes a possible association between the potential explosion mechanisms (horizontal) and the emission processes with their GW signatures (columns colored by yellow and red) obtained in the recent multi-D simulations (vertical). At one sight it is a very good news for us that there is a clear correspondence between the explosion mechanisms and their GW signatures. This means that the GW observation could provide an important probe into the potential explosion mechanisms (see also Ott (2009) [22] 31. Although the criterion that bifurcates between the neutrino-driven mechanism and the MHD mechanism is still gray, rotation and magnetic fields in the precollapse cores should hold the key importance.

In the neutrino heating mechanism, the whole story may be summarized as follows. If a precollapse iron core has a "canonical" rotation rate as predicted by stellar evolution calculations $\left(\Omega_{0} \lesssim 0.1 \mathrm{rad} / \mathrm{s}\right)$, the collapse-dynamics before bounce proceeds spherically and the structures interior to the PNS are essentially spherical at this epoch. The stalling bounce shock gives rise to the GW signals of the prompt convection (see the inset of the top left panel in Table 3, and also Figure 1 in section 3.1 for more details). Typically later than $\sim 100 \mathrm{~ms}$ after bounce, convective overturns as well as the SASI in the postshock region become much more vigorous with time. Since anisotropies of the neutrino flux as well as matter motions in this epoch are governed by the non-linear hydrodynamics, the GWs signatures change also stochastically with time (sections 3.1.1 and 3.1.2). This feature was already known in the first-principle 2D simulations (indicated by "stochastic" in Table 3, e.g., section 3.1.3 for more details). Furthermore recent exploratory 3D simulations revealed that the stochastic nature becomes much more strong because the explosion geometry changes in all directions (section 3.1.4). Note that the stochastic nature could become weaker in the presence of rotation as a result of the spiral SASI (section 3.1.4, however the first-principle 3D simulations are apparently needed to draw a robust conclusion. Contributed by neutrino GWs dominant in the lower-frequency bands ( $\lesssim 100 \mathrm{~Hz}$ ), the total GW spectrum tends not to be a single peak but rather flat over a broad frequency up to $\sim 1 \mathrm{kHz}$. For detecting these GW signals for a Galactic supernova source with a good signal-to-noise ratio, we need next-generation detectors such as the advanced LIGO, LCGT, and the Fabry-Perot type DECIGO (e.g., the left panels in Table 3 (right-hand)). As a side remark, neutrino signals at this non-linear phase change also stochastically with time, which could be detectable for a Galactic supernova in a currently running detector such as by IceCube [87, 128, 195]. When material behind the stalled shock successfully absorbs enough neutrino energy to be gravitationally unbound from the iron core, a powerful neutrino-driven explosion with its explosion energy exceeding $10^{51} \mathrm{erg}$ will be obtained (section 2). In this case, the GW waveforms would imprint

\footnotetext{
${ }^{31}$ It should be also remembered that galactic core-collapse SN event is a quite rare event happening at a rate of one in a few decades. As theoreticians, we sincerely hope to be able to make clear the supernova mechanism and the related issues before observations tell us!
} 
the information of the blast morphologies (e.g., section 3.1.1).

If a precollapse core rotates enough rapidly (typically initial rotation period less than $\sim 4 \mathrm{~s}$ ) with strong magnetic fields (higher than $\sim 10^{11} \mathrm{G}$ ) imposed initially, the MHD mechanism can produce bipolar explosions along the rotational axis predominantly by the field wrapping mechanism (section 3.2 32 . The GW signals in energetic MHD explosions exceeding $10^{51} \mathrm{erg}$, are characterized by a burst-like bounce signal plus a secularly growing tail (indicated by "Bounce" with "tail" signals in the top right panel in Table 3, also section 3.2). Likewise the neutrino GWs in the case of neutrino-driven explosions, the tail component makes the total GW spectrum broad-band. Going beyond 2D, recent 3D MHD simulations show a strong evidence that nonaxisymmetric rotational instabilities can be a source of strong $\mathrm{GW}$ emission due to its periodicity and bar-mode character (see a sharp peak near $\sim 1 \mathrm{kHz}$ in the bottom right panel in Table 3 ). Note that the pole to equator anisotropy of the shock propagation in the MHD explosions could lead to a sudden decrease in the SuperKamiokande events through the MSW flavor conversion ( 2500 events for a Galactic source). A planned joint analysis of neutrino and GW data [196] would provide a powerful probe especially into the MHD-driven explosions that imprint these peculiar signatures distinct from other candidate explosion mechanisms. Note also that MHD explosions could be a possible $r$-process cite because the mass ejection from the iron core takes place in much shorter timescales than in the delayed neutrino-heating mechanism, which prevents the ejecta from being proton-rich by neutrino capture reactions [197, 198]33. As mentioned in the final part of section 3.2 these interesting possibilities have been proposed so far by the numerical simulations with a crude treatment of neutrino transport, so that they are now awaited to be re-examined.

One important notice here is that the explosion energies obtained in some of the first-principle 2D simulations (Table 1 in section 2) are underpowered by one or two orders of magnitudes to explain the canonical supernova kinetic energy $\left(\sim 10^{51} \mathrm{erg}\right)$. Moreover, the softer nuclear EOS, such as of the Lattimer-Swesty (LS) [201] EOS with an incompressibility at nuclear densities, $K$, of $180 \mathrm{MeV}$, has been employed in all the 2D simulations that succeeded in producing neutrinodriven explosion 34 . On top of a striking evidence that favors a stiffer EOS based on the nuclear experimental data $(K=240 \pm 20 \mathrm{MeV}$, [202]), the soft EOS may not account for the recently observed massive neutron star of $\sim 2 M_{\odot}$ [203] [see the maximum mass for the LS180 EOS in 204, 205]. What is then missing furthermore? To seek the answer is equal to making the GW prediction more accurate. We may get the answer by going to the first-principle 3D simulations or by taking into account new ingredients, such as exotic physics in the core of the protoneutron star [76, 206], viscous heating by the magnetorotational instability [207, 208], or energy dissipation via Alfvén waves [209]. General relativity (GR) so far treated in a very approximate way in multi-D models might help explosions if the advantage of the GR (i.e., higher neutrino energies) dominates over the disadvantage (i.e., shorter advection timescale). 3D effects might help neutrino-driven explosions as have been recently pointed out by 3D simulations with a spectral neutrino transport [79]. Assisted by a growing computer power, all these issues will be tested soon by the forth-coming first-principle simulations.

\footnotetext{
${ }^{32}$ If the MRI can be sufficiently resolved in global simulations, MHD outflows might be produced even for more weakly magnetized cores because the MRI can exponentially amplify the initial magnetic fields to a dynamically important strength within several rotational periods (e.g., collective references in Obergaulinger et al. (2009) [171]).

${ }^{33}$ See collective references in [199, 200] for other plausible $r$-process cites.

${ }^{34} 2 \mathrm{D}$ explosion was obtained in the MPA simulations for a non-rotating $11.2 M_{\odot}$ for the Shen EOS $(K=281 \mathrm{MeV})$ (H-T. Janka, private communication).
} 
In addition to these advances in numerical simulations, the physical understanding of fundamental problems in the supernova theory is progressing at the same pace. What determines the saturation levels of SASI? A careful analysis on the parasitic instabilities has been reported to answer this question [210]. What determines mode couplings between the small-scale convection eddies and the large scale SASI modes? To apply the theory of turbulence [211] might put a milestone to address this question. The physical understanding of these phenomena should also give us a deep understanding of the GW features from the SASI and convection. Although it is also numerically challenging to resolve the small scale MRI modes in global simulations, it is very interesting to see their outcome in the neutrino-driven mechanism with rapid rotation. Besides the two representative EOSs of LS and Shen, new sets of EOSs have been recently reported [95, 96, 97]. Can we extract the information of the different EOSs by the GW observation? All of these questions may not be easy to answer immediately, but they should indeed provide us a precious chance to solve them if not for the GW astronomy.

If the neutrino-heating mechanism (or some other mechanisms) fails to explode massive stars, central PNSs collapse to BHs. Recent GR simulations [212] indicate that the significant GW emission is associated at the moment of the $\mathrm{BH}$ formation, which can be a promising target of the advanced LIGO for a Galactic source. As pointed out in [213], disappearing neutrino signals could also tell us the epoch of BH formations for a Galactic source. When quarks and pions could appear in a collapsing core, one may observe a neutrino burst induced by the recollapse of the PNS, which is triggered by the sudden softening of the EOS [76]. The intervals between bounce and the BH formation depend on the details of exotic physics in the super-dense core [214, 215]. All of these observational signatures could be an important probe into the so-called dense QCD region in the QCD phase diagram (e.g., [216] for recent review) to which lattice calculations are hardly accessible at present.

Finally, what is about the story if the MHD mechanism fails ? The central cores with significant angular momentum would collapse into a $\mathrm{BH}$. Neutrinos emitted from the accretion disk heat matter in the polar funnel region [217, 218] or strong magnetic fields in the cores of order of $10^{15}$ $\mathrm{G}$ play also an active role both for driving the magneto-driven jets and for extracting a significant amount of energy from the BH (e.g., [33, 219, 220] and see references therein). This picture, often referred as collapsar [221, 222, 223], has been the working hypothesis as a central engine of long-duration gamma-ray bursts for these 20 years (see references in [224, 219, 220, 225, 226] for other candidate mechanism including magnetar models). It is indeed a big issue whether the formation of outflows predominantly proceeds via the neutrino-heating mechanism or MHD processes (see collective references in [227, 228, 92] for example). Comparing to various findings illustrated in Table 1, much little things are known about the BH-forming supernovae and about the collapsar. This may be partly because the requirement for the numerical modeling to this end is highly computationally expensive, which necessitates not only GRMHD simulations for handling the $\mathrm{BH}$ formation, but also multi-angle neutrino transfer for treating highly anisotropic neutrino radiation from the accretion disks.

To get a unified picture of massive stellar death in view of GW astronomy, we need to be able to draw a schematic picture (like Table 3) also in the case of the BH forming supernovae. A documentary film recording our endeavours to make our dream of "GW astronomy of massive stellar core-collapse" come true, seems not to come to an end immediately and is becoming even longer by taking into account the $\mathrm{BH}$ forming supernovae. In addition, a new horizon is now being opening up towards a multi-messenger astronomy by which we could have much more information to decipher the central engines of massive stars from the combined analysis between GWs, neutrinos, and electromagnetic messengers. For example, optical observations 
using a large-aperture telescope such as the Subaru telescope with a detailed spectropolarimetric technique (e.g., [28]) is expected to give us an indication of the explosion geometry, which could be also helpful to constrain the asphericity of the central engines. Needless to say, it is of crucial importance to accurately determine nucleosynthesis in the SN ejecta for modeling supernova light curves (see, [229, 230] for recent reviews). Note that these studies are now able to be updated by using recent results obtained in the first-principle simulations as mentioned in section 2 Here we would like to lay down our pen hoping that in the near future a great progress will be made in our understanding of a number of exciting and unsettled issues raised in this article.

Acknowledgements: The author wishes to thank his collaborators, Y. Suwa, T. Takiwaki, W. Iwakami-Nakano, M. Liebendörfer, S. Yamada, N. Ohnishi, K. Sato, M. Hashimoto, S. Harikae, N. Yasutake, N. Nishimura, K. Shaku, K. Sumiyoshi, and H. Suzuki. He would like to acknowledge helpful exchanges and/or permission to reprint figures with E. Müller, H.-T. Janka, M. Shibata, C. Fryer, C.D. Ott, M. Obergaulinger, J. Murphy, S. Scheidegger, K. Yakunin, T. Foglizzo, J. Novak, S. Ando, P. Cerdá-Durán, R. Fernández, E. O’Connor, T. Font, J.M. Ibañez, K. Nakamura, and T. Kuroda. It is also pleasure to thank K. Sato for continuing encouragements. Finally he would like to thank his family, his wife Ai and two-year-old daughter Yuu. Numerical computations in [81, 127, 174, 145] were carried out in part on XT4 and general common use computer system at the center for Computational Astrophysics, CfCA, the National Astronomical Observatory of Japan. This study was supported in part by the Grants-in-Aid for the Scientific Research from the Ministry of Education, Science and Culture of Japan (Nos. 19540309, 20740150, and 23540323) and by HPCI Strategic Program of Japanese MEXT.

\section{References}

[1] S. Ando, J. F. Beacom, H. Yüksel, Detection of Neutrinos from Supernovae in Nearby Galaxies, Physical Review Letters 95 (17) (2005) 171101-+. arXiv:arXiv:astro-ph/0503321 doi:10.1103/PhysRevLett.95.171101

[2] K. Hirata, T. Kajita, M. Koshiba, M. Nakahata, Y. Oyama, Observation of a neutrino burst from the supernova SN1987A, Physical Review Letters 58 (1987) 1490-1493. doi:10.1103/PhysRevLett.58.1490

[3] R. M. Bionta, G. Blewitt, C. B. Bratton, D. Casper, A. Ciocio, Observation of a neutrino burst in coincidence with supernova 1987A in the Large Magellanic Cloud, Physical Review Letters 58 (1987) 1494-1496. doi:10.1103/PhysRevLett.58.1494

[4] K. Sato, H. Suzuki, Analysis of the neutrino burst from supernova 1987A in the Large Magellanic Cloud, Physical Review Letters 58 (1987) 2722-2725. doi:10.1103/PhysRevLett.58.2722

[5] G. G. Raffelt, Physics with supernovae, Nuclear Physics B Proceedings Supplements 110 (2002) $254-267$. arXiv: arXiv:hep-ph/0201099 doi:10.1016/S0920-5632(02)80132-3

[6] Y. Totsuka, Neutrino astronomy, Reports on Progress in Physics 55 (1992) 377-430. doi:10.1088/0034-4885/55/3/002

[7] T. Totani, K. Sato, H. E. Dalhed, J. R. Wilson, Future Detection of Supernova Neutrino Burst and Explosion Mechanism, Astrophys. J.496 (1998) 216-+. arXiv:arXiv:astro-ph/9710203 doi:10.1086/305364

[8] J. F. Beacom, Supernovae and neutrinos, Nuclear Physics B Proceedings Supplements 118 (2003) $307-314$. arXiv:arXiv: astro-ph/0209136 doi:10.1016/S0920-5632(03)01323-9

[9] G. G. Raffelt, Physics opportunities with supernova neutrinos, Progress in Particle and Nuclear Physics 64 (2010) 393-399. doi:10.1016/j.ppnp.2009.12.057

[10] M. Abbott et al., Upper limits from the LIGO and TAMA detectors on the rate of gravitational-wave bursts, Phys. Rev. D72 (12) (2005) 122004-+. arXiv:arXiv:gr-qc/0507081 doi:10.1103/PhysRevD.72.122004

[11] M. Ando, the TAMA Collaboration, Current status of the TAMA300 gravitational-wave detector, Classical and Quantum Gravity 22 (2005) 881-+. doi:10.1088/0264-9381/22/18/S02

[12] J. Hough, S. Rowan, B. S. Sathyaprakash, The search for gravitational waves, Journal of Physics B Atomic Molecular Physics 38 (2005) 497-+. arXiv:arXiv:gr-qc/0501007, doi:10.1088/0953-4075/38/9/004

[13] M. van der Sluys, Gravitational waves from compact binaries, ArXiv e-printsarXiv:1108.1307 
[14] G. M. Harry, the LIGO Scientific Collaboration, Advanced LIGO: the next generation of gravitational wave detectors, Classical and Quantum Gravity 27 (8) (2010) 084006-+. doi:10.1088/0264-9381/27/8/084006

[15] K. Kuroda, LCGT Collaboration, Status of LCGT, Classical and Quantum Gravity 27 (8) (2010) 084004-+. doi:10.1088/0264-9381/27/8/084004

[16] B. S. Sathyaprakash, B. F. Schutz, Physics, Astrophysics and Cosmology with Gravitational Waves, Living Reviews in Relativity 12 (2009) 2-+. arXiv:0903.0338

[17] J. Faber, Status of neutron star-black hole and binary neutron star simulations, Classical and Quantum Gravity 26 (11) (2009) 114004-+. doi:10.1088/0264-9381/26/11/114004

[18] M. D. Duez, Numerical relativity confronts compact neutron star binaries: a review and status report, Classical and Quantum Gravity 27 (11) (2010) 114002-+. arXiv:0912.3529 doi:10.1088/0264-9381/27/11/114002

[19] N. Andersson, TOPICAL REVIEW: Gravitational waves from instabilities in relativistic stars, Classical and Quantum Gravity 20 (2003) 105-+. arXiv:arXiv:astro-ph/0211057

[20] C. J. Horowitz, Multi-messenger observations of neutron rich matter, ArXiv e-printsarXiv:1106.1661

[21] K. Kotake, K. Sato, K. Takahashi, Explosion mechanism, neutrino burst and gravitational wave in core-collapse supernovae., Reports of Progress in Physics 69 (2006) 971-1143. arXiv:arXiv: astro-ph/0509456

[22] C. D. Ott, TOPICAL REVIEW: The gravitational-wave signature of core-collapse supernovae, Classical and Quantum Gravity 26 (6) (2009) 063001-+. arXiv:0809.0695 doi:10.1088/0264-9381/26/6/063001

[23] C. L. Fryer, K. C. B. New, Gravitational Waves from Gravitational Collapse, Living Reviews in Relativity 14 (2011) 1-+

[24] S. L. Shapiro, S. A. Teukolsky, Black holes, white dwarfs, and neutron stars: The physics of compact objects, Research supported by the National Science Foundation. New York, Wiley-Interscience, 1983, 663 p., 1983.

[25] L. Wang, D. A. Howell, P. Höflich, J. C. Wheeler, Bipolar Supernova Explosions, Astrophys. J. 550 (2001) 10301035. doi:10.1086/319822

[26] L. Wang, J. C. Wheeler, P. Höflich, A. Khokhlov, D. Baade, D. Branch, P. Challis, A. V. Filippenko, C. Fransson, P. Garnavich, R. P. Kirshner, P. Lundqvist, R. McCray, N. Panagia, C. S. J. Pun, M. M. Phillips, G. Sonneborn, N. B. Suntzeff, The Axisymmetric Ejecta of Supernova 1987A, Astrophys. J. 579 (2002) 671-677. doi: $10.1086 / 342824$

[27] K. Maeda, K. Kawabata, P. A. Mazzali, M. Tanaka, S. Valenti, K. Nomoto, T. Hattori, J. Deng, E. Pian, S. Taubenberger, M. Iye, T. Matheson, A. V. Filippenko, K. Aoki, G. Kosugi, Y. Ohyama, T. Sasaki, T. Takata, Asphericity in Supernova Explosions from Late-Time Spectroscopy, Science 319 (2008) 1220-. arXiv:0801.1100. doi:10.1126/science.1149437

[28] M. Tanaka, K. S. Kawabata, K. Maeda, M. Iye, T. Hattori, E. Pian, K. Nomoto, P. A. Mazzali, N. Tominaga, Spectropolarimetry of the Unique Type Ib Supernova 2005bf: Larger Asymmetry Revealed by Later-Phase Data, Astrophys. J. 699 (2009) 1119-1124. arXiv:0906.1062 doi:10.1088/0004-637X/699/2/1119

[29] K. Kjær, B. Leibundgut, C. Fransson, A. Jerkstrand, J. Spyromilio, The 3-D structure of SN 1987A's inner ejecta, Astron. Astrophys.517 (2010) A51+. arXiv: 1003.5684 doi:10.1051/0004-6361/201014538

[30] D. C. Leonard, A. V. Filippenko, M. Ganeshalingam, F. J. D. Serduke, W. Li, B. J. Swift, A. Gal-Yam, R. J. Foley, D. B. Fox, S. Park, J. L. Hoffman, D. S. Wong, A non-spherical core in the explosion of supernova SN 2004dj, Nature440 (2006) 505-507. arXiv: arXiv:astro-ph/0603297. doi:10.1038/nature04558

[31] D. C. Leonard, A. V. Filippenko, M. Ganeshalingam, F. J. D. Serduke, W. Li, B. J. Swift, A. Gal-Yam, R. J. Foley, D. B. Fox, S. Park, J. L. Hoffman, D. S. Wong, A non-spherical core in the explosion of supernova SN 2004dj, Nature440 (2006) 505-507. arXiv: astro-ph/0603297 doi:10.1038/nature04558

[32] A. M. Khokhlov, P. A. Höflich, E. S. Oran, J. C. Wheeler, L. Wang, A. Y. Chtchelkanova, Jet-induced Explosions of Core Collapse Supernovae, Astrophys. J. Lett. 524 (1999) L107-L110. doi : 10.1086/312305

[33] J. C. Wheeler, I. Yi, P. Höflich, L. Wang, Asymmetric Supernovae, Pulsars, Magnetars, and Gamma-Ray Bursts, Astrophys. J. 537 (2000) 810-823. arXiv:arXiv:astro-ph/9909293. doi:10.1086/309055

[34] S. P. Mikheev, A. I. Smirnov, Resonant amplification of neutrino oscillations in matter and solar-neutrino spectroscopy, Nuovo Cimento C Geophysics Space Physics C 9 (1986) 17-26. doi : 10.1007/BF02508049

[35] H. Duan, J. P. Kneller, TOPICAL REVIEW: Neutrino flavour transformation in supernovae, Journal of Physics G Nuclear Physics 36 (11) (2009) 113201-+. arXiv:0904.0974 doi:10.1088/0954-3899/36/11/113201

[36] H.-T. Janka, K. Langanke, A. Marek, G. Martínez-Pinedo, B. Müller, Theory of core-collapse supernovae, Phys. Rep.442 (2007) 38-74. arXiv:arXiv:astro-ph/0612072 doi:10.1016/j.physrep.2007.02.002

[37] A. Burrows, L. Dessart, C. D. Ott, E. Livne, Multi-dimensional explorations in supernova theory, Phys. Rep.442 (2007) 23-37. arXiv:arXiv:astro-ph/0612460 doi:10.1016/j.physrep.2007.02.001

[38] E. Chassande-Mottin, M. Hendry, P. J. Sutton, S. Márka, Multimessenger astronomy with the Einstein Telescope, General Relativity and Gravitation 43 (2011) 437-464. arXiv:1004.1964 doi:10.1007/s10714-010-1019-z

[39] S. Márka, for the LIGO Scientific Collaboration, the Virgo Collaboration, Transient multimessenger astronomy with gravitational waves, Classical and Quantum Gravity 28 (11) (2011) 114013-+. 
doi:10.1088/0264-9381/28/11/114013

[40] T. Pradier, Antares Collaboration, The Antares neutrino telescope and multi-messenger astronomy, Classical and Quantum Gravity 27 (19) (2010) 194004-+. arXiv:1004.5579 doi:10.1088/0264-9381/27/19/194004

[41] Y. Aso, Z. Márka, C. Finley, J. Dwyer, K. Kotake, S. Márka, Search method for coincident events from LIGO and IceCube detectors, Classical and Quantum Gravity 25 (11) (2008) 114039-+. arXiv:0711.0107. doi:10.1088/0264-9381/25/11/114039

[42] S. A. Colgate, R. H. White, The Hydrodynamic Behavior of Supernovae Explosions, Astrophys. J.143 (1966) 626-+. doi:10.1086/148549

[43] J. R. Wilson, Supernovae and Post-Collapse Behavior, in: Numerical Astrophysics, 1985, pp. 422-+.

[44] H. A. Bethe, J. R. Wilson, Revival of a stalled supernova shock by neutrino heating, Astrophys. J.295 (1985) 14-23. doi:10.1086/163343

[45] H. A. Bethe, Supernova mechanisms, Reviews of Modern Physics 62 (1990) 801-866. doi:10.1103/RevModPhys.62.801

[46] J. Nordhaus, A. Burrows, A. Almgren, J. Bell, Dimension as a Key to the Neutrino Mechanism of Core-collapse Supernova Explosions, Astrophys. J.720 (2010) 694-703. arXiv:1006.3792. doi: $10.1088 / 0004-637 \mathrm{X} / 720 / 1 / 694$

[47] M. Rampp, H.-T. Janka, Spherically Symmetric Simulation with Boltzmann Neutrino Transport of Core Collapse and Postbounce Evolution of a 15 Msun Star, Astrophys. J. Lett. 539 (2000) L33-L36.

[48] M. Liebendörfer, A. Mezzacappa, F. Thielemann, Conservative general relativistic radiation hydrodynamics in spherical symmetry and comoving coordinates, Phys. Rev. D63 (10) (2001) 104003-+.

[49] T. A. Thompson, A. Burrows, P. A. Pinto, Shock Breakout in Core-Collapse Supernovae and Its Neutrino Signature, Astrophys. J. 592 (2003) 434-456.

[50] K. Sumiyoshi, S. Yamada, H. Suzuki, H. Shen, S. Chiba, H. Toki, Postbounce Evolution of CoreCollapse Supernovae: Long-Term Effects of the Equation of State, Astrophys. J. 629 (2005) 922-932. arXiv:arXiv: astro-ph/0506620 doi:10.1086/431788

[51] F. S. Kitaura, H.-T. Janka, W. Hillebrandt, Explosions of O-Ne-Mg cores, the Crab supernova, and subluminous type II-P supernovae, Astron. Astrophys.450 (2006) 345-350. arXiv:arXiv:astro-ph/0512065 doi:10.1051/0004-6361:20054703

[52] M. Herant, W. Benz, W. R. Hix, C. L. Fryer, S. A. Colgate, Inside the supernova: A powerful convective engine, Astrophys. J.435 (1994) 339-361. arXiv:arXiv:astro-ph/9404024 doi:10.1086/174817

[53] A. Burrows, J. Hayes, B. A. Fryxell, On the Nature of Core-Collapse Supernova Explosions, Astrophys. J.450 (1995) 830-+. arXiv: arXiv:astro-ph/9506061 doi:10.1086/176188

[54] H. Janka, E. Müller, Neutrino heating, convection, and the mechanism of Type-II supernova explosions., Astron. Astrophys.306 (1996) 167-+.

[55] C. L. Fryer, D. E. Holz, S. A. Hughes, Gravitational Wave Emission from Core Collapse of Massive Stars, Astrophys. J. 565 (2002) 430-446.

[56] C. L. Fryer, Neutron Star Kicks from Asymmetric Collapse, Astrophys. J. Lett. 601 (2004) L175-L178.

[57] J. M. Blondin, A. Mezzacappa, C. DeMarino, Stability of Standing Accretion Shocks, with an Eye toward CoreCollapse Supernovae, Astrophys. J. 584 (2003) 971-980. arXiv: astro-ph/0210634 doi:10.1086/345812

[58] L. Scheck, T. Plewa, H.-T. Janka, K. Kifonidis, E. Müller, Pulsar Recoil by Large-Scale Anisotropies in Supernova Explosions, Physical Review Letters 92 (1) (2004) 011103-+. arXiv : astro-ph/0307352

[59] L. Scheck, K. Kifonidis, H. Janka, E. Müller, Multidimensional supernova simulations with approximative neutrino transport. I. Neutron star kicks and the anisotropy of neutrino-driven explosions in two spatial dimensions, Astron. Astrophys.457 (2006) 963-986. arXiv:arXiv:astro-ph/0601302. doi:10.1051/0004-6361:20064855

[60] N. Ohnishi, K. Kotake, S. Yamada, Numerical Analysis of Standing Accretion Shock Instability with Neutrino Heating in Supernova Cores, Astrophys. J. 641 (2006) 1018-1028. arXiv:astro-ph/0509765 doi: $10.1086 / 500554$

[61] N. Ohnishi, K. Kotake, S. Yamada, Inelastic Neutrino-Helium Scatterings and Standing Accretion Shock Instability in Core-Collapse Supernovae, Astrophys. J.667 (2007) 375-381. arXiv:arXiv:astro-ph/0606187 doi: $10.1086 / 520755$

[62] T. Foglizzo, L. Scheck, H.-T. Janka, Neutrino-driven Convection versus Advection in Core-Collapse Supernovae, Astrophys. J.652 (2006) 1436-1450. arXiv: arXiv: astro-ph/0507636 doi:10.1086/508443

[63] J. W. Murphy, A. Burrows, Criteria for Core-Collapse Supernova Explosions by the Neutrino Mechanism, Astrophys. J.688 (2008) 1159-1175. arXiv:0805.3345, doi:10.1086/592214

[64] R. Fernández, C. Thompson, Stability of a Spherical Accretion Shock with Nuclear Dissociation, Astrophys. J.697 (2009) 1827-1841. arXiv:0811.2795 doi:10.1088/0004-637X/697/2/1827

[65] R. Fernández, C. Thompson, Dynamics of a Spherical Accretion Shock with Neutrino Heating and Alpha-Particle Recombination, Astrophys. J.703 (2009) 1464-1485. arXiv:0812.4574 
doi:10.1088/0004-637X/703/2/1464

[66] W. Iwakami, K. Kotake, N. Ohnishi, S. Yamada, K. Sawada, Three-Dimensional Simulations of Standing Accretion Shock Instability in Core-Collapse Supernovae, Astrophys. J. 678 (2008) 1207-1222. arXiv:0710.2191. doi: $10.1086 / 533582$

[67] W. Iwakami, K. Kotake, N. Ohnishi, S. Yamada, K. Sawada, Effects of Rotation on Standing Accretion Shock Instability in Nonlinear Phase for Core-Collapse Supernovae, Astrophys. J. 700 (2009) 232-242. arXiv:0811.0651 doi:10.1088/0004-637X/700/1/232

[68] R. Fernández, The Spiral Modes of the Standing Accretion Shock Instability, Astrophys. J.725 (2010) 1563-1580. arXiv: 1003.1730 doi:10.1088/0004-637X/725/2/1563

[69] H.-T. Janka, Conditions for shock revival by neutrino heating in core-collapse supernovae, Astron. Astrophys.368 (2001) 527-560. arXiv: arXiv: astro-ph/0008432 doi:10.1051/0004-6361:20010012

[70] A. Wongwathanarat, N. J. Hammer, E. Müller, An axis-free overset grid in spherical polar coordinates for simulating 3D self-gravitating flows, Astron. Astrophys.514 (2010) A48+. arXiv:1003.1633 doi:10.1051/0004-6361/200913435

[71] K. Nomoto, M. Mashimoto, Presupernova evolution of massive stars., Phys. Rep.163 (1988) 13-36. doi:10.1016/0370-1573(88)90032-4

[72] S. E. Woosley, A. Heger, T. A. Weaver, The evolution and explosion of massive stars, Reviews of Modern Physics 74 (2002) 1015-1071. doi:10.1103/RevModPhys.74.1015

[73] S. E. Woosley, T. A. Weaver, The Evolution and Explosion of Massive Stars. II. Explosive Hydrodynamics and Nucleosynthesis, Astrophys. J. Supp.101 (1995) 181-+. doi:10.1086/192237

[74] H.-T. Janka, B. Müller, F. S. Kitaura, R. Buras, Dynamics of shock propagation and nucleosynthesis conditions in O-Ne-Mg core supernovae, Astron. Astrophys.485 (2008) 199-208. arXiv:0712.4237. doi:10.1051/0004-6361:20079334

[75] A. Burrows, E. Livne, L. Dessart, C. D. Ott, J. Murphy, A New Mechanism for Core-Collapse Supernova Explosions, Astrophys. J. 640 (2006) 878-890. arXiv: astro-ph/0510687 doi:10.1086/500174

[76] I. Sagert, T. Fischer, M. Hempel, G. Pagliara, J. Schaffner-Bielich, A. Mezzacappa, F. Thielemann, M. Liebendörfer, Signals of the QCD Phase Transition in Core-Collapse Supernovae, Physical Review Letters 102 (8) (2009) 081101-+. arXiv:0809.4225 doi:10.1103/PhysRevLett.102.081101

[77] R. Buras, H.-T. Janka, M. Rampp, K. Kifonidis, Two-dimensional hydrodynamic core-collapse supernova simulations with spectral neutrino transport. II. Models for different progenitor stars, Astron. Astrophys.457 (2006) 281-308. arXiv:arXiv:astro-ph/0512189 doi:10.1051/0004-6361:20054654

[78] A. Burrows, E. Livne, L. Dessart, C. D. Ott, J. Murphy, Features of the Acoustic Mechanism of CoreCollapse Supernova Explosions, Astrophys. J. 655 (2007) 416-433. arXiv:arXiv:astro-ph/0610175 doi:10.1086/509773

[79] T. Takiwaki, K. Kotake, Y. Suwa, Three-dimensional Hydrodynamic Core-Collapse Supernova Simulations for an \$11.2 M_\{ \odot\}\$ Star with Spectral Neutrino Transport, ArXiv e-print $\operatorname{arXiv:1108.3989}$

[80] S. W. Bruenn, A. Mezzacappa, W. R. Hix, J. M. Blondin, P. Marronetti, O. E. B. Messer, C. J. Dirk, S. Yoshida, 2D and 3D Core-Collapse Supernovae Simulation Results Obtained with the CHIMERA Code, ArXiv eprints arXiv: 1002.4914

[81] Y. Suwa, K. Kotake, T. Takiwaki, S. C. Whitehouse, M. Liebendörfer, K. Sato, Explosion Geometry of a Rotating 13Msun Star Driven by the SASI-Aided Neutrino-Heating Supernova Mechanism, Publ. Astron. Soc. Japan62 (2010) L49+. arXiv:0912.1157

[82] A. Marek, H.-T. Janka, Delayed Neutrino-Driven Supernova Explosions Aided by the Standing Accretion-Shock Instability, Astrophys. J. 694 (2009) 664-696. arXiv:0708.3372 doi:10.1088/0004-637X/694/1/664

[83] C. Y. Cardall, Supernova neutrino challenges, Nuclear Physics B Proceedings Supplements 145 (2005) 295-300. arXiv:arXiv:astro-ph/0502232 doi:10.1016/j.nuclphysbps.2005.04.026

[84] A. Heger, S. E. Woosley, H. C. Spruit, Presupernova Evolution of Differentially Rotating Massive Stars Including Magnetic Fields, Astrophys. J. 626 (2005) 350-363. arXiv:arXiv:astro-ph/0409422 doi: $10.1086 / 429868$

[85] R. Buras, M. Rampp, H.-T. Janka, K. Kifonidis, Two-dimensional hydrodynamic core-collapse supernova simulations with spectral neutrino transport. I. Numerical method and results for a $15 \mathrm{Moj}$ star, Astron. Astrophys.447 (2006) 1049-1092. arXiv:arXiv:astro-ph/0507135 doi:10.1051/0004-6361:20053783

[86] C. D. Ott, A. Burrows, L. Dessart, E. Livne, Two-Dimensional Multiangle, Multigroup Neutrino Radiation-Hydrodynamic Simulations of Postbounce Supernova Cores, Astrophys. J.685 (2008) 1069-1088. arXiv:0804.0239 doi:10.1086/591440

[87] T. D. Brandt, A. Burrows, C. D. Ott, E. Livne, Results from Core-collapse Simulations with Multi-dimensional, Multi-angle Neutrino Transport, Astrophys. J.728 (2011) 8-+. arXiv:1009.4654 doi: $10.1088 / 0004-637 X / 728 / 1 / 8$

[88] S. W. Bruenn, Stellar core collapse - Numerical model and infall epoch, Astrophys. J. Supp.58 (1985) 771-841. 
doi:10.1086/191056

[89] K. N. Yakunin, P. Marronetti, A. Mezzacappa, S. W. Bruenn, C. Lee, M. A. Chertkow, W. R. Hix, J. M. Blondin, E. J. Lentz, O. E. Bronson Messer, S. Yoshida, Gravitational waves from core collapse supernovae, Classical and Quantum Gravity 27 (19) (2010) 194005-+. arXiv:1005.0779 doi:10.1088/0264-9381/27/19/194005

[90] M. Liebendörfer, S. C. Whitehouse, T. Fischer, The Isotropic Diffusion Source Approximation for Supernova Neutrino Transport, Astrophys. J.698 (2009) 1174-1190. arXiv:0711.2929. doi:10.1088/0004-637X/698/2/1174

[91] M. Liebendörfer, M. Rampp, H.-T. Janka, A. Mezzacappa, Supernova Simulations with Boltzmann Neutrino Transport: A Comparison of Methods, Astrophys. J. 620 (2005) 840-860. arXiv: arXiv: astro-ph/0310662. doi: $10.1086 / 427203$

[92] S. Harikae, K. Kotake, T. Takiwaki, Y.-i. Sekiguchi, A General Relativistic Ray-tracing Method for Estimating the Energy and Momentum Deposition by Neutrino Pair Annihilation in Collapsars, Astrophys. J.720 (2010) 614-625. doi:10.1088/0004-637X/720/1/614

[93] B. Müller, H.-T. Janka, H. Dimmelmeier, A New Multi-dimensional General Relativistic Neutrino Hydrodynamic Code for Core-collapse Supernovae. I. Method and Code Tests in Spherical Symmetry, Astrophys. J. Supp.189 (2010) 104-133. arXiv:1001.4841 doi:10.1088/0067-0049/189/1/104

[94] M. Shibata, K. Kiuchi, Y.-i. Sekiguchi, Y. Suwa, Truncated Moment Formalism for Radiation Hydrodynamics in Numerical Relativity, ArXiv e-prints $\operatorname{arXiv:1104.3937}$

[95] M. Hempel, J. Schaffner-Bielich, A statistical model for a complete supernova equation of state, Nuclear Physics A 837 (2010) 210-254. arXiv:0911.4073 doi:10.1016/j.nuclphysa.2010.02.010

[96] S. Furusawa, S. Yamada, K. Sumiyoshi, H. Suzuki, A new baryonic equation of state at sub-nuclear densities for core-collapse simulations, ArXiv e-print $\operatorname{arXiv:1103.6129}$

[97] G. Shen, C. J. Horowitz, E. O'Connor, Second relativistic mean field and virial equation of state for astrophysical simulations, Phys. Rev. C83 (6) (2011) 065808-+. arXiv:1103.5174 doi:10.1103/PhysRevC.83.065808

[98] E. Müller, Gravitational radiation from collapsing rotating stellar cores, Astron. Astrophys.114 (1982) 53-59.

[99] R. Mönchmeyer, G. Schaefer, E. Mueller, R. E. Kates, Gravitational waves from the collapse of rotating stellar cores, Astron. Astrophys. 246 (1991) 417-440.

[100] S. Yamada, K. Sato, Gravitational Radiation from Rotational Collapse of a Supernova Core, Astrophys. J.450 (1995) 245-+. doi:10.1086/176135

[101] T. Zwerger, E. Müller, Dynamics and gravitational wave signature of axisymmetric rotational core collapse., Astron. Astrophys. 320 (1997) 209-227.

[102] K. Kotake, S. Yamada, K. Sato, Gravitational radiation from axisymmetric rotational core collapse, Phys. Rev. D68 (4) (2003) 044023-+.

[103] K. Kotake, S. Yamada, K. Sato, K. Sumiyoshi, H. Ono, H. Suzuki, Gravitational radiation from rotational core collapse: Effects of magnetic fields and realistic equations of state, Phys. Rev. D69 (12) (2004) 124004-+.

[104] M. Shibata, Y.-I. Sekiguchi, Gravitational waves from axisymmetric rotating stellar core collapse to a neutron star in full general relativity, Phys. Rev. D69 (8) (2004) 084024-+. arXiv:arXiv:gr-qc/0402040 doi:10.1103/PhysRevD.69.084024

[105] C. D. Ott, A. Burrows, E. Livne, R. Walder, Gravitational Waves from Axisymmetric, Rotating Stellar Core Collapse, Astrophys. J. 600 (2004) 834-864. arXiv: arXiv:astro-ph/0307472 doi:10.1086/379822

[106] C. D. Ott, H. Dimmelmeier, A. Marek, H. Janka, I. Hawke, B. Zink, E. Schnetter, 3D Collapse of Rotating Stellar Iron Cores in General Relativity Including Deleptonization and a Nuclear Equation of State, Physical Review Letters 98 (26) (2007) 261101-+. arXiv:arXiv:astro-ph/0609819. doi:10.1103/PhysRevLett.98.261101

[107] C. D. Ott, H. Dimmelmeier, A. Marek, H. Janka, B. Zink, I. Hawke, E. Schnetter, Rotating collapse of stellar iron cores in general relativity, Classical and Quantum Gravity 24 (2007) 139-+. arXiv:arXiv:astro-ph/0612638 doi:10.1088/0264-9381/24/12/S10

[108] H. Dimmelmeier, J. A. Font, E. Müller, Relativistic simulations of rotational core collapse II. Collapse dynamics and gravitational radiation, Astron. Astrophys. 393 (2002) 523-542.

[109] H. Dimmelmeier, C. D. Ott, H.-T. Janka, A. Marek, E. Müller, Generic Gravitational-Wave Signals from the Collapse of Rotating Stellar Cores, Physical Review Letters 98 (25) (2007) 251101-+. arXiv:arXiv:astro-ph/0702305 doi:10.1103/PhysRevLett.98.251101

[110] H. Dimmelmeier, C. D. Ott, A. Marek, H. Janka, Gravitational wave burst signal from core collapse of rotating stars, Phys. Rev. D78 (6) (2008) 064056-+. arXiv:0806.4953 doi:10.1103/PhysRevD.78.064056

[111] S. Scheidegger, T. Fischer, S. C. Whitehouse, M. Liebendörfer, Gravitational waves from 3D MHD core collapse simulations, Astron. Astrophys. 490 (2008) 231-241. arXiv:0709.0168. doi:10.1051/0004-6361:20078577

[112] S. Scheidegger, R. Käppeli, S. C. Whitehouse, T. Fischer, M. Liebendörfer, The influence of model parameters on the prediction of gravitational wave signals from stellar core collapse, Astron. Astrophys.514 (2010) A51+. 
doi:10.1051/0004-6361/200913220

[113] M. Liebendörfer, A Simple Parameterization of the Consequences of Deleptonization for Simulations of Stellar Core Collapse, Astrophys. J.633 (2005) 1042-1051. arXiv:arXiv:astro-ph/0504072. doi: $10.1086 / 466517$

[114] S. E. Woosley, A. Heger, The Progenitor Stars of Gamma-Ray Bursts, Astrophys. J. 637 (2006) 914-921. arXiv:arXiv:astro-ph/0508175 doi:10.1086/498500

[115] S.-C. Yoon, N. Langer, Evolution of rapidly rotating metal-poor massive stars towards gamma-ray bursts, Astron Astrophys. 443 (2005) 643-648. arXiv: arXiv: astro-ph/0508242 doi:10.1051/0004-6361:20054030

[116] C. D. Ott, A. Burrows, T. A. Thompson, E. Livne, R. Walder, The Spin Periods and Rotational Profiles of Neutron Stars at Birth, Astrophys. J., Suppl. Ser. 164 (2006) 130-155. arXiv:arXiv:astro-ph/0508462 doi: $10.1086 / 500832$

[117] E. Müler, H.-T. Janka, Gravitational radiation from convective instabilities in Type II supernova explosions., Astron. Astrophys. 317 (1997) 140-163.

[118] A. Burrows, J. Hayes, Pulsar Recoil and Gravitational Radiation Due to Asymmetrical Stellar Collapse and Explosion, Physical Review Letters 76 (1996) 352-355. arXiv:arXiv:astro-ph/9511106. doi:10.1103/PhysRevLett.76.352

[119] C. L. Fryer, Neutron Star Kicks from Asymmetric Collapse, Astrophys. J. Lett. 601 (2004) L175-L178. arXiv:arXiv:astro-ph/0312265 doi:10.1086/382044

[120] E. Müller, M. Rampp, R. Buras, H.-T. Janka, D. H. Shoemaker, Toward Gravitational Wave Signals from Realistic Core-Collapse Supernova Models, Astrophys. J. 603 (2004) 221-230. arXiv:arXiv: astro-ph/0309833 doi:10.1086/381360

[121] M. Rampp, E. Mueller, M. Ruffert, Simulations of non-axisymmetric rotational core collapse, Astron. Astrophys.332 (1998) 969-983. arXiv: arXiv : astro-ph/9711122

[122] C. D. Ott, H. Dimmelmeier, A. Marek, H.-T. Janka, I. Hawke, B. Zink, E. Schnetter, 3D Collapse of Rotating Stellar Iron Cores in General Relativity Including Deleptonization and a Nuclear Equation of State, Physical Review Letters 98 (26) (2007) 261101-+. arXiv:arXiv:astro-ph/0609819. doi:10.1103/PhysRevLett.98.261101

[123] C. D. Ott, A. Burrows, L. Dessart, E. Livne, A New Mechanism for Gravitational-Wave Emission in CoreCollapse Supernovae, Physical Review Letters 96 (20) (2006) 201102-+. arXiv:arXiv:astro-ph/0605493. doi:10.1103/PhysRevLett.96.201102

[124] N. Andersson, V. Ferrari, D. I. Jones, K. D. Kokkotas, B. Krishnan, J. S. Read, L. Rezzolla, B. Zink, Gravitational waves from neutron stars: promises and challenges, General Relativity and Gravitation (2010) 156+ arXiv:0912.0384 doi:10.1007/s10714-010-1059-4

[125] K. Kotake, N. Ohnishi, S. Yamada, Gravitational Radiation from Standing Accretion Shock Instability in Core-Collapse Supernovae, Astrophys. J. 655 (2007) 406-415. arXiv:arXiv:astro-ph/0607224. doi: $10.1086 / 509320$

[126] K. Kotake, W. Iwakami, N. Ohnishi, S. Yamada, Ray-Tracing Analysis of Anisotropic Neutrino Radiation for Estimating Gravitational Waves in Core-Collapse Supernovae, Astrophys. J. 704 (2009) 951-963. arXiv:0909.3622 doi:10.1088/0004-637X/704/2/951

[127] K. Kotake, W. Iwakami, N. Ohnishi, S. Yamada, Stochastic Nature of Gravitational Waves from Supernova Explosions with Standing Accretion Shock Instability, Astrophys. J. Lett. 697 (2009) L133-L136. arXiv:0904.4300 doi: 10.1088/0004-637X/697/2/L133

[128] A. Marek, H.-T. Janka, E. Müller, Equation-of-state dependent features in shock-oscillation modulated neutrino and gravitational-wave signals from supernovae, Astron. Astrophys. 496 (2009) 475-494. arXiv:0808.4136. doi:10.1051/0004-6361/200810883

[129] J. W. Murphy, C. D. Ott, A. Burrows, A Model for Gravitational Wave Emission from Neutrino-Driven CoreCollapse Supernovae, ArXiv e-prints $\operatorname{arXiv:0907.4762}$

[130] W. D. Arnett, C. Meakin, Toward Realistic Progenitors of Core-collapse Supernovae, Astrophys. J.733 (2011) 78-+. arXiv: 1101.5646. doi:10.1088/0004-637X/733/2/78

[131] E. Müller, H. . Janka, A. Wongwathanarat, Parametrized 3D models of neutrino-driven supernova explosions: Neutrino emission asymmetries and gravitational-wave signals, ArXiv e-prints arXiv:1106.6301

[132] R. Epstein, The generation of gravitational radiation by escaping supernova neutrinos, Astrophys. J.223 (1978) 1037-1045. doi:10.1086/156337

[133] M. S. Turner, R. V. Wagoner, Gravitational radiation from slowly-rotating 'supernovae' - Preliminary results, in: L. L. Smarr (Ed.), Sources of Gravitational Radiation, 1979, pp. 383-407.

[134] M. Favata, The gravitational-wave memory effect, Classical and Quantum Gravity 27 (8) (2010) 084036-+. arXiv:1003.3486 doi:10.1088/0264-9381/27/8/084036

[135] T. Hiramatsu, K. Kotake, H. Kudoh, A. Taruya, Gravitational wave background from neutrino-driven gamma-ray bursts, Mon. Not. R. Astron. Soc.364 (2005) 1063-1068. arXiv:arXiv:astro-ph/0509787 
doi:10.1111/j.1365-2966.2005.09643.x

[136] Y. Suwa, K. Murase, Probing the central engine of long gamma-ray bursts and hypernovae with gravitational waves and neutrinos, Phys. Rev. D80 (12) (2009) 123008-+. arXiv:0906.3833 doi:10.1103/PhysRevD.80.123008

[137] Y. Suwa, T. Takiwaki, K. Kotake, K. Sato, Gravitational Wave Background from Population III Stars, Astrophys. J. Lett. 665 (2007) L43-L46. arXiv:arXiv:0706.3495 doi:10.1086/521078

[138] K. Kotake, S. Yamada, K. Sato, Anisotropic Neutrino Radiation in Rotational Core Collapse, Astrophys. J. 595 (2003) 304-316. doi:10.1086/377196

[139] H. Janka, R. Mönchmeyer, Anisotropic neutrino emission from rotating protoneutron stars, Astron. Astrophys.209 (1989) L5-L8.

[140] R. Walder, A. Burrows, C. D. Ott, E. Livne, I. Lichtenstadt, M. Jarrah, Anisotropies in the Neutrino Fluxes and Heating Profiles in Two-dimensional, Time-dependent, Multigroup Radiation Hydrodynamics Simulations of Rotating Core-Collapse Supernovae, Astrophys. J.626 (2005) 317-332. arXiv: arXiv : astro-ph/0412187 doi: $10.1086 / 429816$

[141] L. Dessart, A. Burrows, E. Livne, C. D. Ott, Magnetically Driven Explosions of Rapidly Rotating White Dwarfs Following Accretion-Induced Collapse, Astrophys. J.669 (2007) 585-599. arXiv:0705.3678 doi:10.1086/521701

[142] Y. Suwa, K. Kotake, T. Takiwaki, The Gravitational-wave signatures in a rotating vs. non-rotating $13 \mathrm{Msun}$ star driven by the SASI-aided neutrino-heating supernova mechanism, in preparation.

[143] A. Wongwathanarat, N. J. Hammer, E. Müller, An axis-free overset grid in spherical polar coordinates for simulating 3D self-gravitating flows, Astron. Astrophys.514 (2010) A48+. arXiv:1003.1633. doi:10.1051/0004-6361/200913435

[144] C. L. Fryer, D. E. Holz, S. A. Hughes, Gravitational Waves from Stellar Collapse: Correlations to Explosion Asymmetries, Astrophys. J.609 (2004) 288-300. arXiv: arXiv:astro-ph/0403188 doi:10.1086/421040

[145] K. Kotake, W. Iwakami Nakano, N. Ohnishi, Effects of Rotation on Stochasticity of Gravitational Waves in Nonlinear Phase of Core-Collapse Supernovae, ArXiv e-printsarXiv: 1106.0544

[146] A. Wongwathanarat, H. Janka, E. Mueller, Hydrodynamical Neutron Star Kicks in Three Dimensions, ArXiv e-prints arXiv: 1010.0167

[147] K. S. Thorne, Gravitational Waves, in: E. W. Kolb \& R. D. Peccei (Ed.), Particle and Nuclear Astrophysics and Cosmology in the Next Millenium, 1995, pp. 160-+.

[148] S. e. a. Kawamura, The Japanese space gravitational wave antenna DECIGO, Classical and Quantum Gravity 23 (2006) 125-+. doi:10.1088/0264-9381/23/8/S17

[149] J. M. LeBlanc, J. R. Wilson, A Numerical Example of the Collapse of a Rotating Magnetized Star, Astrophys. J. 161 (1970) 541-+.

[150] G. S. Bisnovatyi-Kogan, I. P. Popov, A. A. Samokhin, The magnetohydrodynamic rotational model of supernova explosion, Astrophys. Sp. Science41 (1976) 287-320. doi:10.1007/BF00646184

[151] E. Müller, W. Hillebrandt, A magnetohydrodynamical supernova model, Astron. Astrophys.80 (1979) $147-154$.

[152] E. M. D. Symbalisty, Magnetorotational iron core collapse, Astrophys. J. 285 (1984) 729-746. doi:10.1086/162551

[153] N. V. Ardeljan, G. S. Bisnovatyi-Kogan, S. G. Moiseenko, Nonstationary magnetorotational processes in a rotating magnetized cloud, Astron. Astrophys. 355 (2000) 1181-1190. arXiv:arXiv:astro-ph/9912513

[154] S. Yamada, H. Sawai, Numerical Study on the Rotational Collapse of Strongly Magnetized Cores of Massive Stars, Astrophys. J. 608 (2004) 907-924. doi:10.1086/420760

[155] K. Kotake, H. Sawai, S. Yamada, K. Sato, Magnetorotational Effects on Anisotropic Neutrino Emission and Convection in Core-Collapse Supernovae, Astrophys. J. 608 (2004) 391-404. doi:10.1086/392530

[156] K. Kotake, S. Yamada, K. Sato, North-South Neutrino Heating Asymmetry in Strongly Magnetized and Rotating Stellar Cores, Astrophys. J.618 (2005) 474-484. arXiv: arXiv:astro-ph/0409244 doi:10.1086/425911

[157] H. Sawai, K. Kotake, S. Yamada, Core-Collapse Supernovae with Nonuniform Magnetic Fields, Astrophys. J. 631 (2005) 446-455. doi:10.1086/432529

[158] M. Obergaulinger, M. A. Aloy, H. Dimmelmeier, E. Müller, Axisymmetric simulations of magnetorotational core collapse: approximate inclusion of general relativistic effects, Astron. Astrophys. 457 (2006) $209-222$. arXiv:arXiv:astro-ph/0602187 doi:10.1051/0004-6361:20064982

[159] M. Obergaulinger, M. A. Aloy, E. Müller, Axisymmetric simulations of magneto-rotational core collapse: dynamics and gravitational wave signal, Astron. Astrophys. 450 (2006) 1107-1134 arXiv:arXiv:astro-ph/0510184 doi:10.1051/0004-6361:20054306

[160] A. Burrows, L. Dessart, E. Livne, C. D. Ott, J. Murphy, Simulations of Magnetically Driven Supernova and Hypernova Explosions in the Context of Rapid Rotation, Astrophys. J. 664 (2007) 416-434. arXiv:arXiv:astro-ph/0702539 doi:10.1086/519161

[161] P. Cerdá-Durán, J. A. Font, H. Dimmelmeier, General relativistic simulations of magneto-rotational core collapse 
with microphysics, ArXiv Astrophysics e-printsarXiv:astro-ph/0703597

[162] Y. Suwa, T. Takiwaki, K. Kotake, K. Sato, Magnetorotational Collapse of Population III Stars, Publ. Astron. Soc. Japan59 (2007) 771-785.

[163] T. Takiwaki, K. Kotake, S. Nagataki, K. Sato, Magneto-driven Shock Waves in Core-Collapse Supernovae, Astrophys. J.616 (2004) 1086-1094. arXiv:arXiv:astro-ph/0408388 doi:10.1086/424993

[164] T. Takiwaki, K. Kotake, K. Sato, Special Relativistic Simulations of Magnetically Dominated Jets in Collapsing Massive Stars, Astrophys. J. 691 (2009) 1360-1379. arXiv:0712.1949. doi: 10.1088/0004-637X/691/2/1360

[165] S. Scheidegger, R. Kaeppeli, S. C. Whitehouse, T. Fischer, M. Liebendoerfer, The influence of model parameters on the prediction of gravitational wave signals from stellar core collapse, ArXiv e-printsarXiv:1001.1570

[166] M. Obergaulinger, H.-T. Janka, Magnetic field amplification in collapsing, non-rotating stellar cores, ArXiv eprintsarXiv:1101.1198

[167] R. C. Duncan, C. Thompson, Formation of very strongly magnetized neutron stars - Implications for gamma-ray bursts, Astrophys. J. Lett. 392 (1992) L9-L13. doi:10.1086/186413

[168] J. M. Lattimer, M. Prakash, Neutron star observations: Prognosis for equation of state constraints, Phys. Rep.442 (2007) 109-165. arXiv:arXiv:astro-ph/0612440 doi:10.1016/j.physrep.2007.02.003

[169] D. L. Meier, R. I. Epstein, W. D. Arnett, D. N. Schramm, Magnetohydrodynamic phenomena in collapsing stellar cores, Astrophys. J. 204 (1976) 869-878.

[170] S. A. Balbus, J. F. Hawley, Instability, turbulence, and enhanced transport in accretion disks, Reviews of Modern Physics 70 (1998) 1-53.

[171] M. Obergaulinger, P. Cerdá-Durán, E. Müller, M. A. Aloy, Semi-global simulations of the magnetorotational instability in core collapse supernovae, Astron. Astrophys.498 (2009) 241-271. arXiv:0811.1652. doi:10.1051/0004-6361/200811323

[172] S. E. Woosley, J. S. Bloom, The Supernova Gamma-Ray Burst Connection, Annual Rev. Astron. Astrophys.44 (2006) 507-556. arXiv:arXiv : astro-ph/0609142 doi:10.1146/annurev.astro.43.072103.150558

[173] M. Shibata, Y. T. Liu, S. L. Shapiro, B. C. Stephens, Magnetorotational collapse of massive stellar cores to neutron stars: Simulations in full general relativity, Phys. Rev. D74 (10) (2006) 104026-+. arXiv:arXiv:astro-ph/0610840 doi:10.1103/PhysRevD.74.104026

[174] T. Takiwaki, K. Kotake, Gravitational-Wave Signatures in Magnetically-driven Supernova Explosions, ArXiv eprints, ApJ in pressarXiv: 1004.2896

[175] K. S. Thorne, Multipole expansions of gravitational radiation, Reviews of Modern Physics 52 (1980) 299-340. doi:10.1103/RevModPhys.52.299

[176] H. Kudoh, A. Taruya, T. Hiramatsu, Y. Himemoto, Detecting a gravitational-wave background with nextgeneration space interferometers, Phys. Rev. D73 (6) (2006) 064006-+. arXiv:arXiv:gr-qc/0511145 doi:10.1103/PhysRevD.73.064006

[177] S. Chandrasekhar, Ellipsoidal figures of equilibrium, 1969.

[178] J.-L. Tassoul, Theory of rotating stars, Princeton Series in Astrophysics, Princeton: University Press, $1978,1978$.

[179] M. Rampp, E. Mueller, M. Ruffert, Simulations of non-axisymmetric rotational core collapse, Astron. Astrophys. 332 (1998) 969-983.

[180] T. Zwerger, E. Mueller, Dynamics and gravitational wave signature of axisymmetric rotational core collapse., Astron. Astrophys. 320 (1997) 209-227.

[181] C. L. Fryer, M. S. Warren, The Collapse of Rotating Massive Stars in Three Dimensions, Astrophys. J.601 (2004) 391-404. arXiv:arXiv:astro-ph/0309539 doi:10.1086/380193

[182] M. Shibata, Y.-I. Sekiguchi, Three-dimensional simulations of stellar core collapse in full general relativity: Nonaxisymmetric dynamical instabilities, Phys. Rev. D71 (2) (2005) 024014. arXiv:arXiv:astro-ph/0412243. doi:10.1103/PhysRevD.71.024014

[183] C. D. Ott, H. Dimmelmeier, A. Marek, H.-T. Janka, B. Zink, I. Hawke, E. Schnetter, Rotating collapse of stellar iron cores in general relativity, Classical and Quantum Gravity 24 (2007) 139-+. arXiv:arXiv:astro-ph/0612638 doi:10.1088/0264-9381/24/12/S10

[184] D. Lai, S. L. Shapiro, Gravitational radiation from rapidly rotating nascent neutron stars, Astrophys. J.442 (1995) 259-272. arXiv:arXiv:astro-ph/9408053 doi:10.1086/175438

[185] K. D. Kokkotas, Gravitational Wave Astronomy, ArXiv e-print $\operatorname{arXiv:0809.1602}$

[186] C. D. Ott, S. Ou, J. E. Tohline, A. Burrows, One-armed Spiral Instability in a Low-T/-W- Postbounce Supernova Core, Astrophys. J. Lett.625 (2005) L119-L122. arXiv:arXiv:astro-ph/0503187 doi:10.1086/431305

[187] M. Shibata, S. Karino, Y. Eriguchi, Dynamical instability of differentially rotating stars, Mon. Not. R. Astron. Soc.334 (2002) L27-L31. arXiv:arXiv: gr-qc/0206002 doi:10.1046/j.1365-8711.2002.05724.x

[188] M. Saijo, T. W. Baumgarte, S. L. Shapiro, One-armed Spiral Instability in Differentially Rotating Stars, Astrophys J.595 (2003) 352-364. arXiv: arXiv: astro-ph/0302436 doi:10.1086/377334 
[189] A. L. Watts, N. Andersson, D. I. Jones, The Nature of Low T/-W- Dynamical Instabilities in Differentially Rotating Stars, Astrophys. J. Lett.618 (2005) L37-L40. arXiv:arXiv:astro-ph/0309554. doi:10.1086/427653

[190] S. Ou, J. E. Tohline, Unexpected Dynamical Instabilities in Differentially Rotating Neutron Stars, Astrophys. J.651 (2006) 1068-1078. arXiv:arXiv:astro-ph/0604099 doi:10.1086/507597

[191] P. Cerdá-Durán, V. Quilis, J. A. Font, AMR simulations of the low T/-W- bar-mode instability of neutron stars, Computer Physics Communications 177 (2007) 288-297. arXiv:0704.0356 doi:10.1016/j.cpc.2007.04.001

[192] M. Saijo, S. Yoshida, Low T/—W-dynamical instability in differentially rotating stars: diagnosis with canonical angular momentum, Mon. Not. R. Astron. Soc.368 (2006) 1429-1442. arXiv:arXiv:astro-ph/0505543. doi:10.1111/j.1365-2966.2006.10229.x

[193] J. C. B. Papaloizou, J. E. Pringle, The dynamical stability of differentially rotating discs. II, Mon. Not. R. Astron. Soc.213 (1985) 799-820.

[194] J. M. Centrella, K. C. B. New, L. L. Lowe, J. D. Brown, Dynamical Rotational Instability at Low T/W, Astrophys J. Lett.550 (2001) L193-L196. arXiv: arXiv:astro-ph/0010574 doi:10.1086/319634

[195] T. Lund, A. Marek, C. Lunardini, H.-T. Janka, G. Raffelt, Fast time variations of supernova neutrino fluxes and their detectability, Phys. Rev. D82 (6) (2010) 063007-+. arXiv:1006.1889. doi:10.1103/PhysRevD.82.063007

[196] I. Leonor, L. Cadonati, E. Coccia, S. D’Antonio, A. Di Credico, V. Fafone, R. Frey, W. Fulgione, E. Katsavounidis, C. D. Ott, G. Pagliaroli, K. Scholberg, E. Thrane, F. Vissani, Searching for prompt signatures of nearby core-collapse supernovae by a joint analysis of neutrino and gravitational-wave data, ArXiv eprintsarXiv:1002.1511

[197] S. Nishimura, K. Kotake, M.-a. Hashimoto, S. Yamada, N. Nishimura, S. Fujimoto, K. Sato, r-Process Nucleosynthesis in Magnetohydrodynamic Jet Explosions of Core-Collapse Supernovae, Astrophys. J.642 (2006) 410-419. arXiv:arXiv:astro-ph/0504100 doi:10.1086/500786

[198] S.-i. Fujimoto, M.-a. Hashimoto, K. Kotake, S. Yamada, Heavy-Element Nucleosynthesis in a Collapsar, Astrophys. J.656 (2007) 382-392. arXiv: arXiv : astro-ph/0602460 doi:10.1086/509908

[199] S. Wanajo, H.-T. Janka, S. Kubono, Uncertainties in the $v$ p-process: Supernova Dynamics Versus Nuclear Physics, Astrophys. J.729 (2011) 46-+. arXiv:1004.4487 doi:10.1088/0004-637X/729/1/46

[200] S. Wanajo, H.-T. Janka, The r-process in the neutrino-driven wind from a black-hole torus, ArXiv eprintsarXiv:1106.6142

[201] J. M. Lattimer, F. D. Swesty, A generalized equation of state for hot, dense matter, Nuclear Physics A 535 (1991) 331-376. doi:10.1016/0375-9474(91)90452-C

[202] S. Shlomo, V. M. Kolomietz, G. Colò, Deducing the nuclear-matter incompressibility coefficient from data on isoscalar compression modes, European Physical Journal A 30 (2006) 23-30. doi:10.1140/epja/i2006-10100-3

[203] P. B. Demorest, T. Pennucci, S. M. Ransom, M. S. E. Roberts, J. W. T. Hessels, A two-solar-mass neutron star measured using Shapiro delay, Nature467 (2010) 1081-1083. arXiv:1010.5788 doi:10.1038/nature09466

[204] E. O'Connor, C. D. Ott, Black Hole Formation in Failing Core-Collapse Supernovae, Astrophys. J.730 (2011) 70-+. arXiv: 1010.5550 doi:10.1088/0004-637X/730/2/70

[205] K. Kiuchi, K. Kotake, Equilibrium configurations of strongly magnetized neutron stars with realistic equations of state, Mon. Not. R. Astron. Soc.385 (2008) 1327-1347. arXiv:0708.3597 doi:10.1111/j.1365-2966.2007.12791.x

[206] T. Fischer, I. Sagert, G. Pagliara, M. Hempel, J. Schaffner-Bielich, T. Rauscher, F.-K. Thielemann, R. Käppeli, G. Martínez-Pinedo, M. Liebendörfer, Core-collapse Supernova Explosions Triggered by a Quark-Hadron Phase Transition During the Early Post-bounce Phase, Astrophys. J. Supp.194 (2011) 39-+. arXiv:1011.3409. doi:10.1088/0067-0049/194/2/39

[207] T. A. Thompson, E. Quataert, A. Burrows, Viscosity and Rotation in Core-Collapse Supernovae, Astrophys. J.620 (2005) 861-877. arXiv:arXiv:astro-ph/0403224 doi:10.1086/427177

[208] Y. Masada, T. Takiwaki, K. Kotake, submitted to Astrophys. J..

[209] T. K. Suzuki, K. Sumiyoshi, S. Yamada, Alfvén Wave-Driven Supernova Explosion, Astrophys. J.678 (2008) 1200-1206. arXiv:0707.4345 doi:10.1086/533515

[210] J. Guilet, J. Sato, T. Foglizzo, The Saturation of SASI by Parasitic Instabilities, Astrophys. J.713 (2010) 1350 1362. arXiv:0910.3953 doi:10.1088/0004-637X/713/2/1350

[211] J. W. Murphy, C. Meakin, A Global Turbulence Model for Neutrino-Driven Convection in Core-Collapse Supernovae, ArXiv e-printsarXiv:1106.5496

[212] C. D. Ott, C. Reisswig, E. Schnetter, E. O’Connor, U. Sperhake, F. Löffler, P. Diener, E. Abdikamalov, I. Hawke, A. Burrows, Dynamics and Gravitational Wave Signature of Collapsar Formation, Physical Review Letters 106 (16) (2011) 161103-+. arXiv:1012.1853 doi:10.1103/PhysRevLett.106.161103 
[213] K. Sumiyoshi, S. Yamada, H. Suzuki, S. Chiba, Neutrino Signals from the Formation of a Black Hole: A Probe of the Equation of State of Dense Matter, Physical Review Letters 97 (9) (2006) 091101-+. arXiv:arXiv:astro-ph/0608509 doi:10.1103/PhysRevLett.97.091101

[214] K. Nakazato, K. Sumiyoshi, H. Suzuki, S. Yamada, Exploring hadron physics in black hole formations: A new promising target of neutrino astronomy, Phys. Rev. D81 (8) (2010) 083009-+. arXiv:1004.0291. doi:10.1103/PhysRevD.81.083009

[215] K. Nakazato, K. Sumiyoshi, S. Yamada, Impact of Quarks and Pions on Dynamics and Neutrino Signal of Black Hole Formation in Non-rotating Stellar Core Collapse, Astrophys. J.721 (2010) 1284-1294. arXiv:1001.5084. doi:10.1088/0004-637X/721/2/1284

[216] K. Fukushima, T. Hatsuda, The phase diagram of dense QCD, Reports on Progress in Physics 74 (1) (2011) 014001-+. arXiv:1005.4814 doi:10.1088/0034-4885/74/1/014001

[217] B. Paczynski, Super-Eddington winds from neutron stars, Astrophys. J.363 (1990) 218-226. doi:10.1086/169332

[218] P. Meszaros, M. J. Rees, High-entropy fireballs and jets in gamma-ray burst sources, Mon. Not. R. Astron. Soc.257 (1992) 29P-31P.

[219] T. A. Thompson, P. Chang, E. Quataert, Magnetar Spin-Down, Hyperenergetic Supernovae, and Gamma-Ray Bursts, Astrophys. J. 611 (2004) 380-393. arXiv:arXiv:astro-ph/0401555 doi:10.1086/421969

[220] D. A. Uzdensky, A. I. MacFadyen, Magnetar-Driven Magnetic Tower as a Model for Gamma-Ray Bursts and Asymmetric Supernovae, Astrophys. J. 669 (2007) 546-560. arXiv:arXiv:astro-ph/0609047. doi: $10.1086 / 521322$

[221] S. E. Woosley, Gamma-ray bursts from stellar mass accretion disks around black holes, Astrophys. J.405 (1993) 273-277. doi:10.1086/172359

[222] B. Paczynski, Are Gamma-Ray Bursts in Star-Forming Regions?, Astrophys. J. Lett.494 (1998) L45+. arXiv:arXiv:astro-ph/9710086 doi:10.1086/311148

[223] A. I. MacFadyen, S. E. Woosley, Collapsars: Gamma-Ray Bursts and Explosions in "Failed Supernovae", Astrophys. J. 524 (1999) 262-289. arXiv:arXiv:astro-ph/9810274 doi:10.1086/307790

[224] Z. G. Dai, T. Lu, Gamma-ray burst afterglows and evolution of postburst fireballs with energy injection from strongly magnetic millisecond pulsars, Astron. Astrophys.333 (1998) L87-L90. arXiv:arXiv:astro-ph/9810402

[225] N. Bucciantini, E. Quataert, J. Arons, B. D. Metzger, T. A. Thompson, Relativistic jets and long-duration gammaray bursts from the birth of magnetars, Mon. Not. R. Astron. Soc.(2007) L126+arXiv:arXiv:0707.2100 doi:10.1111/j.1745-3933.2007.00403.x

[226] B. D. Metzger, D. Giannios, T. A. Thompson, N. Bucciantini, E. Quataert, The protomagnetar model for gamma-ray bursts, Mon. Not. R. Astron. Soc.413 (2011) 2031-2056. arXiv:1012.0001. doi:10.1111/j.1365-2966.2011.18280.x

[227] S. Harikae, T. Takiwaki, K. Kotake, Long-Term Evolution of Slowly Rotating Collapsar in Special Relativistic Magnetohydrodynamics, Astrophys. J. 704 (2009) 354-371. arXiv:0905.2006. doi:10.1088/0004-637X/704/1/354

[228] S. Harikae, K. Kotake, T. Takiwaki, Neutrino Pair Annihilation in Collapsars: A Ray-tracing Method in Special Relativity, Astrophys. J. 713 (2010) 304-317. arXiv:0912.2590 doi:10.1088/0004-637X/713/1/304

[229] S. E. Woosley, A. Heger, Nucleosynthesis and remnants in massive stars of solar metallicity, Phys. Rep.442 (2007) 269-283. arXiv:arXiv:astro-ph/0702176 doi:10.1016/j.physrep.2007.02.009

[230] F.-K. Thielemann, R. Hirschi, M. Liebendörfer, R. Diehl, Massive Stars and Their Supernovae, in: R. Diehl, D. H. Hartmann, \& N. Prantzos (Ed.), Lecture Notes in Physics, Berlin Springer Verlag, Vol. 812 of Lecture Notes in Physics, Berlin Springer Verlag, 2011, pp. 153-232. arXiv:1008.2144 